\title{
THE DISTANCES TO OPEN CLUSTERS FROM MAIN-SEQUENCE FITTING. III. IMPROVED ACCURACY WITH EMPIRICALLY CALIBRATED ISOCHRONES
}

\author{
Deokkeun An, ${ }^{1}$ Donald M. Terndrup, ${ }^{1}$ Marc H. Pinsonneault, ${ }^{1}$ Diane B. Paulson, ${ }^{2}$ \\ Robert B. Hanson, ${ }^{3}$ and John R. Stauffer ${ }^{4}$ \\ Received 2006 July 20; accepted 2006 September 21
}

\begin{abstract}
We continue our series of papers on open cluster distances with a critical assessment of the accuracy of mainsequence fitting using isochrones that employ empirical corrections to the color-temperature relations. We use four nearby open clusters with multicolor photometry and accurate metallicities and present a new metallicity for Praesepe $([\mathrm{Fe} / \mathrm{H}]=+0.11 \pm 0.03)$ from high-resolution spectra. The internal precision of distance estimates is about a factor of 5 better than the case without the color calibrations. After taking into account all major systematic errors, we obtain distances accurate to about $2 \%-3 \%$ when there exists a good metallicity estimate. Metallicities accurate to better than 0.1 dex may be obtained from $B V I_{\mathrm{C}} K_{s}$ photometry alone. We also derive a helium abundance for the Pleiades of $Y=0.279 \pm 0.015$, which is equal within the errors to the Sun's initial helium abundance and that of the Hyades. Our best estimates of distances are $(m-M)_{0}=6.33 \pm 0.04,8.03 \pm 0.04$, and $9.61 \pm 0.03$ to Praesepe, NGC 2516, and M67, respectively. Our Pleiades distance at the spectroscopic metallicity, $(m-M)_{0}=5.66 \pm 0.01$ (internal) \pm 0.05 (systematic), is in excellent agreement with several geometric distance measurements. We have made calibrated isochrones for $-0.3 \leq[\mathrm{Fe} / \mathrm{H}] \leq+0.2$ available online.
\end{abstract}

Subject headings: Hertzsprung-Russell diagram — open clusters and associations: individual (M67, NGC 2516, Pleiades, Praesepe) — stars: abundances — stars: activity — stars: distances —

stars: evolution

\section{INTRODUCTION}

The determination of accurate distances is the key to understanding how stars and the Galaxy have formed and evolved. From protostars in star-forming regions to ancient tracers of the halo, improved distances have refined stellar evolutionary theory and Galactic structure models (e.g., Reid 1999). The Hipparcos mission (Perryman et al. 1997b) was especially valuable, providing trigonometric parallaxes for $\sim 10^{5}$ stars to precision of 1-2 mas (Perryman et al. 1997a). These parallaxes, however, are only useful for individual stars within $\sim 100 \mathrm{pc}$. Most open clusters are much more distant than this "horizon," but a half-dozen of the nearest clusters have 10-50 or more Hipparcos stars, yielding cluster parallaxes ostensibly accurate to $5 \%$ or better (Mermilliod et al. 1997; Perryman et al. 1998; Robichon et al. 1999; van Leeuwen 1999).

Main-sequence (MS) fitting, also known as the photometric parallax method (e.g., Johnson 1957; Siegel et al. 2002), has long been used to estimate distances to individual stars and star clusters beyond the limits of parallax studies and is considered to be a robust and well-understood technique. It was therefore a big surprise when the Hipparcos distances to the Pleiades and Coma Ber open clusters were in disagreement with distances from the MS fitting at more than a $3 \sigma$ level (Pinsonneault et al. 1998). It is difficult to reconcile a short Pleiades distance with stellar interior and spectroscopic abundance studies. A high helium abundance

\footnotetext{
1 Department of Astronomy, Ohio State University, Columbus, OH; deokkeun@ astronomy.ohio-state.edu, terndrup@astronomy.ohio-state.edu,pinsono@astronomy ohio-state.edu.

2 Planetary Systems Branch, NASA Goddard Space Flight Center, Greenbelt, MD; diane.b.paulson@gsfc.nasa.gov.

${ }^{3}$ University of California Observatories/Lick Observatory, Santa Cruz, CA; hanson@ucolick.org.

4 Infrared Processing and Analysis Center, California Institute of Technology, Pasadena, CA; stauffer@ipac.caltech.edu.
}

would make a cluster fainter than expected from its metallicity, and solutions of this type have been discussed in the literature for the Pleiades (Belikov et al. 1998). However, this is difficult to understand since there do not seem to be nearby field stars of similar characteristics in the Hipparcos catalog (Soderblom et al. 1998), and the helium enhancement would have to be enormous $(Y \approx 0.34)$. In addition, it has been suggested that the metal abundance from spectroscopy may have been significantly overestimated (Percival et al. 2003). An argument was also made that distance estimates from theoretical stellar models have been overestimated for young clusters due to unknown, age-related physics (van Leeuwen 1999).

However, the most likely explanation is related to the Hipparcos parallaxes themselves. Pinsonneault et al. (1998) showed that the 12 bright stars near the center of the Pleiades all had virtually the same parallax, $\sim 9$ mas, more than 1 mas larger than the mean parallax for other cluster stars. They attributed this to a local zero-point error of the individual stellar parallaxes that are correlated over the Hipparcos $0.9^{\circ}$ field of view (van Leeuwen $\&$ Evans 1998). These quasi-random errors were caused by the Hipparcos great circle data reductions, as Makarov $(2002,2003)$ proved by rereducing the Pleiades and Comar Ber cluster parallaxes in a different way that correctly obtains the absolute zero point of parallax. Additional effects may result from the way the Hipparcos data were obtained and analyzed, and a more elaborate reduction of the Hipparcos parallaxes promises to produce improved distances and better understood errors (van Leeuwen 2005; van Leeuwen \& Fantino 2005).

The discrepant Hipparcos result for the Pleiades subsequently led to many efforts to determine the cluster's distance from binaries and independent parallax measurements (e.g., Munari et al. 2004; Pan et al. 2004; Johns-Krull \& Anderson 2005; Soderblom et al. 2005). These results support the longer distance scale from MS fitting, verifying that the Hipparcos result was in error. With a formal error of $\sim 1 \%$ from these measurements, the Pleiades 
represents a second system (besides the Hyades) with a sufficiently accurate distance for a precision test of stellar evolutionary models.

Even though the controversy over the Pleiades distance is now settled, a critical assessment of the MS fitting technique is still required to reliably estimate a distance. In fact, MS fitting using theoretical isochrones is a complex process that involves both physical and empirical considerations (e.g., Stauffer 2001). There are, however, many opportunities to check the construction of the isochrones. Stellar evolution models can be tested against the Sun and other stars, such as eclipsing binaries, that have accurate masses and radii. Furthermore, multicolor photometry in nearby clusters and field stars can be used to test the bolometric corrections and color-effective temperature $\left(T_{\text {eff }}\right)$ relations to transform theoretical quantities (luminosity and $T_{\text {eff }}$ ) to magnitudes and colors (e.g., VandenBerg \& Clem 2003).

In our first two papers of this series (Pinsonneault et al. 2003, 2004, hereafter Paper I and Paper II, respectively) we began a long-term effort to assess the accuracy of distances from MS fitting and to reduce or eliminate systematic errors in the process, particularly those arising from the transformation of theoretical to observational quantities. In Paper I we demonstrated that stellar models from the Yale Rotating Evolutionary Code (YREC; Sills et al. 2000) are in good agreement with masses and luminosities for the well-studied Hyades eclipsing binary vB 22 (Torres \& Ribas 2002). These models also satisfy stringent tests from helioseismology and predict solar neutrino fluxes in line with observations (Basu et al. 2000; Bahcall et al. 2001; Bahcall $\&$ Pinsonneault 2004). In Paper II we showed that the models provide a good match to the spectroscopically determined temperatures (Paulson et al. 2003) for individual Hyades members with good parallaxes (de Bruijne et al. 2001). However, we found that any of the widely used color- $T_{\text {eff }}$ relations (e.g., Alonso et al. 1995, 1996; Lejeune et al. 1997, 1998) fail to reproduce the observed shapes of the MS in the Hyades; differences in broadband colors were as large as $\sim 0.1 \mathrm{mag}$. The existence of these systematic errors in the colors in the presence of agreement between the spectroscopic and theoretical $L-T_{\text {eff }}$ scales strongly implies that there are problems with the adopted color- $T_{\text {eff }}$ relations instead of errors in the theoretical $T_{\text {eff }}$ scale. Therefore, we proposed empirical corrections to the color- $T_{\text {eff }}$ relations from Lejeune et al. $(1997,1998)$ that were adopted in the isochrone computations.

In this study we generate a set of isochrones over a wide range of age and metallicity and test the validity of the Hyades-based color- $T_{\text {eff }}$ corrections using extensive multicolor photometry of four well-studied nearby open clusters. We show that isochrones employing the Hyades empirical corrections precisely match the observed MS shapes, except where anomalously blue colors in young open clusters have been previously noted (Stauffer et al. 2003). Furthermore, we demonstrate that the empirical corrections improve the internal precision of the isochrones by examining the consistency of distances derived from several color-magnitude diagrams (CMDs).

We also assess various sources of systematic errors in the MS fitting technique. Previously, Pinsonneault et al. (1998) considered the effects of age, metal abundance, helium, reddening, and systematic errors in the photometry, demonstrating that these could not explain the short distance to the Pleiades from Hipparcos. Terndrup et al. (2002) paid attention to the adopted reddening law in a discussion of the distance to NGC 2516. Here we extend the error analysis more quantitatively, emphasizing photometric calibration issues and the bias in distance estimates induced by the presence of unresolved cluster binaries or field foreground/background stars.
This paper also explores the effect of metallicity on the luminosity of the MS. Metallicity changes isochrone luminosities more strongly than many other input parameters, and the degree of sensitivity depends on the color index used. This permits a purely photometric derivation of the metallicity (e.g., Pinsonneault et al. 1998, 2000; Stello \& Nissen 2001; Terndrup et al. 2002), which can be compared to metallicities derived from high-resolution spectra. An agreement between the photometric and spectroscopic metallicities, as we find in this paper, provides supporting evidence that the effects of metallicity on the theoretical quantities $\left(L, T_{\text {eff }}\right)$ and on the color- $T_{\text {eff }}$ relations are correctly computed.

The distances in this paper are tied to the Hyades distance at $(m-M)_{0}=3.33 \pm 0.01(d=46.34 \pm 0.27 \mathrm{pc})$, the cluster's center of mass inferred from the Hipparcos catalog (Perryman et al. 1998). Unlike the controversial Hipparcos distance to the Pleiades, the large angular diameter of the Hyades on the sky makes the cluster parallax less vulnerable to the spatial correlation of the Hipparcos parallax (Narayanan \& Gould 1999a, 1999b; de Bruijne et al. 2001).

In $\S 2$ we compile cluster photometry, metallicities, reddening estimates, and information on binarity and membership and present a metallicity for Praesepe from new high-resolution spectra. In $\S 3$ we briefly describe the construction of the isochrones. In $\S 4$ we compute the distances to the sample clusters with the reddening fixed at previously known values and demonstrate that the empirical corrections improve the internal precision of the isochrones. In $\S 5$ we simultaneously solve for the cluster metallicity, reddening, and distance from the $\chi^{2}$ minimization. In $\S 6$ we evaluate the effects of several systematic error sources, including those from cluster binaries and field star contamination. In $\S 7$ we discuss several implications of our results. In the Appendix we address issues on the photometric zero points of the empirical Hyades isochrone.

\section{OPEN CLUSTER DATA}

\subsection{Selection of Clusters}

We consider four nearby Galactic open clusters with extensive multicolor photometry: Praesepe (=M44; NGC 2632), the Pleiades (=M45), M67 (=NGC 2682), and NGC 2516. The choice of these clusters was motivated by several factors. All have welldetermined estimates of metal abundance and reddening against which we can compare photometrically derived values. Samples in Praesepe, the Pleiades, and M67 are dominated by known cluster members, so we can examine whether the Hyades-based color calibration from Paper II generates isochrones that precisely match the shapes of the MS in these clusters. Praesepe has extensive information on binarity, so systematic errors in distances arising from the presence of binaries can be explored. M67 and NGC 2516 each have modern photometry from two independent studies, from which we gauge the sizes of errors that arise from photometric calibration issues. In addition, NGC 2516 has a relatively high reddening compared to the other clusters, which allows us to evaluate the consequences of adopting particular reddening laws.

The Pleiades is a special case because its distance has recently been accurately measured from astrometric and eclipsing binary studies and from ground- and space-based parallaxes, allowing a precise test of distances derived from MS fitting. Individual measurements of these studies are summarized in Table 1, and the weighted average distance from these measurements is $(m-M)_{0}=5.632 \pm 0.017$. The Hipparcos distances to the Pleiades and other clusters are discussed in $\S 7.2$. 
TABLE 1

Recent Measurements of the Pleiades Distance

\begin{tabular}{|c|c|c|}
\hline Reference & Method & $(m-M)_{0}$ \\
\hline Narayanan \& Gould (1999b)................ & Moving cluster & $5.58 \pm 0.18$ \\
\hline Gatewood et al. (2000) ........................... & Ground parallax & $5.58 \pm 0.12$ \\
\hline 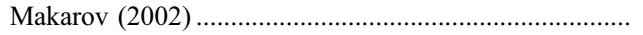 & Hipparcos reanalysis & $5.55 \pm 0.06$ \\
\hline Munari et al. (2004) & Eclipsing binary (HD 23642) & $5.60 \pm 0.03$ \\
\hline 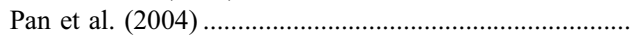 & Astrometric binary (Atlas) & $5.65 \pm 0.03$ \\
\hline Zwahlen et al. (2004) ....................... & Astrometric binary (Atlas) & $5.60 \pm 0.07$ \\
\hline Johns-Krull \& Anderson (2005)... & $H S T$ parallax & $5.66 \pm 0.06$ \\
\hline 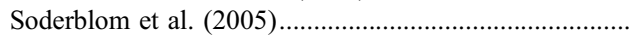 & $H S T$ parallax & $5.65 \pm 0.05$ \\
\hline Southworth et al. (2005) & Eclipsing binary (HD 23642) & $5.72 \pm 0.05$ \\
\hline Weighted mean & $\ldots$ & $5.63 \pm 0.02$ \\
\hline
\end{tabular}

We use cluster ages from Meynet et al. (1993) for the Pleiades (100 Myr), M67 (4 Gyr), and NGC 2516 (140 Myr). Praesepe is generally considered to be the same age as the Hyades (e.g., Mermilliod 1981a); in Paper II, we assumed an age of $550 \mathrm{Myr}$ for the Hyades, as derived from isochrones without overshooting (Perryman et al. 1998). As we demonstrate, the cluster distances are insensitive to the choice of age.

\subsection{Photometry}

\subsubsection{Praesepe and the Pleiades}

We compiled optical photometry for Praesepe and the Pleiades mainly from WEBDA ${ }^{5}$ (Mermilliod \& Paunzen 2003) and the Open Cluster Database. ${ }^{6}$ Data in $V$ and $B-V$ for Praesepe came from Johnson (1952), Dickens et al. (1968), and Castelaz et al. (1991). Following the suggestion by Dickens et al. (1968), we added +0.002 to $V$ and +0.006 to their $B-V$ to match the Johnson (1952) data. We adopted the photometry from Castelaz et al. (1991) without alteration; the average difference in $B-V$ is $+0.002 \pm 0.012$, Johnson (1952) being redder, among 39 stars in common (including nonmembers). We compared the numerous sources of $V$ and $B-V$ in the Pleiades but did not find statistically significant differences in any sample. At $B-V \gtrsim 0.8$, the scatter in measurements from several sources often exceeded the stated photometric errors; this may result from brightness and color changes from stellar spots on rapidly rotating stars in young open clusters (Stauffer et al. 2003). For stars in this color range, as everywhere else, we simply averaged the several available colors and magnitudes.

The situation in $V-I_{\mathrm{C}}$ is less straightforward because data for Praesepe and the Pleiades, like those for the Hyades, came from either the Johnson $\left(V-I_{\mathrm{J}}\right)$ or the Kron $\left(V-I_{\mathrm{K}}\right)$ systems. The

\footnotetext{
${ }^{5}$ See http://obswww.unige.ch/webda/webda.html.

${ }^{6}$ See http://spider.ipac.caltech.edu/staff/stauffer/opencl/index.html.
}

colors on the Johnson system were transformed to the Cousins $\left(V-I_{\mathrm{C}}\right)$ system using an updated transformation equation as described in the Appendix. The Kron colors were transformed to the Cousins system using the cubic polynomial derived by Bessell \& Weis (1987).

In Praesepe, stars with $V<12$ have $V-I$ photometry on the Johnson system from Mendoza (1967) and Castelaz et al. (1991), while fainter stars have photometry on the Kron system from Upgren et al. (1979), Weis (1981), Stauffer (1982c), and Mermilliod et al. (1990). Intercomparisons showed that the Mendoza (1967) and Castelaz et al. (1991) colors were on the same system. The same is true for the Kron data except that the photometry from Upgren et al. (1979) required a redward shift of +0.03 in $V-I_{\mathrm{K}}$ to match the other photometry. In the Pleiades, stars with $V<10$ have $V-I$ photometry on the Johnson system from Johnson et al. (1966), Mendoza (1967), and Iriarte (1969), while fainter stars have photometry on the Kron system from Stauffer (1982a, 1982b, 1984), Stauffer \& Hartmann (1987), Stauffer et al. (1989, 1994), and Prosser et al. (1991). Direct comparison between the Mendoza (1967) photometry and that of Iriarte (1969) showed that they agree well for $V-I_{\mathrm{J}} \leq 0.5$, but the Mendoza (1967) data are systematically redder by 0.02 mag for stars with $V-I_{\mathrm{J}} \geq 0.5$. We opted to adjust the red Mendoza (1967) photometry to place them on the Iriarte (1969) scale.

\subsubsection{M67 and NGC 2516}

For $\mathrm{M} 67$, we used $B V I_{\mathrm{C}}$ photometry from Montgomery et al. (1993, hereafter MMJ93) and also from Sandquist (2004, hereafter S04); these are analyzed separately. S04 provided a comparison between the two, revealing statistically significant differences between the two studies. In Table 2 we compile the mean differences in the photometry for M67 and for other clusters as is discussed below. The first column of the table lists the cluster name, and the sense of the comparison is shown in the second column. The last three columns display the mean difference and its standard

TABLE 2

Differences in the Photometry

\begin{tabular}{|c|c|c|c|c|}
\hline Cluster & Comparison & $\langle\Delta V\rangle$ & $\langle\Delta(B-V)\rangle$ & $\left\langle\Delta(V-I)_{\mathrm{C}}\right\rangle$ \\
\hline M67 ............... & MMJ93 - S04 & $+0.017 \pm 0.002$ & $+0.009 \pm 0.002$ & $+0.022 \pm 0.001$ \\
\hline NGC $2516 \ldots$. & S02 - JTH01 & $+0.016 \pm 0.001$ & $-0.003 \pm 0.001$ & $+0.011 \pm 0.001$ \\
\hline Hyades........... & Ground - Tycho- $1^{\mathrm{a}}$ & $+0.012 \pm 0.002$ & $-0.009 \pm 0.001$ & $\ldots$ \\
\hline Praesepe ..... & Ground - Tycho- $1^{\mathrm{a}}$ & $+0.009 \pm 0.007$ & $+0.004 \pm 0.003$ & $\ldots$ \\
\hline 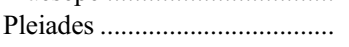 & Ground - Tycho- $1^{\mathrm{a}}$ & $-0.017 \pm 0.003$ & $+0.000 \pm 0.002$ & $\ldots$ \\
\hline rms. & $\ldots$ & 0.015 & 0.006 & 0.017 \\
\hline
\end{tabular}

a Computed for stars with $V_{T} \leq 9$. 


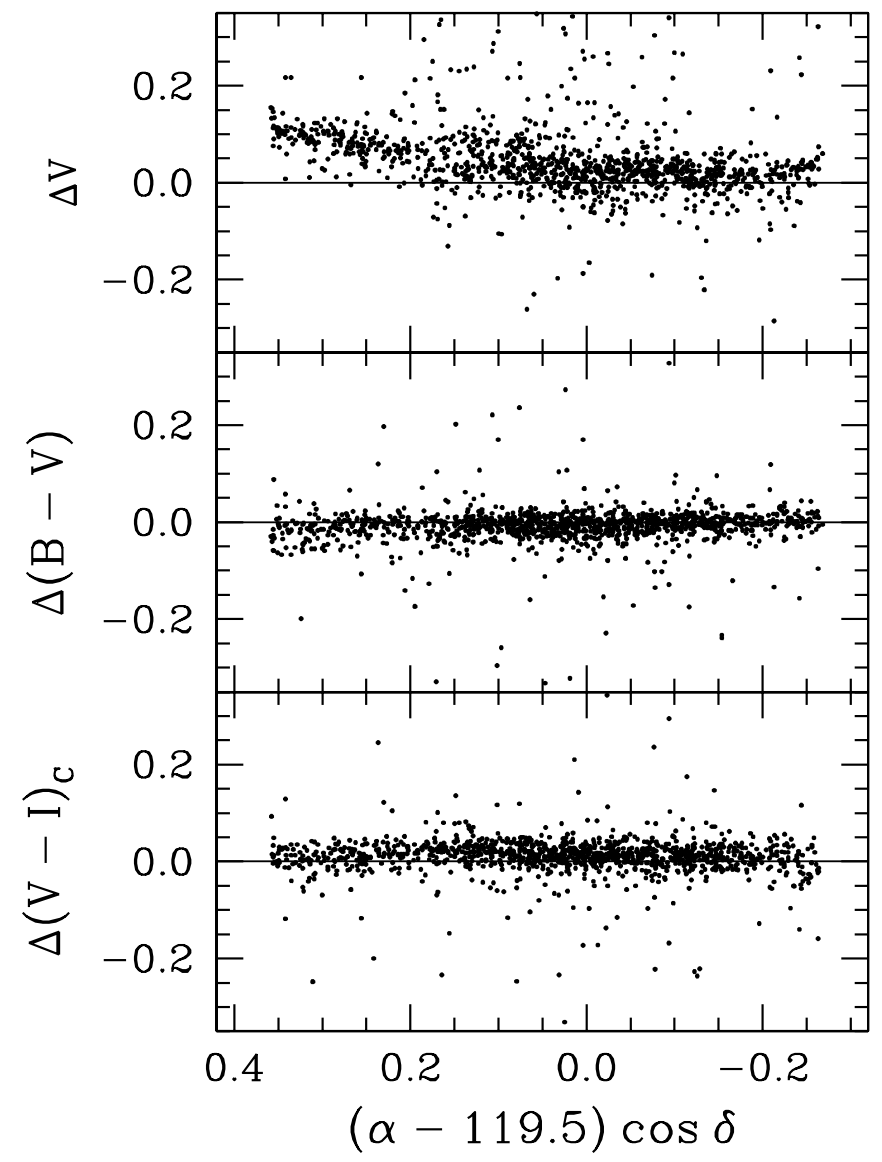

FIG. 1.-Comparison of NGC 2516 photometry as a function of right ascension (in degrees). The differences are in the sense of the S02 minus the JTH01 photometry.

error in $V, B-V$, and $V-I_{\mathrm{C}}$, respectively. In comparison to $\mathrm{S} 04$, the MMJ93 data are fainter in $V$ and are redder in $V-I_{\mathrm{C}}$. As shown in Figure 5 of S04, the differences are largest for those stars with $V-I_{\mathrm{C}} \lesssim 1.0$.

For NGC 2516, we have independent photometry in $B V I_{\mathrm{C}}$ from Jeffries et al. (2001, hereafter JTH01) and from Sung et al. (2002, hereafter S02). Neither study compares their photometry with the other. In Figure 1 we plot the differences in the photometry from the two studies against right ascension, which reveals a position-dependent difference in $V$ (but not in the colors). The sense of the difference is that the photometry toward the east is fainter in the S02 study. According to S02, photometry in this portion of the sky was obtained on a nonphotometric night, which was then adjusted to match the data in the rest of their survey using stars also observed under good conditions. As their paper lists only the average values, we chose to use the photometry only from regions obtained entirely under photometric conditions. However, there still remain significant differences, as shown in Figure 2, between the JTH01 and the S02 photometry even when the eastern data in the latter study are removed. Compared to the JTH01 values, the $\mathrm{S} 02$ data are brighter in $V$ and redder in $V-I_{\mathrm{C}}$ at the top of the MS but are fainter in $V$ and bluer in $B-V$ at the faint end. The mean differences are summarized in Table 2.

\subsubsection{Assignment of Random Errors}

Most of the collated photometry lacks errors for individual stars, and those errors reported by MMJ93, JTH01, and S02 are typically from a small number of repeat observations. Our MS fitting procedure $(\S 4.1)$ first removes stars that are statistically
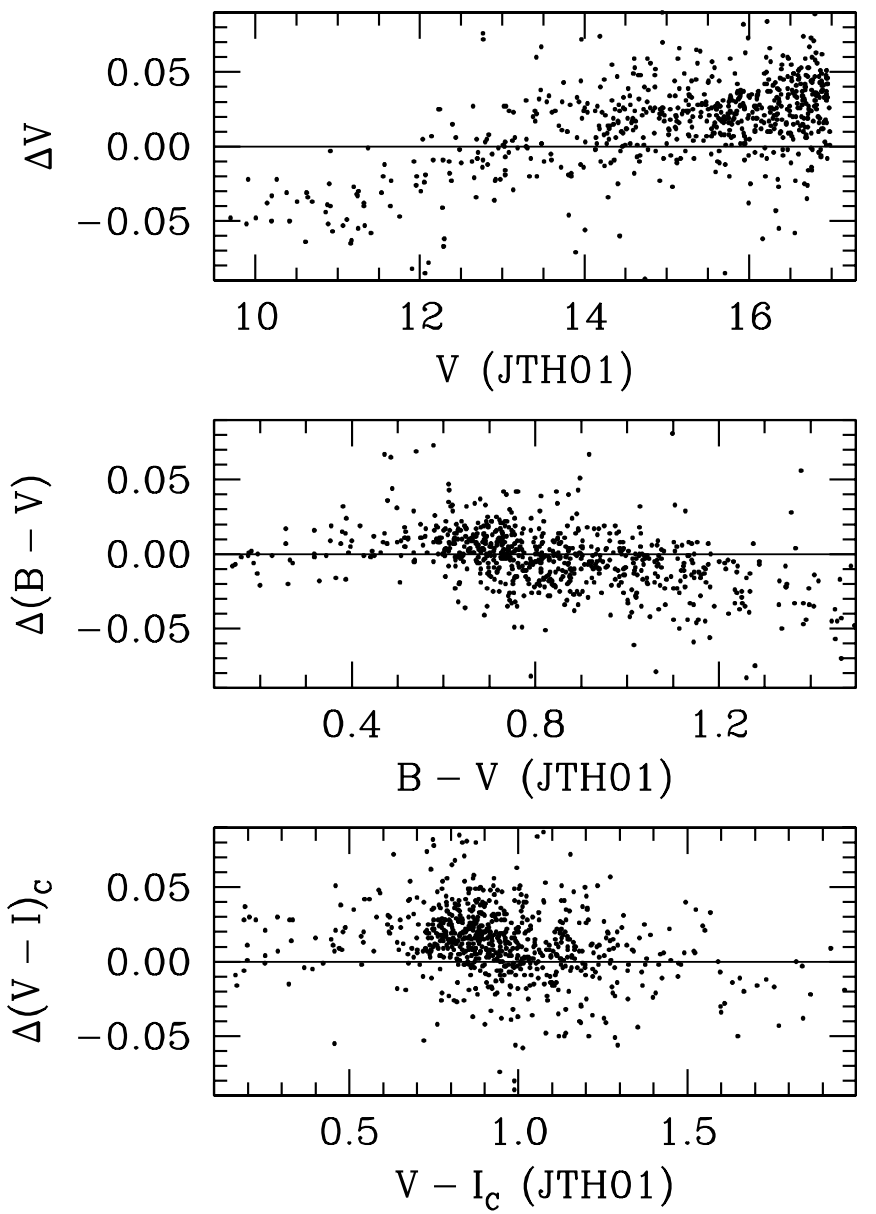

FIG. 2.-Comparison of NGC 2516 photometry, excluding stars observed under nonphotometric conditions by S02. The differences are in the sense of the S02 minus the JTH01 photometry.

separated from the MS, and this in turn requires a suitable error for each star.

In Praesepe and the Pleiades, we divided the data into bins in $V$, each 2 mag wide, and then computed the median of rms in magnitude and colors for the relatively few stars with multiple measurements. We assigned this median value to all data points in each bin as random photometric errors. For NGC 2516, we followed the same binning procedure, then removed a systematic trend in the differences between the JTH01 and S02 studies by subtracting a linear function in $V$. We then computed the rms of the differences in $V, B-V$, and $V-I_{\mathrm{C}}$ in each bin, divided these by $\sqrt{2}$, and assigned these errors to both data sets assuming that each study would have about the same errors. For M67, we followed the same procedure, first matching the stars by their coordinates, and computing the rms of the differences after removing a systematic trend in $V$. S04 reports the error for each star based on a large number of measurements. We used those errors directly in our analysis of the S04 data and assumed that the scatter between the two studies was dominated by measurement errors in MMJ93. We set the errors for the MMJ93 study from the rms of the differences with respect to the S04 data.

\subsection{4. $2 M A S S K_{S}$}

We calculated $V-K_{S}$ colors from the All Sky Data Release of the Two Micron All Sky Survey (2MASS) Point Source Catalog(PSC). ${ }^{7}$

\footnotetext{
${ }^{7}$ See http://www.ipac.caltech.edu/2mass/.
} 


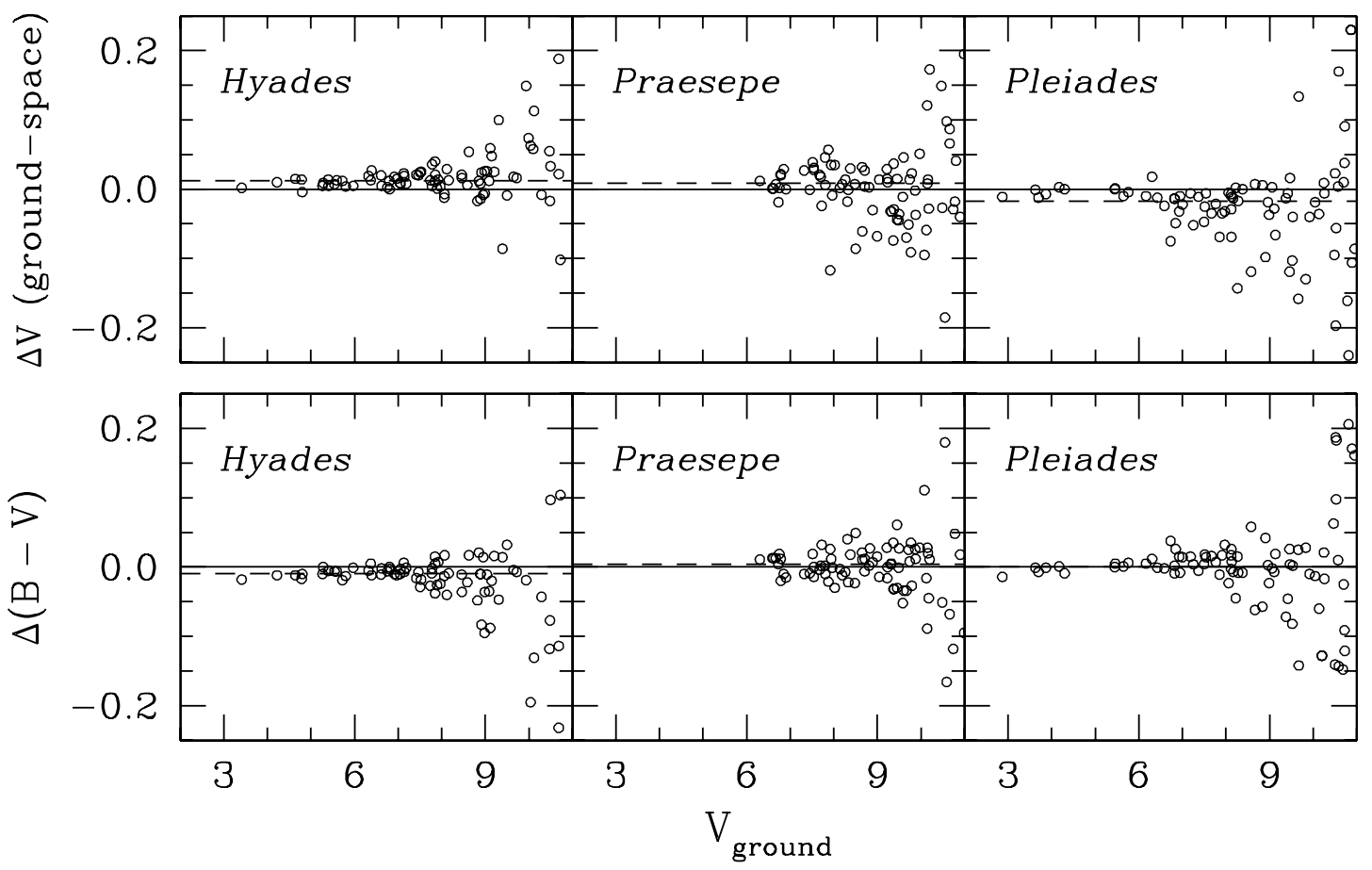

FIG. 3.-Differences in $V(t o p)$ and $B-V$ (bottom) between the ground-based and the Tycho-1 photometry, after transforming the latter into the Johnson system (see text). The differences are in the sense of the ground minus the Tycho-1 values. The solid line indicates equality, while the dashed line is plotted at the weighted mean difference for stars with $V_{T} \leq 9$ (Table 2). The scatter for $V>8$ mainly reflects errors in the Tycho photometry.

Here $K_{s}$ designates the "short"- $K$ filter in 2MASS (Carpenter 2001). Based on PSC flag parameters, we excluded stars that were saturated or undetected. We also ignored blended or contaminated sources. The $V-K_{S}$ errors were taken as the quadrature sum of $V$ errors and the catalog's "total" photometric uncertainties in $K_{s}$.

\subsection{Systematic Errors in the Photometry}

Our analysis of systematic errors in the MS fitting method ( $\S 6.1$ ) shows that calibration errors in the photometry contribute significantly to the overall error budget. In addition to the direct comparisons between the studies of M67 and NGC 2516, we can use the Tycho-1 photometry (van Leeuwen et al. 1997) to check whether photometry of the Hyades, Praesepe, and the Pleiades is on a consistent system (the other clusters are too distant to have many stars in the Tycho catalog). Here we assume that the Tycho photometry is consistently on the same scale in all parts of the sky. In Table 2, we display the mean differences between the ground-based photometry for these clusters and the Tycho values. The latter were transformed to the Johnson system using the equations in Oja \& Evans (1998) for $V$ and in Mamajek et al. (2002) for $B-V$. The values in the table were computed for stars with $V_{T} \leq 9$.

The comparison between the Tycho-1 values and the groundbased data is shown in Figure 3. The scatter is usually dominated by errors in the ground-based photometry for $V \leq 8$ and by the

TABLE 3

Differences in the Tycho Рнотомetry

\begin{tabular}{cccc}
\hline \hline Cluster & Comparison & $\left\langle\Delta B_{T}\right\rangle$ & $\left\langle\Delta V_{T}\right\rangle$ \\
\hline Hyades.......... & Tycho-2 - Tycho-1 & $-0.009 \pm 0.002$ & $-0.006 \pm 0.002$ \\
Praesepe ....... & Tycho-2 - Tycho-1 & $-0.018 \pm 0.004$ & $-0.025 \pm 0.004$ \\
Pleiades ........ & Tycho-2 - Tycho-1 & $-0.014 \pm 0.003$ & $-0.016 \pm 0.003$
\end{tabular}

Note.-Computed for stars with $V_{T} \leq 9$.
Tycho photometry for fainter stars. The solid line in each panel denotes equality, while the dashed line shows the mean difference from Table 2.

In Table 3 we list the differences between the Tycho- 1 and Tycho-2 data (Høg et al. 2000) for bright stars in the Hyades, Praesepe, and the Pleiades. The differences were computed directly with the Tycho values (again for $V_{T} \leq 9$ ), without transformations to the Johnson system. Taken together, the data in Tables 2 and 3 show that the various studies probably could have calibration errors on the order of 0.01-0.02 mag in $V$, but smaller in $B-V$. The situation in $V-I_{\mathrm{C}}$ is less well determined, mainly because we have fewer studies to compare.

In Paper II we also noted that the Tycho- 1 values for $V$ were brighter than the ground-based data by +0.012 ; we opted to add this constant to the Tycho data before averaging with the groundbased photometry. Had we chosen to adopt the space-based scale instead, our calibrated isochrones would be brighter in $V$ and also bluer in $V-K_{S}$ since the $V$ and $K_{S}$ data were obtained independently. This correction was not applied to the isochrones used in this paper. In the Appendix we discuss other issues involved in the isochrone calibration.

\subsection{Membership and Binarity}

We also collated information on binarity and membership in Praesepe and the Pleiades from WEBDA and the Open Cluster Database. Any star that was listed as a nonmember for any reason (e.g., from photometry or radial velocities) was rejected. In Praesepe, there are extensive data on binarity (e.g., Mermilliod \& Mayor 1999; Bouvier et al. 2001). Stars that were designated as likely or probable binary stars were flagged. In the Pleiades, a considerable number of stars have binarity and membership information (Jones 1981; Stauffer et al. 1991; Mermilliod et al. 1992; Schilbach et al. 1995; Bouvier et al. 1997; Belikov et al. 1998; Moraux et al. 2001). We rejected any star with a membership probability less than $50 \%$ but kept any star that did not 
TABLE 4

Spectroscopy in Praesepe

\begin{tabular}{|c|c|c|c|c|c|c|}
\hline $\mathrm{ID}^{\mathrm{a}}$ & $\begin{array}{l}T_{\text {eff }} \\
(\mathrm{K})\end{array}$ & $\begin{array}{c}\log g \\
\left(\mathrm{~cm} \mathrm{~s}^{-2}\right)\end{array}$ & $\begin{array}{c}\xi \\
\left(\mathrm{km} \mathrm{s}^{-1}\right)\end{array}$ & $\log \epsilon$ & $\sigma_{\log \epsilon}$ & {$[\mathrm{Fe} / \mathrm{H}]$} \\
\hline KW $23 \ldots \ldots \ldots \ldots$ & 5700 & 4.5 & 0.5 & 7.78 & 0.05 & 0.10 \\
\hline KW $58 \ldots \ldots \ldots . . . .$. & 5850 & 4.5 & 0.5 & 7.80 & 0.06 & 0.12 \\
\hline KW $304 \ldots \ldots \ldots . . .$. & 5625 & 4.4 & 0.4 & 7.80 & 0.06 & 0.12 \\
\hline KW $336 \ldots \ldots \ldots . . .$. & 5600 & 4.5 & 0.4 & 7.79 & 0.06 & 0.11 \\
\hline
\end{tabular}

${ }^{\mathrm{a}} \mathrm{KW}=$ Klein Wassink (1927).

have a membership probability. S04 provides a list of single cluster members based on proper-motion surveys and known binary systems in the literature and further elimination of unrecognized binaries in CMDs from his high-precision photometry. We used this information for both MMJ93 and S04 photometry in this paper, unless otherwise specified.

\subsection{Metallicity and Reddening}

\subsubsection{New Spectroscopy in Praesepe}

Obtaining accurate distances relative to our calibrating cluster, the Hyades, requires accurate relative metallicities because the luminosity of the MS is sensitive to the metal abundance. As part of this study, we determined a new metallicity for Praesepe using the same method used to find the Hyades abundance (Paulson et al. 2003). The relative abundance of Praesepe with respect to the Hyades should therefore be accurate since systematic errors arising from the solar abundances, employed model atmospheres, oscillator strengths, or effective temperature scales would be minimized.

We obtained spectra of four Praesepe stars with the Magellan Inamori Kyocera Echelle (MIKE) spectrograph (Bernstein et al. 2003) on the Magellan $6.5 \mathrm{~m}$ Clay telescope at Las Campanas Observatory. A $0.35^{\prime \prime}$ wide slit gave a resolving power of $\sim 55,000$ per resolution element (4 pixels) and wavelength coverage from 4500 to $9200 \AA$. The spectra are of high quality, with S/N > 100 .

The spectra were reduced using standard $\mathrm{IRAF}^{8}$ packages. The stellar parameters $-T_{\text {eff }}$, surface gravity $(\log g)$, microturbulence $(\xi)$, and $[\mathrm{Fe} / \mathrm{H}]$ - were derived following the procedure in Paulson et al. (2003) and are listed in Table 4. We employed the spectral synthesis code MOOG (Sneden 1973) and used stellar model atmospheres based on the 1995 version of the ATLAS9 code (Castelli et al. 1997). Within IRAF, we used Gaussian fits to $15 \mathrm{Fe}$ I lines and nine $\mathrm{Fe}$ II lines, a subsample of those listed in Table 1 of Paulson et al. (2003). The $T_{\text {eff }}$ was derived by requiring that individual line abundances be independent of excitation potential and that microturbulence $(\xi)$ be independent of line strength. Insisting on ionization equilibrium between $\mathrm{Fe}$ I and $\mathrm{Fe}$ II allowed for the simultaneous determination of $\log g$ with $T_{\text {eff }}$ and microturbulence $(\xi)$. Errors in $T_{\text {eff }}$ are $\sim 50 \mathrm{~K}$. We analyzed a reflected light spectrum of the asteroid Iris in order to obtain an instrumental correction to the solar $\log \epsilon(\mathrm{Fe})$. Using this correction, we obtained $[\mathrm{Fe} / \mathrm{H}]=0.11 \pm 0.03$ (s.e.m. ${ }^{9}$ ) for the Praesepe cluster.

\subsubsection{Metallicity and Reddening Estimates in the Literature}

For other clusters in this study, we adopted or calculated average metallicities from high-resolution spectroscopy as sum-

\footnotetext{
${ }^{8}$ IRAF is distributed by the National Optical Astronomy Observatory, which is operated by the Association of Universities for Research in Astronomy, Inc., under cooperative agreement with the National Science Foundation.

9 Standard error of the mean.
}

TABLE 5

Cluster Metallicity from High-Resolution Spectroscopy

\begin{tabular}{|c|c|c|}
\hline Reference & {$[\mathrm{Fe} / \mathrm{H}]$} & s.e.m \\
\hline \multicolumn{3}{|l|}{ Praesepe } \\
\hline Boesgaard \& Friel $^{\mathrm{a}}$ & +0.09 & 0.03 \\
\hline This paper (adopted value) .................. & +0.11 & 0.03 \\
\hline
\end{tabular}

\begin{tabular}{|c|c|c|}
\hline Cayrel et al. (1988) & +0.13 & 0.07 \\
\hline 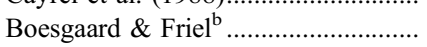 & +0.03 & 0.02 \\
\hline King et al. (2000) & +0.06 & 0.05 \\
\hline 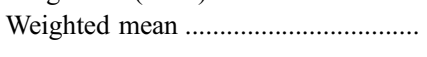 & +0.04 & 0.02 \\
\hline
\end{tabular}

\begin{tabular}{|c|c|c|}
\hline \multicolumn{3}{|l|}{ M67 } \\
\hline Garcia Lopez et al. (1988) .................. & +0.04 & 0.04 \\
\hline Hobbs \& Thorburn (1991) .................. & -0.06 & 0.03 \\
\hline Friel \& Boesgaard (1992).................... & +0.02 & 0.12 \\
\hline Tautvaišienè et al. (2000) ................... & -0.03 & 0.01 \\
\hline Yong et al. (2005) & +0.02 & 0.02 \\
\hline Randich et al. (2006) .............................. & +0.03 & 0.01 \\
\hline Weighted mean & $\begin{array}{c}+0.00 \\
\ldots\end{array}$ & $\begin{array}{c}0.01 \\
(0.03)^{c}\end{array}$ \\
\hline \multicolumn{3}{|l|}{ NGC 2516} \\
\hline Terndrup et al. (2002)........................... & +0.01 & 0.07 \\
\hline $\begin{array}{l}\text { a From Boesgaard (1989) and Frie } \\
\text { excluding known nonmembers. } \\
\text { b From Boesgaard (1989) and Boc } \\
\text { excluding known nonmembers. }\end{array}$ & \& Fri & $\begin{array}{l}\text { 2) after } \\
\text { 0) after }\end{array}$ \\
\hline
\end{tabular}

marized in Table 5. For the studies by Boesgaard \& Friel in Praesepe and the Pleiades, we have recomputed the cluster averages from individual $[\mathrm{Fe} / \mathrm{H}]$ estimates after excluding cluster nonmembers. The only existing measurement of the abundance of NGC 2516 using high-resolution spectroscopy is from Terndrup et al. (2002), which was derived from only two stars.

Table 6 lists reddening estimates from a variety of sources. For the Pleiades, we adopted a uniform reddening inferred from the $\mathrm{H}$ I hole measurement by Breger (1986). Although small parts of the cluster show higher reddening (e.g., Eggen 1950; Mitchell \& Johnson 1957), the stars in these regions constitute only a small fraction of our Pleiades sample, and most of these were tagged as outliers from our filtering algorithm ( $\$ 4.1)$. We compared magnitudes and colors derived from individual $E(B-V)$ corrections for 157 stars (Breger 1986; Stauffer \& Hartmann 1987; Soderblom et al. 1993) with those from the uniform reddening, but the differences were negligible in our MS fitting range ( $§ 4.2)$. The reddening measurements for the Pleiades from other studies in Table 6 likely represent a cluster average including the $\mathrm{CO}$ cloud region.

\section{CALIBRATED ISOCHRONES}

We used the YREC to construct stellar evolutionary tracks at $-0.3 \leq[\mathrm{Fe} / \mathrm{H}] \leq+0.2$ in 0.1 dex increments. At each metal abundance, we ran a grid of masses from 0.2 to $8 M_{\odot}$ in $0.05-$ $1 M_{\odot}$ increments depending on the stellar mass. The scaled solar abundance mix from Grevesse \& Sauval (1998) and the helium enrichment parameter $\Delta Y / \Delta Z=1.2$ from nondiffusion models were used; see Sills et al. (2000) and Paper I for detailed information. The tracks were interpolated to generate theoretical isochrones at stellar ages from $20 \mathrm{Myr}$ to $4 \mathrm{Gyr}$. 
TABLE 6

Cluster Reddening in the Literature

\begin{tabular}{|c|c|c|c|}
\hline Reference & Method & $E(B-V)$ & s.e.m. \\
\hline \multicolumn{4}{|c|}{ Praesepe } \\
\hline Mermilliod (1981b) & $U B V$ & 0.00 & $0.01^{\mathrm{a}}$ \\
\hline Nicolet (1981) & Geneva & 0.011 & 0.005 \\
\hline 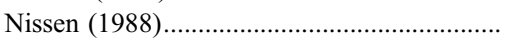 & $u v b y-\beta^{\mathrm{b}}$ & 0.007 & 0.002 \\
\hline \multirow[t]{2}{*}{ Weighted mean } & $\ldots$ & 0.007 & 0.002 \\
\hline & $\ldots$ & $\ldots$ & $(0.003)^{\mathrm{c}}$ \\
\hline \multicolumn{4}{|c|}{ Pleiades } \\
\hline Mermilliod (1981b) & $U B V$ & 0.04 & $0.01^{\mathrm{a}}$ \\
\hline Nicolet (1981) & Geneva & 0.062 & 0.005 \\
\hline Breger $(1986)^{d} \ldots \ldots \ldots \ldots$ & Sp-type, $u v b y-\beta^{\mathrm{b}}$ & 0.044 & 0.003 \\
\hline 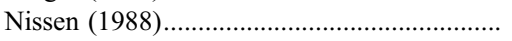 & $u v b y-\beta^{\mathrm{b}}$ & 0.039 & 0.005 \\
\hline \multirow[t]{2}{*}{ Weighted mean ..................................................... } & $\ldots$ & 0.046 & 0.005 \\
\hline & & & $(0.009)^{\mathrm{c}}$ \\
\hline Breger (1986) (adopted value) ${ }^{\mathrm{e}}$.......................... & Sp-type, $u v b y-\beta^{\mathrm{b}}$ & 0.032 & 0.003 \\
\hline \multicolumn{4}{|c|}{ M67 } \\
\hline Taylor (1980) & Multiple approaches & 0.046 & 0.006 \\
\hline 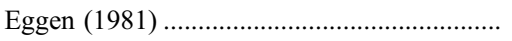 & $u v b y-\beta^{\mathrm{b}}$ & 0.050 & 0.013 \\
\hline 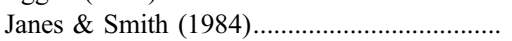 & DDO & 0.056 & 0.006 \\
\hline 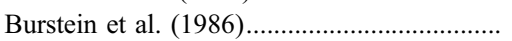 & BH map ${ }^{\mathrm{f}}$ & $0.035^{\mathrm{g}}$ & 0.005 \\
\hline Nissen et al. (1987) & $u v b y-\beta^{\mathrm{b}}$ & 0.032 & 0.006 \\
\hline 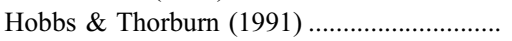 & $T_{\text {eff }}-(B-V)_{0}$ & 0.065 & 0.018 \\
\hline Montgomery et al. (1993) .................................. & $U B V$ & 0.05 & 0.01 \\
\hline 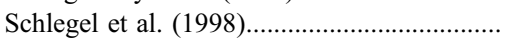 & Dust map & 0.032 & $0.005^{\mathrm{h}}$ \\
\hline \multirow[t]{2}{*}{ 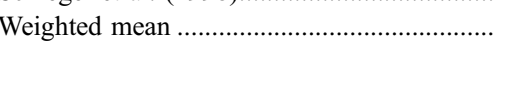 } & $\ldots$ & 0.041 & 0.004 \\
\hline & $\ldots$ & $\ldots$ & $(0.010)^{\mathrm{c}}$ \\
\hline \multicolumn{4}{|c|}{ NGC 2516} \\
\hline Eggen (1972) & $U B V$ & 0.125 & 0.025 \\
\hline Feinstein et al. (1973) & Sp-type, $U B V$ & 0.116 & 0.004 \\
\hline 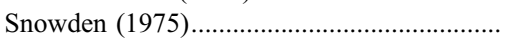 & $u v b y-\beta^{\mathrm{b}}$ & 0.120 & 0.014 \\
\hline 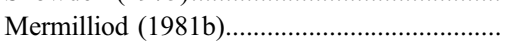 & $U B V$ & 0.11 & $0.01^{\mathrm{a}}$ \\
\hline 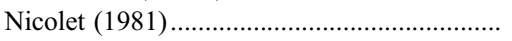 & Geneva & 0.114 & 0.012 \\
\hline 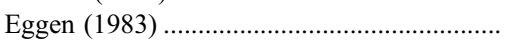 & $u v b y-\beta^{\mathrm{b}}$ & 0.118 & 0.004 \\
\hline Verschoor \& van Genderen (1983) ................. & $V B L U W$ & 0.127 & 0.005 \\
\hline 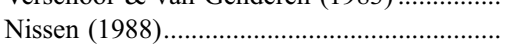 & $u v b y-\beta^{\mathrm{b}}$ & 0.109 & 0.007 \\
\hline 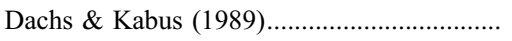 & Sp-type, $U B V$ & 0.12 & 0.004 \\
\hline Sung et al. (2002) & $U B V$ & 0.112 & 0.006 \\
\hline \multirow[t]{2}{*}{ 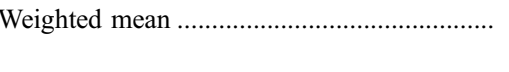 } & $\ldots$ & 0.117 & 0.002 \\
\hline & $\ldots$ & $\ldots$ & $(0.005)^{\mathrm{c}}$ \\
\hline
\end{tabular}

${ }^{\mathrm{a}}$ Assigned value in this paper.

${ }^{\mathrm{b}} E(B-V)=E(b-y) / 0.74$ (Cousins \& Caldwell 1985).

${ }^{c}$ Standard deviation of the measurements.

d Average over the cluster field.

e Average over the $\mathrm{H}$ i hole (see text).

${ }^{\mathrm{f}}$ Burstein \& Heiles (1982).

${ }^{g}$ Corrected for the systematic difference with Schlegel et al. (1998) of $0.02 \mathrm{mag}$.

${ }^{\mathrm{h}}$ Quoted precision (16\%).

Stellar luminosities and $T_{\text {eff }}$ were initially converted to $V$, $B-V, V-I_{\mathrm{C}}$, and $V-K_{s}$ from the relations by Lejeune et al. $(1997,1998)$. To obtain finer grids of the isochrones, we increased the $M_{V}$ spacing of the isochrone points using a cubic spline to match the $\Delta M_{V}=0.05$ spacing of our tabulated color- $T_{\text {eff }}$ corrections in Paper II. We then linearly interpolated the isochrones in both $[\mathrm{Fe} / \mathrm{H}]$ and age at a fixed $M_{V}$. We finally applied the empirical color corrections, as defined in Paper II and updated in the Appendix of this paper. We assumed that the correction table is valid over the entire model grid in $[\mathrm{Fe} / \mathrm{H}]$ and age.
The isochrones constructed in this way are available online. ${ }^{10}$

\section{DISTANCES AT FIXED REDDENING}

We now proceed to derive the cluster distances, adopting the reddening values from Table 6 . We do this in two ways, either holding the metallicity fixed at the spectroscopic values (Table 5) or determining a photometric metallicity that brings the distances

10 See http://www.astronomy.ohio-state.edu/iso/. 
from different CMDs into agreement. In either case, we show that the calibrated isochrones improve the internal precision of distance estimates from the three CMDs with $B-V, V-I_{\mathrm{C}}$, and $V-K_{s}$ as color indices and $V$ as a luminosity index, hereafter $(B-V, V),\left(V-I_{\mathrm{C}}, V\right)$, and $\left(V-K_{s}, V\right)$, respectively.

\subsection{Photometric Filtering}

Before fitting isochrones, we identified and removed stars that are far from the MS in comparison to the sizes of their photometric errors. Such stars could be either foreground/background objects or cluster binaries that stand off from the MS.

The filtering process iteratively identifies the MS as the locus of points of maximum density on the CMDs, independently of the isochrones. As an initial step, we identified the MS by hand and then removed stars more than 1 mag away in $V$. This was necessary in particular for the analysis in NGC 2516, which shows many faint background stars in its CMDs (see below) that would complicate finding the density maxima. The generous rejection criterion of 1 mag was chosen so that all cluster binaries would be preserved at this stage.

At each step of the iteration, data points on each CMD were sorted by $V$ magnitude into nonoverlapping groups: each group contained $\sqrt{N}$ points, where $N$ is the number of data points within a color range used in the filtering. The median colors in each group were computed, and then the resulting run of points ( $V$ vs. color) was made into a smooth curve by averaging each point with the linear interpolation of adjacent points.

With the above trial MS, we computed $\chi^{2}$ from each CMD as

$$
\chi^{2}=\sum_{i=1}^{N} \chi_{i}^{2}=\sum_{i=1}^{N} \frac{\left(\Delta V_{i}\right)^{2}}{\sigma_{V, i}^{2}+\left(\gamma_{i} \sigma_{c, i}\right)^{2}+\sigma_{0}^{2}},
$$

where $\Delta V_{i}$ is the $V$ difference between the $i$ th data point and MS at the color of the star and $\sigma_{V, i}$ and $\sigma_{c, i}$ are the photometric errors in $V$ and color, respectively. The error in the color contributes to the error in $\Delta V_{i}$ by the slope of the curve, $\gamma_{i}$, which was evaluated at the star's color. Because the above-constructed curve would not precisely trace the observed MS in the presence of outliers, we added $\sigma_{0}$ in quadrature to the propagated photometric errors in the denominator. We adjusted the value of $\sigma_{0}$ so that the total $\chi^{2}$ is equal to $N$. Initially, we rejected all data points as the MS outliers if the $\chi_{i}^{2}$ (the individual contribution to $\chi^{2}$ ) is greater than 9 (corresponding to a $3 \sigma$ outlier). We repeated adjusting $\sigma_{0}$ and rejecting outliers with the reduced set of data points until there remained no point with $\chi_{i}^{2}$ greater than the above threshold value. We combined the results from all three CMDs and rejected stars if they were tagged as an outlier from any one of the CMDs.

We imposed an additional condition for the convergence of the algorithm because cluster binaries and foreground/background stars are often substantial populations near the MS. We compared the rms of $\Delta V_{i}$ with its median of all absolute deviations (MAD). The MAD of a quantity $x_{i}$ is defined by

$$
\operatorname{MAD}=1.483 \operatorname{median}\left(\left|x_{i}-\operatorname{median}\left(x_{i}\right)\right|\right),
$$

where the correction factor 1.483 makes the estimator consistent with the standard deviation for a normal distribution (Rousseeuw 1990). The MAD is a more robust estimator of the dispersion than the standard deviation in the presence of outliers. Therefore, we reduced the size of the $\chi_{i}^{2}$ threshold value if the fractional difference between the rms and the MAD of $\Delta V_{i}$ is larger than $5 \%$, assuming median $\left(\Delta V_{i}\right)=0$. We repeated the above filtering

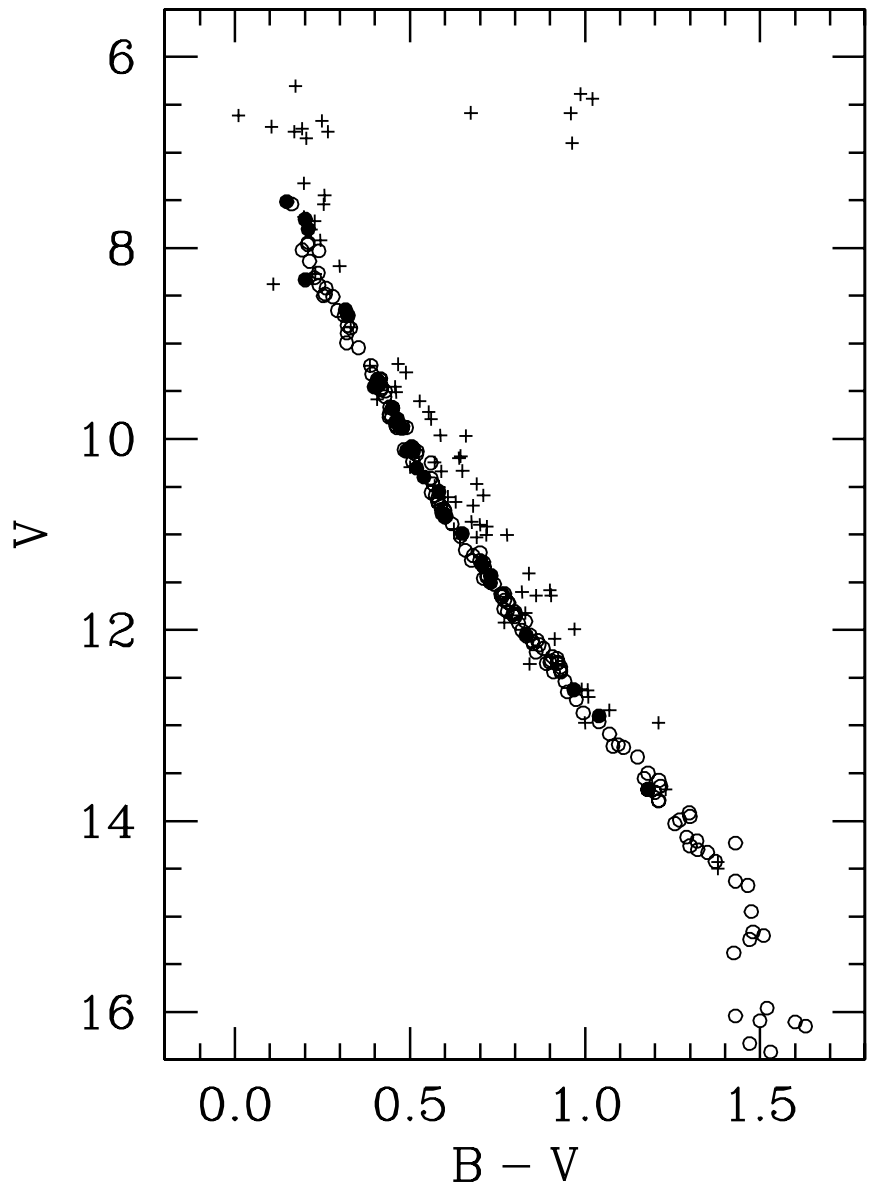

FIG. 4.- Result of the filtering process in Praesepe. All known cluster binaries were included to test the filtering algorithm. The plus signs are rejected points from the filtering, and open circles are those remaining. Filled circles are known binaries that remained after the filtering. Stars brighter than $V=7$ were excluded before the filtering algorithm was applied.

steps by constructing a new MS curve from the remaining data set.

Figure 4 illustrates the result of the filtering algorithm in Praesepe. Here we include all known cluster binaries but exclude stars with a low membership probability $(\S 2.4)$. We used all three CMDs in the filtering, but only the $(B-V, V)$ diagram is shown. The plus signs are rejected points from the filtering, and open circles are those remaining. Some of the stars on the MS were rejected because they were filtered in other CMDs. Although many binaries remain after the filtering (filled circles), their proximity to the MS would have a minor impact on the derived distance. The bias in the distance due to the remaining binaries is discussed in $\S 6.2$ from artificial cluster CMD tests.

\subsection{Isochrone Fitting and the Photometric Metallicity}

The cluster distances were found by fitting isochrones over a range of metallicity with the adopted age of each cluster $(\S 2.1)$. The isochrones were reddened to the $E(B-V)$ values in Table 6 . We adopted a reddening prescription for the broadband colors by Bessell et al. (1998), who have assumed the extinction law of Mathis (1990). Their reddening formulae were computed from ATLAS9 synthetic stellar photometry for a wide range of $T_{\text {eff }}$ and include color-dependent reddening resulting from shifts in the effective wavelengths of broadband filters. The Bessell et al. (1998) formulae give reddening and extinction values for $E(B-V)=$ 0.30 , so we linearly rescaled them according to the assumed 
cluster reddening. The color transformation by Carpenter $(2001)^{11}$ was used to compute the reddening in $V-K_{s}$. For zero-color stars, we found $R_{V} \equiv A_{V} / E(B-V)=3.26, R_{V I} \equiv E\left(V-I_{\mathrm{C}}\right) /$ $E(B-V)=1.32$, and $R_{V K} \equiv E\left(V-K_{S}\right) / E(B-V)=2.91$. For stars in the middle of our MS fitting range, $(B-V)_{0}=0.8$, we found $R_{V}=3.44, R_{V I}=1.37$, and $R_{V K}=3.04$.

For a given isochrone, we computed each individual star's distance modulus $\left(\mu_{i}\right)$ and defined the cluster distance modulus in each CMD as the "unweighted" median of $\mu_{i}$, i.e., $(m-M)_{0} \equiv$ median $\left(\mu_{i}\right)$. We computed the fitting error in the distance modulus on each CMD as

$$
\sigma_{(m-M)}=\max \left[\sigma_{\mathrm{phot}}, \frac{\operatorname{MAD}\left(\mu_{i}\right)}{\sqrt{N}}\right],
$$

where $N$ is the total number of data points used in the fit. Here

$$
\frac{1}{\sigma_{\text {phot }}^{2}}=\sum_{i=1}^{N} \frac{1}{\sigma_{V, i}^{2}+\left(\gamma_{i} \sigma_{c, i}\right)^{2}},
$$

where the same notation is used as in equation (1) except that $\gamma_{i}$ is the isochrone slope at the color of the star. We further computed a "weighted" mean and a "weighted" median of the distance modulus and discuss the difference between the three distance estimates along with other systematic errors in $\S 6.1$.

For a given set of $[\mathrm{M} / \mathrm{H}]$, age, and $E(B-V)$, we fit isochrones in $(B-V, V),\left(V-I_{\mathrm{C}}, V\right)$, and $\left(V-K_{s}, V\right)$ over $0.4 \leq(B-$ $V)_{0} \leq 1.3$. This color range is where the Hyades calibration is most reliable (Paper II). There were only a few Hyades members blueward of the range, and the cool end was set by the magnitude limit of the Hipparcos mission. The range corresponds to $0.48 \leq$ $\left(V-I_{\mathrm{C}}\right)_{0} \leq 1.48$ and $0.98 \leq\left(V-K_{S}\right)_{0} \leq 3.16$. For nonzero reddening, the fitting ranges were made correspondingly redder.

We define the photometric metallicity $[\mathrm{M} / \mathrm{H}]_{E}$ as the one that brings distances from three CMDs into statistical agreement (e.g., Pinsonneault et al. 1998; Stello \& Nissen 2001; Terndrup et al. 2002). Figure 5 shows how this process works for the Hyades, our calibrating cluster. The open circles display the derived distances as a function of isochrone metallicity in each of the three CMDs with $E(B-V)=0.000$ at an age of 550 Myr. The lines connecting these points are least-squares fits, and the labels to the left of the lines indicate the corresponding color index. The slope in the $(B-V, V)$ is larger than in the other two CMDs, indicating a greater sensitivity of the isochrone luminosity to the metal abundance. We define $[\mathrm{M} / \mathrm{H}]_{E}$ as the weighted average of the two metallicities at which the $(B-V, V)$ distance agrees with that from $\left(V-I_{\mathrm{C}}, V\right)$ and from $\left(V-K_{s}, V\right)$, respectively. For the Hyades in Figure 5, we derive the photometric metallicity of $[\mathrm{M} / \mathrm{H}]_{E}=+0.13 \pm 0.02$, which is naturally the same as the originally assumed value in the isochrone calibration.

With this definition of the photometric metallicity, we can derive two distances at the adopted reddening. The first of these, designated as $(m-M)_{0, S}$, is the weighted average distance modulus from the three CMDs at the spectroscopic $[\mathrm{Fe} / \mathrm{H}]$. The second distance, $(m-M)_{0, E}$, is the value determined at the photometric metallicity, $[\mathrm{M} / \mathrm{H}]_{E}$.

\subsection{Results}

Figures 6-9 display the CMDs for each cluster. For Praesepe and the Pleiades, known binaries and nonmembers were ex-

\footnotetext{
11 Updated color transformations for 2MASS All-Sky Data Release can be found at http://www.ipac.caltech.edu/2mass/releases/allsky/doc/sec6_4b.html.
}

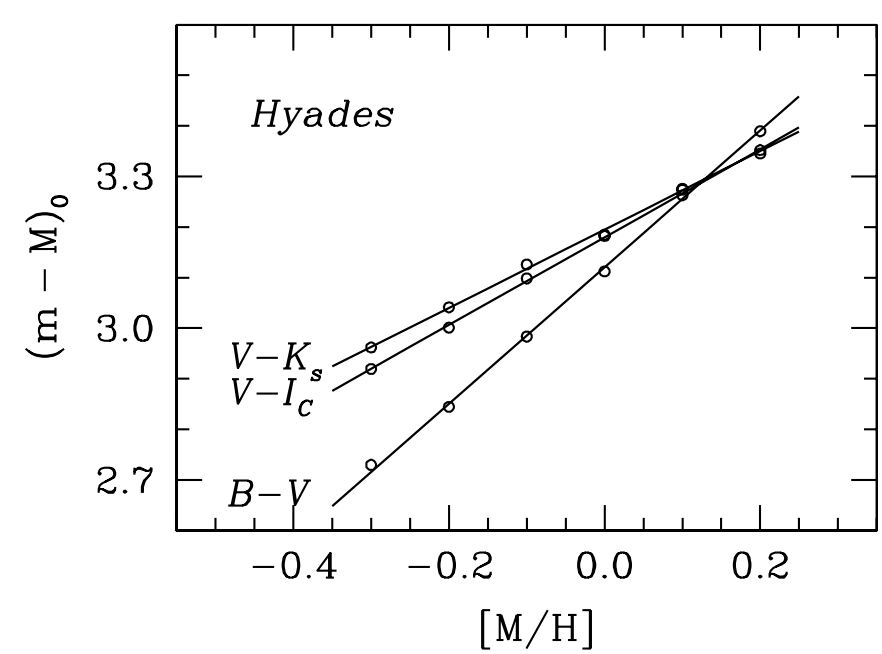

FIG. 5.-Example of determining a photometric metallicity in the Hyades. Open circles are the distances derived from CMDs with $B-V, V-I_{\mathrm{C}}$, and $V-K_{s}$ as a function of isochrone metallicity. The solid lines connecting these points are least-squares fits, and the labels to the left of the lines indicate the corresponding color index. An age of $550 \mathrm{Myr}$ and $E(B-V)=0.000$ were assumed. The photometric metallicity is defined as the average $[\mathrm{M} / \mathrm{H}]$ where the $B-V$ line crosses the $V-I_{\mathrm{C}}$ and $V-K_{s}$ lines.

cluded before we applied the photometric filtering. Stars that remained after the filtering are shown as open circles, and the rejected stars are shown as plus signs. With the extensive binary and membership information, the CMDs of Praesepe (Fig. 6) are dominated by single cluster members, and there are few stars rejected by the photometric filtering. The CMDs of the Pleiades (Fig. 7) show that the filtering routine identified likely cluster binaries effectively. The single cluster members from S04 are shown in the CMDs of M67 (Fig. 8), none of which were rejected by the filtering algorithm. The CMDs of NGC 2516 (Fig. 9) are from JTH01 and have a large number of foreground/background stars as expected in an area survey (for clarity, the point size for the rejected stars was reduced on the CMDs as compared to the other plots).

Isochrones at the spectroscopic metallicities are overlaid as solid lines in each figure, and in most cases they are in excellent agreement with the observed shapes of the MS. In particular, the match to the Praesepe MS (Fig. 6), which has an age and metallicity nearly identical to the Hyades, is good over almost 7 mag in $V$. We interpret this agreement as indicating that the Hyadesbased corrections to the color- $T_{\text {eff }}$ relations defined in Paper II apply to all clusters, or at least to those with metallicities not too different from that of the Hyades.

There are a few cases in which the match to the MS shape is not as good as it is in Praesepe. In the Pleiades (Fig. 7), stars with $B-V \gtrsim 0.9$ are considerably bluer than the isochrone in $(B-$ $V, V)$, although not in the other two CMDs. This "blue K dwarf" phenomenon was discussed by Jones (1972), Landolt (1979), and van Leeuwen et al. (1987) and at greater length by Stauffer et al. (2003), who attributed it to stellar temperature inhomogeneities caused by cool spots and plage areas in rapidly rotating young stars. From the JTH01 photometry, it would appear that NGC 2516 , which is about $40 \%$ older than the Pleiades, does not share this phenomenon with the Pleiades (see Fig. 9). However, we show in $\S 7.1$ that the usage of the S02 data comes to a different conclusion with supporting evidence from stellar rotational velocities. The data in $\left(V-I_{\mathrm{C}}, V\right)$ for all clusters are somewhat bluer than the isochrone redward of $V-I_{\mathrm{C}} \approx 1.6$; this indicates a possible systematic error in the Hyades calibration for these red 


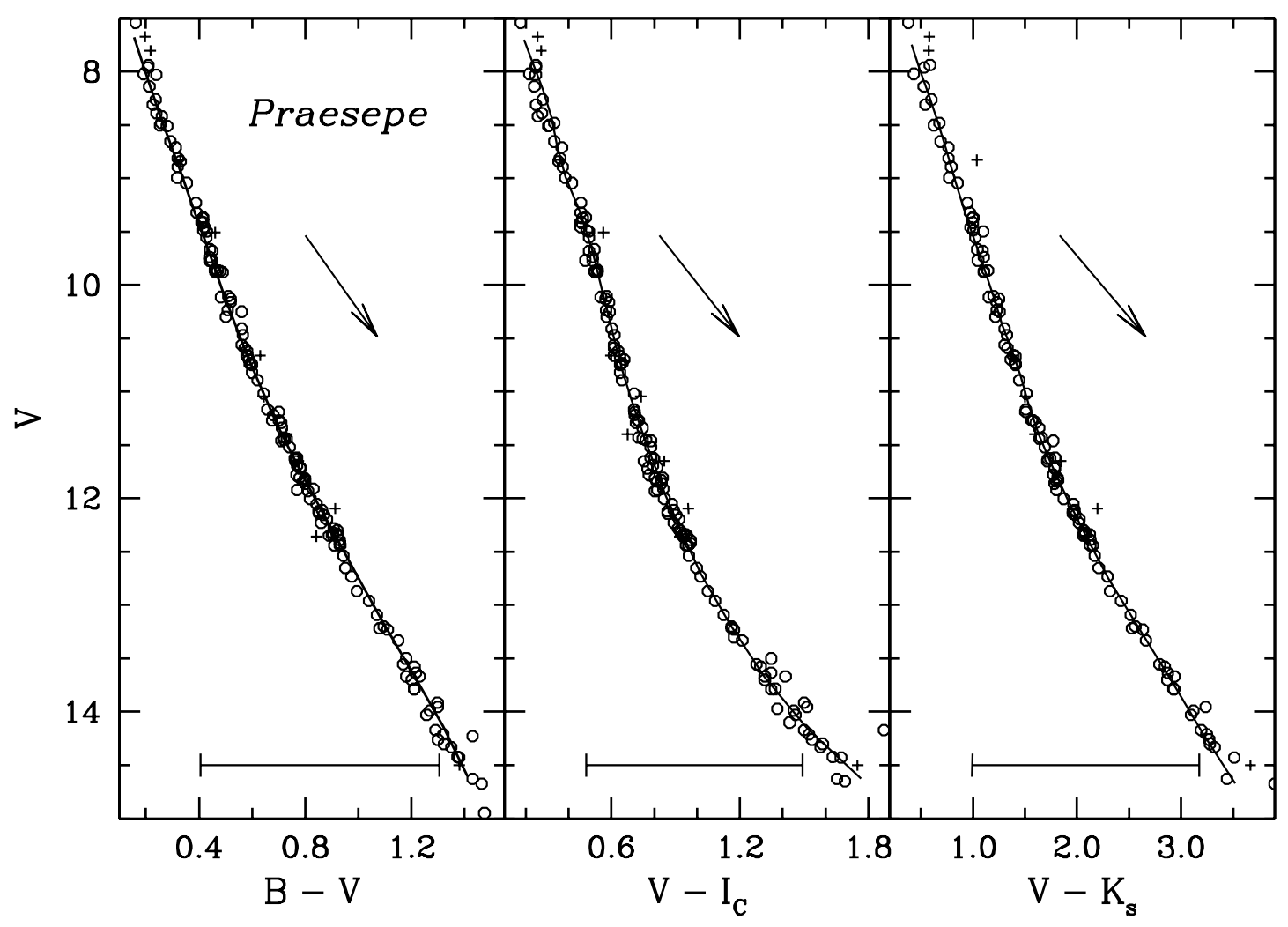

FIG. 6.-CMDs of Praesepe, after excluding known binaries and stars with low membership probability. Plus signs are photometrically rejected data points from the filtering algorithm, and open circles are those remaining. The solid lines are empirically calibrated isochrones with spectroscopic metallicity ( Table 5), which were adjusted for the reddening with the literature value (Table 6). Fitting ranges are shown as horizontal bars. The arrow denotes the direction of reddening vectors.

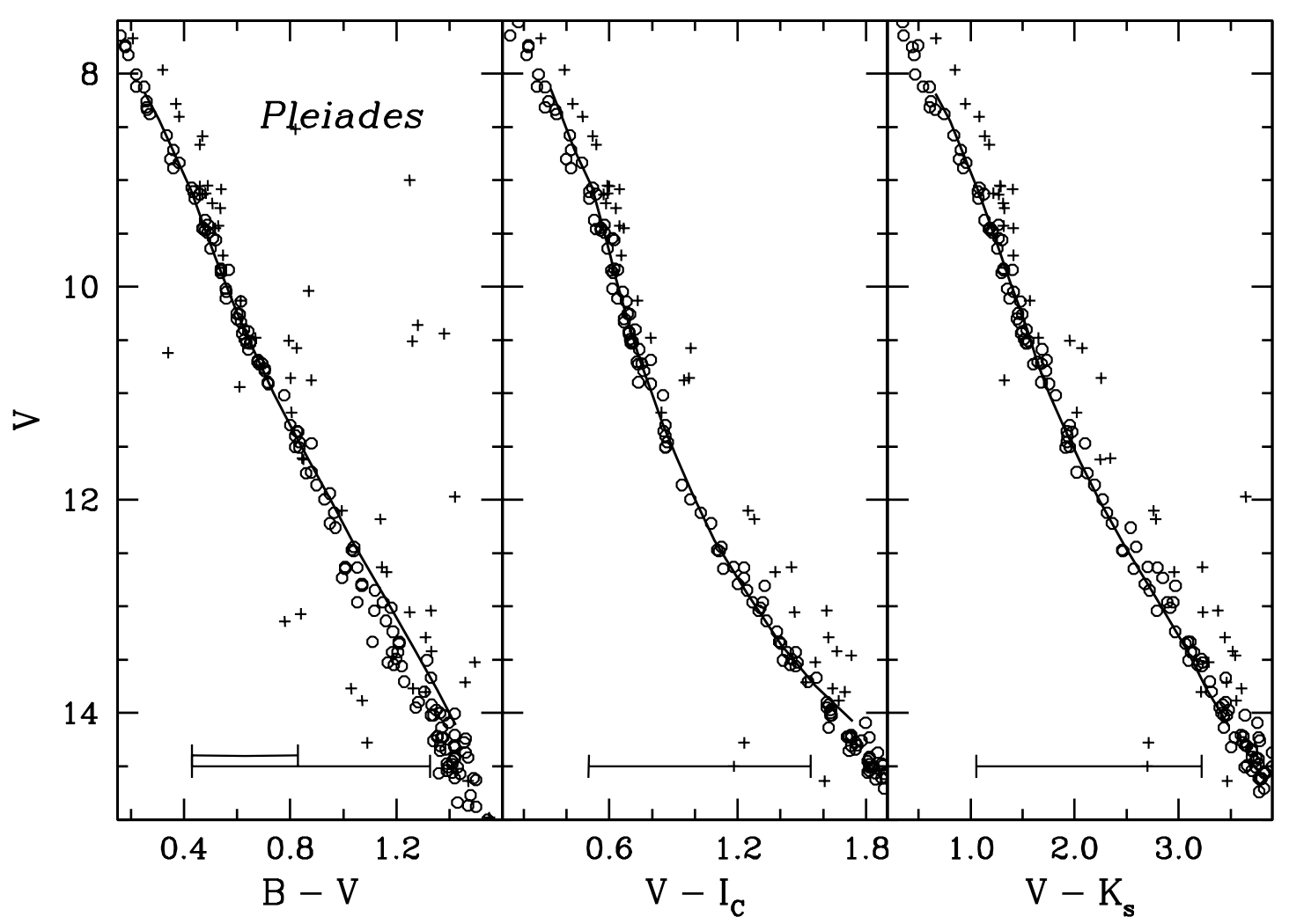

Fig. 7. - Same as Fig. 6, but for the Pleiades. As discussed by Stauffer et al. (2003), the Pleiades K dwarfs are bluer than the given isochrone in $(B-V, V)$, but not in the other two CMDs (see text). The distance modulus in $(B-V, V)$ was derived at $0.4 \leq(B-V)_{0} \leq 0.8$ as shown by the shorter horizontal bar. 


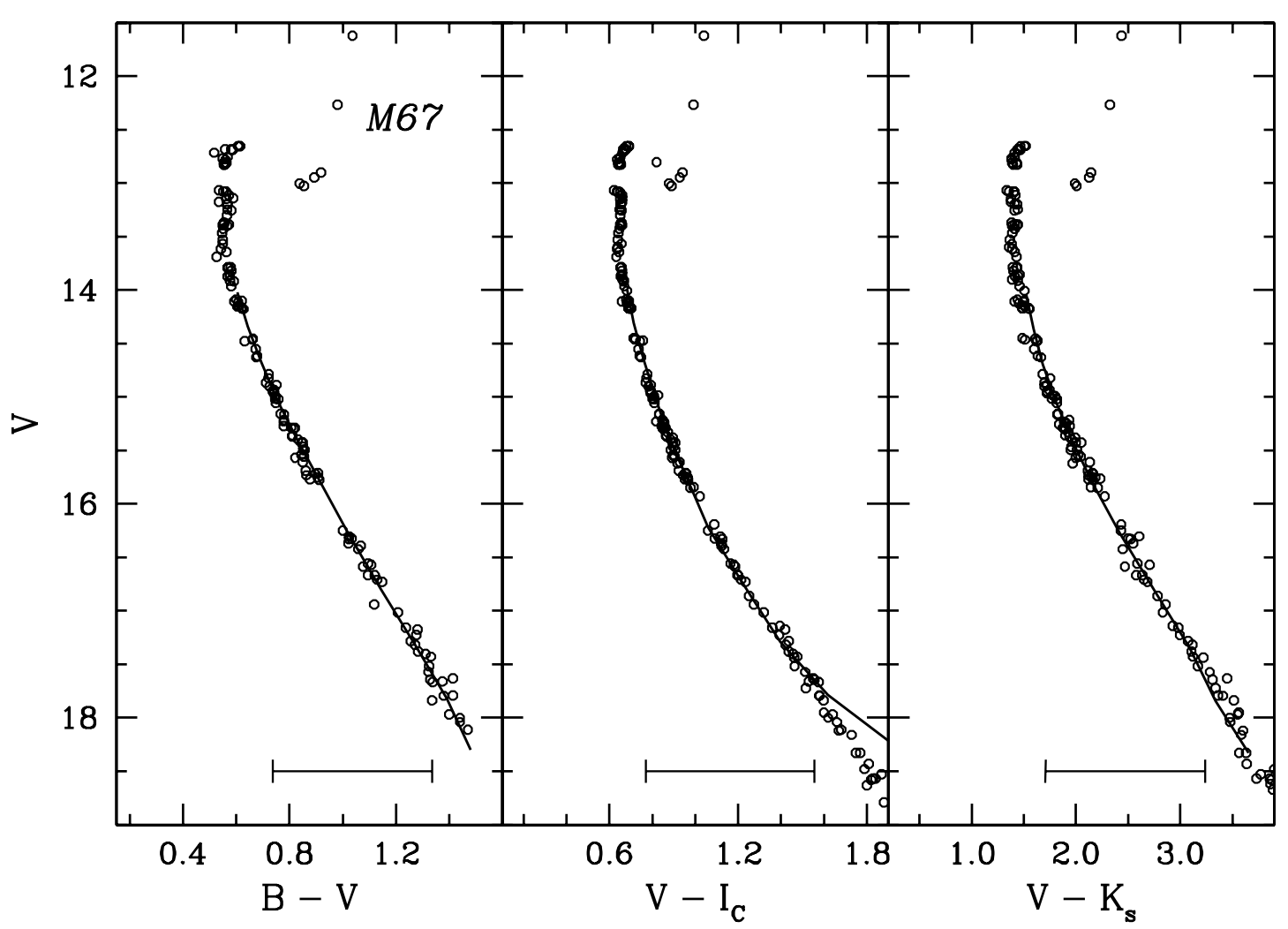

FIG. 8.- Same as Fig. 6, but for M67 with photometry from S04. Shown are those stars designated as single cluster members by S04. The fit was performed over $0.7 \leq(B-V)_{0} \leq 1.3$ and corresponding color ranges in the other two CMDs to avoid the steeply rising part of the upper MS.

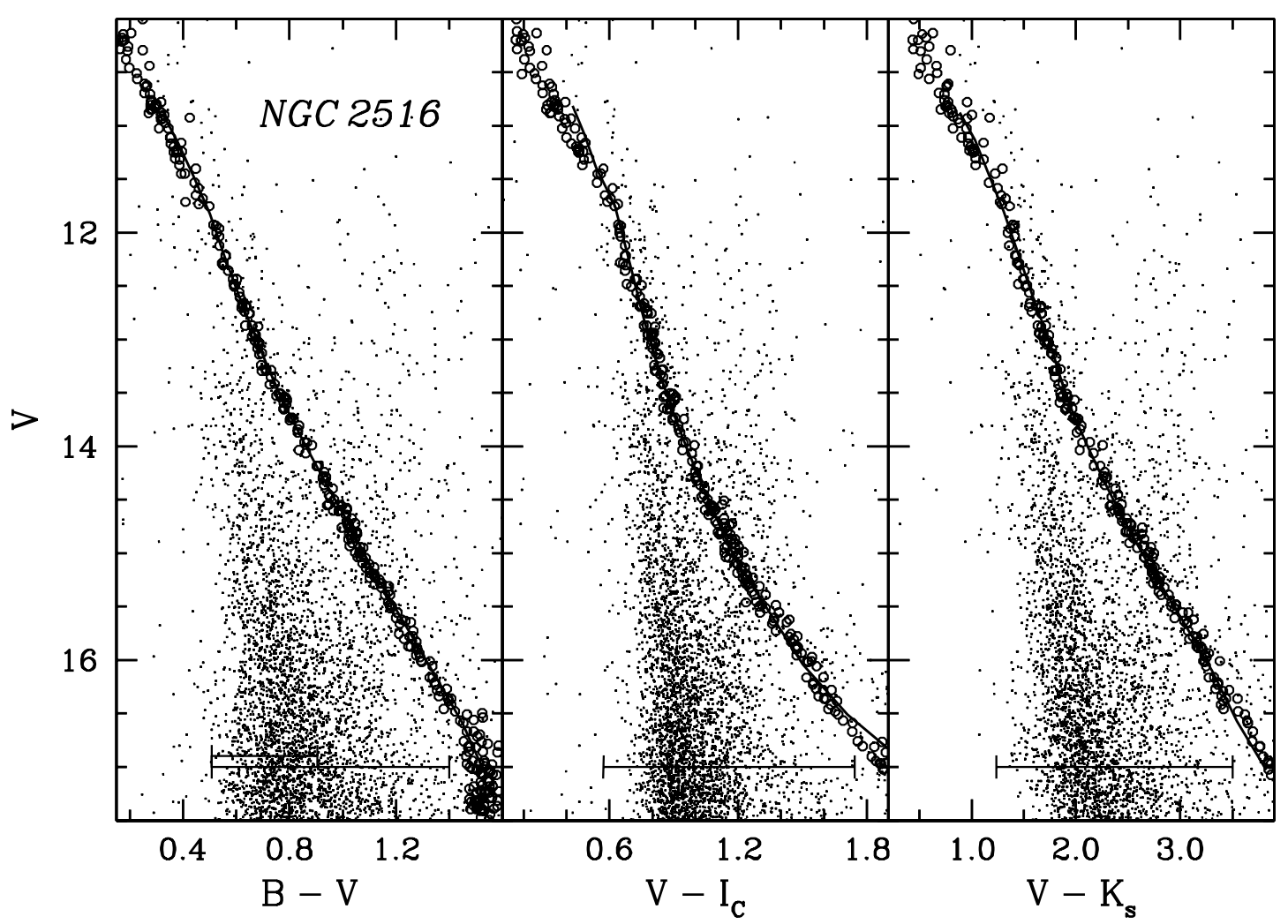

FIG. 9.- Same as Fig. 6, but for NGC 2516 with photometry from JTH01. The fitting range in $(B-V, V)$ was truncated in the red part as in the Pleiades CMD (see text). 
TABLE 7

MS Fitting Distance and Photometric Metallicity at Literature $E(B-V)$

\begin{tabular}{|c|c|c|c|c|c|c|}
\hline Data/Constraint & \multicolumn{3}{|c|}{$(m-M)_{0}$} & $(m-M)_{0, S}$ & {$[\mathrm{M} / \mathrm{H}]_{E}$} & $(m-M)_{0, E}$ \\
\hline 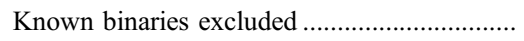 & $6.267 \pm 0.008$ & $6.291 \pm 0.008$ & $6.336 \pm 0.007$ & $6.303 \pm 0.021$ & $+0.207 \pm 0.030$ & $6.395 \pm 0.030$ \\
\hline 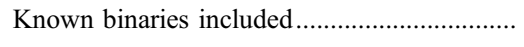 & $6.262 \pm 0.008$ & $6.279 \pm 0.008$ & $6.327 \pm 0.007$ & $6.293 \pm 0.020$ & $+0.186 \pm 0.030$ & $6.367 \pm 0.031$ \\
\hline 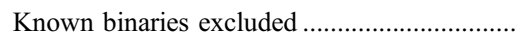 & $5.658 \pm 0.010$ & $5.623 \pm 0.013$ & $5.669 \pm 0.017$ & $5.649 \pm 0.013$ & $+0.021 \pm 0.032$ & $5.627 \pm 0.039$ \\
\hline 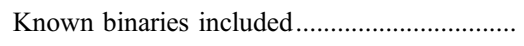 & $5.660 \pm 0.009$ & $5.639 \pm 0.013$ & $5.677 \pm 0.016$ & $5.657 \pm 0.009$ & $+0.035 \pm 0.028$ & $5.651 \pm 0.036$ \\
\hline \multicolumn{7}{|c|}{ M67 } \\
\hline $\mathrm{S} 04$ & $9.631 \pm 0.008$ & $9.601 \pm 0.007$ & $9.654 \pm 0.011$ & $9.622 \pm 0.015$ & $-0.002 \pm 0.062$ & $9.620 \pm 0.054$ \\
\hline MMJ93 & $9.630 \pm 0.010$ & $9.505 \pm 0.009$ & $9.626 \pm 0.008$ & $9.584 \pm 0.042$ & $-0.032 \pm 0.183$ & $9.562 \pm 0.163$ \\
\hline JTH01/RV+X-ray ${ }^{\mathrm{b}}$ & $8.115 \pm 0.010$ & $8.041 \pm 0.014$ & $8.077 \pm 0.021$ & $8.088 \pm 0.023$ & $-0.074 \pm 0.030$ & $7.995 \pm 0.037$ \\
\hline S02 & $8.098 \pm 0.014$ & $8.035 \pm 0.013$ & $8.064 \pm 0.012$ & $8.065 \pm 0.017$ & $-0.042 \pm 0.024$ & $8.015 \pm 0.026$ \\
\hline 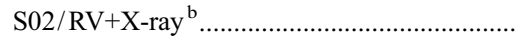 & $8.081 \pm 0.013$ & $7.960 \pm 0.022$ & $8.063 \pm 0.022$ & $8.052 \pm 0.034$ & $-0.059 \pm 0.081$ & $7.979 \pm 0.092$ \\
\hline
\end{tabular}

NotE.-All subsamples of data were filtered independently before fitting isochrones.

a Single cluster members from S04 with MMJ93 photometry.

b RV members from Terndrup et al. (2002) and X-ray-detected sources from Damiani et al. (2003).

stars, probably resulting from a paucity of Hyades stars with accurately measured parallaxes in this color range. In M67 (Fig. 8), there may be a larger deviation because the color calibration employed by $\mathrm{S} 04$ did not extend as far to the red as this.

The distance was independently derived from each CMD over the color range shown as a horizontal bar in each panel. The blue end of the color ranges for M67 was increased to $(B-V)_{0}=0.7$ to avoid the steeply rising parts of the MS, as the resulting distance would be sensitive to the choice of cluster age. For the Pleiades and NGC 2516, the red end of the color range in $(B-V$, $V)$ was decreased to $(B-V)_{0}=0.8$ to avoid systematic errors from the blue $\mathrm{K}$ dwarf phenomenon.

In Table 7 we assemble our derived cluster distances and the photometric metallicities. The first column lists the source of photometry, including particular selections of subsamples of the data. We applied the photometric filtering to each subsample before fitting isochrones. The second through fourth columns display the distance modulus found from each CMD, where the spectroscopic metallicity and reddening were taken from Tables 5 and 6, respectively. The weighted average distance, $(m-M)_{0, S}$, is shown in the fifth column. The last two columns display the photometric metallicity, $[\mathrm{M} / \mathrm{H}]_{E}$, and the distance modulus, $(m-M)_{0, E}$, at this metallicity. The error in $(m-M)_{0, S}$ is either the propagated one from the errors of the individual distances or the s.e.m. of these distance estimates, whichever is larger. The error in $(m-M)_{0, E}$ additionally includes the propagated error from $[\mathrm{M} / \mathrm{H}]_{E}$.

The standard errors of the average distance modulus at the spectroscopic metallicities are typically on the order of $0.02 \mathrm{mag}$ (i.e., the individual distances in the three CMDs are consistent to $\sim 2 \%$ ). In Figure 10 we show that this consistency is primarily the result of the empirical color- $T_{\text {eff }}$ corrections derived in Paper II. The top panel shows the derived distances to Praesepe as a function of the metallicity for isochrones that do not incorporate these corrections, while the bottom panel shows what happens

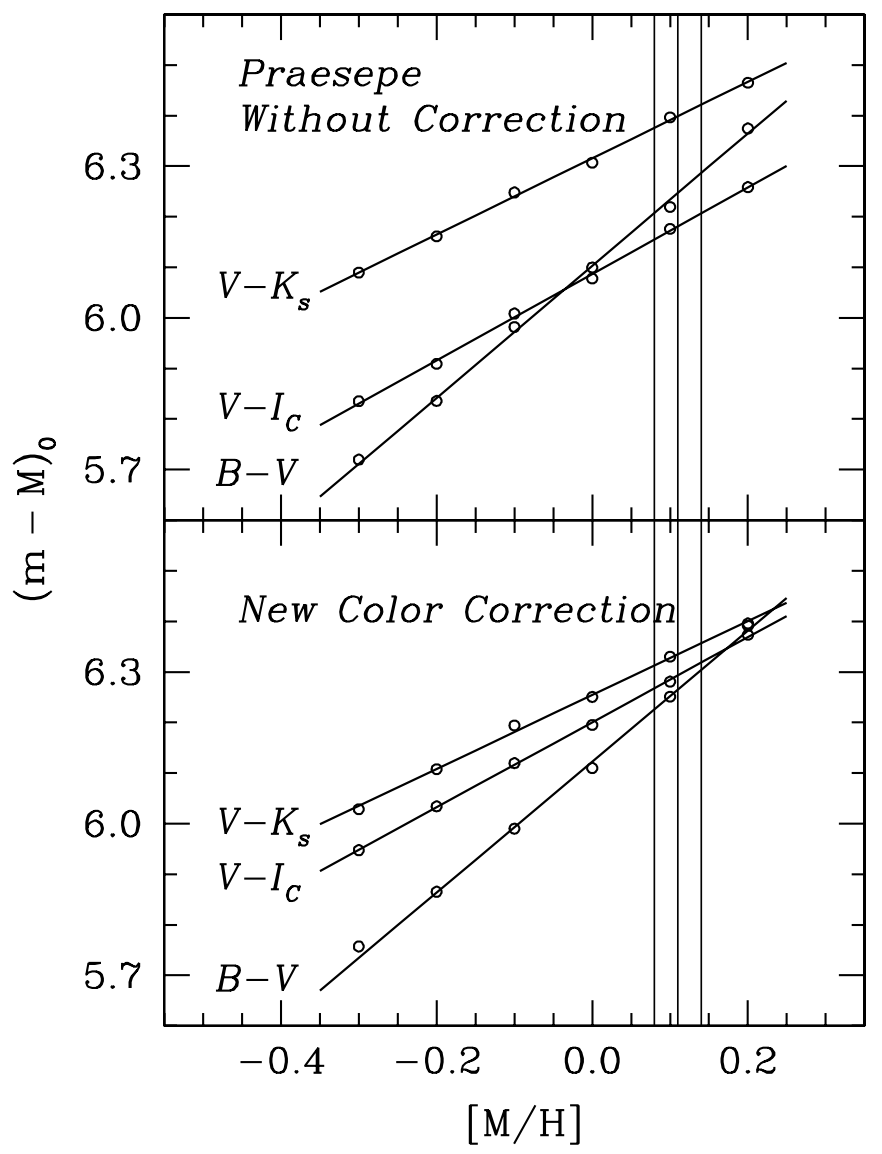

FIG. 10.-Distance vs. isochrone metallicity for Praesepe with the Lejeune et al. $(1997,1998)$ color- $T_{\text {eff }}$ relation (top) and with our new empirical correction (bottom). The reddening value in the literature (Table 6) was assumed. Circles and lines have the same meaning as in Fig. 5. Vertical lines show the spectroscopic metallicity and its $1 \sigma$ error (Table 5). 


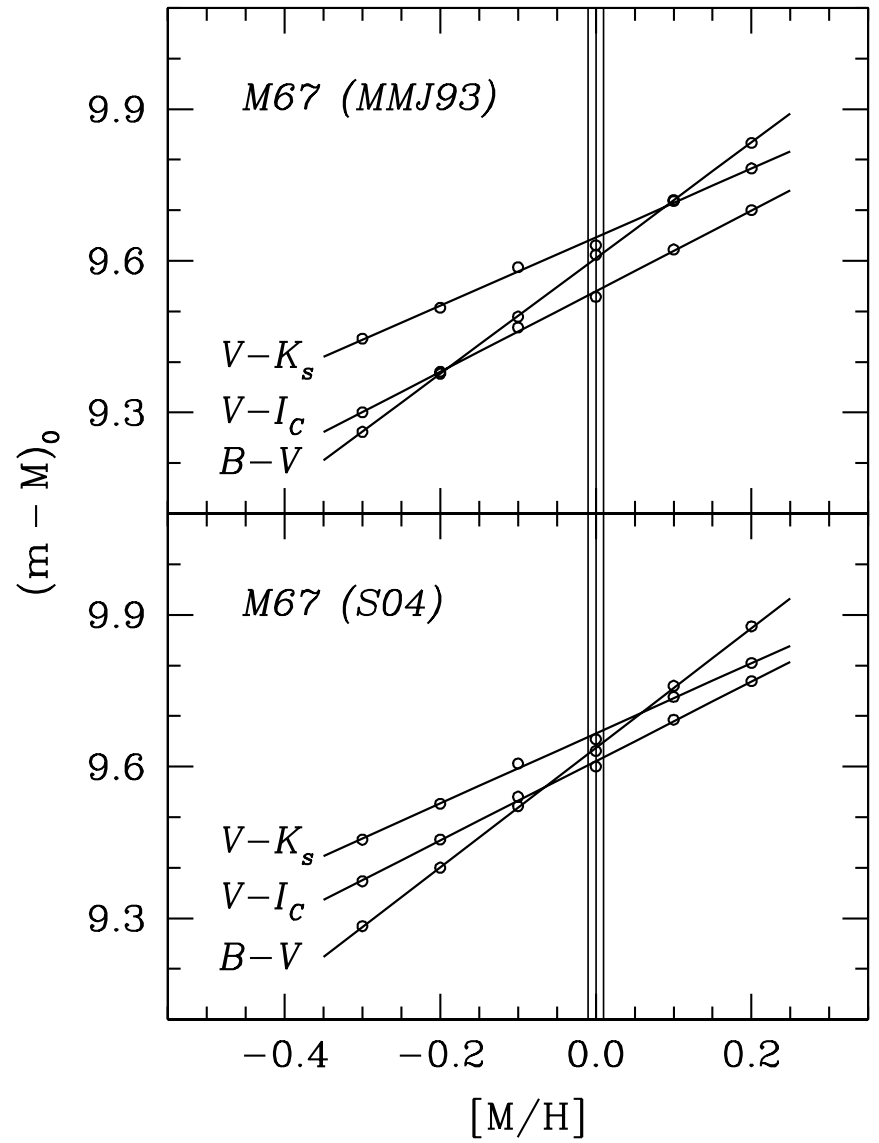

FIG. 11.- Same as Fig. 10, but for M67 with MMJ93 (top) and S04 (bottom) photometry. The stars designated as single cluster members by S04 were used in both cases.

when the corrected isochrones are employed. The vertical lines show the metallicity estimate for Praesepe with its $1 \sigma$ error $(\S 2.5 .1)$. The internal precision of the distance estimation with isochrones employing the Hyades empirical calibration is about a factor of 5 better than with the uncorrected isochrones.

The distances and photometric metallicities in Table 7 are generally not sensitive to the selection of subsamples in each cluster. For example, in Praesepe and the Pleiades, leaving known binaries in the sample before filtering changed the value of $(m-M)_{0, S}$ by only about $0.01 \mathrm{mag}$, confirming that the filtering algorithm removed most of the binaries that are brighter than the MS (e.g., Fig. 4). In M67, we compared the distances from the MMJ93 data, but also using their photometry for only those stars identified as single members by S04; we found a negligible difference. In NGC 2516, we computed the distances from the full JTH01 or S02 catalogs, or selecting only those stars identified as radial velocity (RV) members (Terndrup et al. 2002) or X-ray-detected sources (Damiani et al. 2003); again, making that selection reduced the distance modulus by only about $0.01 \mathrm{mag}$.

Figures 11-13 display the derived distances as a function of metallicity for M67, NGC 2516, and the Pleiades, respectively. The vertical lines on each plot show the average $[\mathrm{Fe} / \mathrm{H}]$ and its $1 \sigma$ error from Table 5. The distances for M67 (Fig. 11) are significantly less consistent with the MMJ93 photometry than from the S04 data. This is also seen in the larger errors in $[\mathrm{M} / \mathrm{H}]_{E}$ and $(m-M)_{0, E}$ in Table 7 . This is mainly because the $V-I_{\mathrm{C}}$ colors in MMJ93 are (on average) 0.022 mag redder than in S04, which

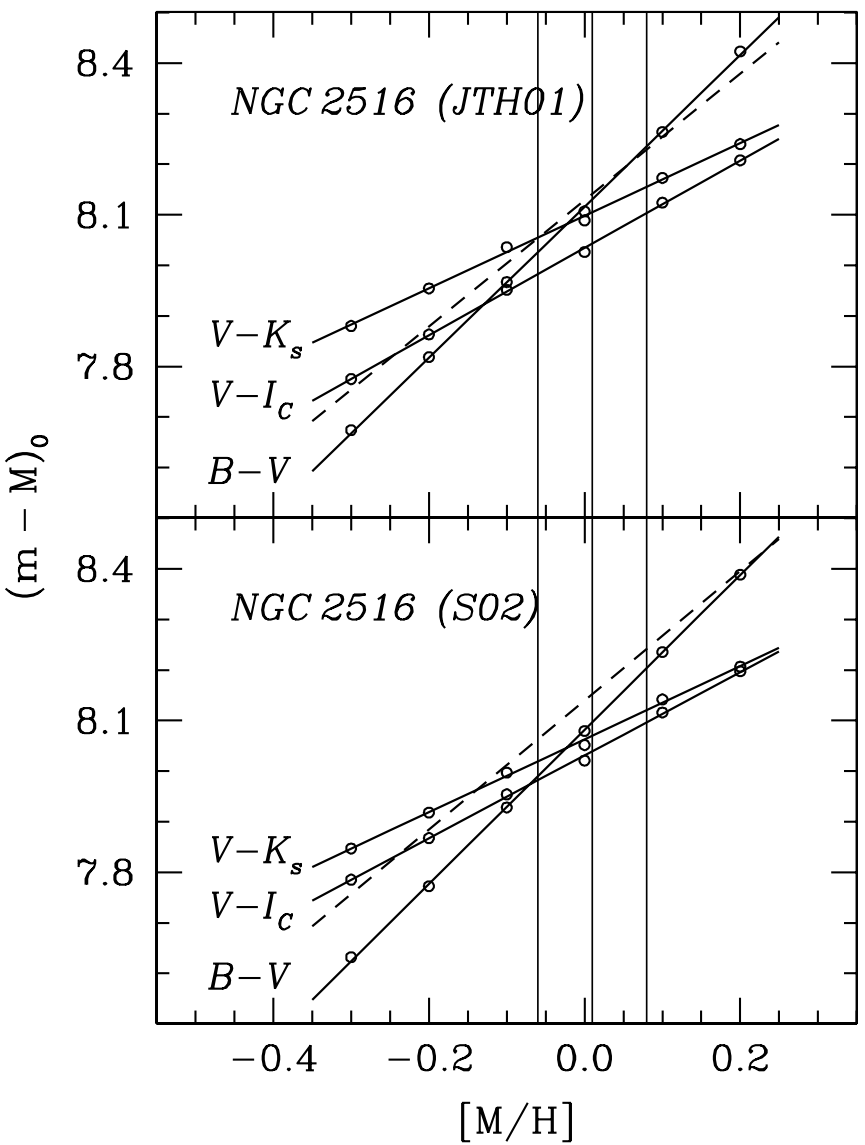

FIG. 12.- Same as Fig. 10, but for NGC 2516 with JTH01 (top) and S02 (bottom) photometry. The fitting range in $(B-V, V)$ was restricted to $0.4 \leq(B-V)_{0} \leq 0.8$ as in the case of the Pleiades. The dashed line shows the result when the full fitting range was used in this color.

will reduce the distance in the $\left(V-I_{\mathrm{C}}, V\right)$ by about $0.07 \mathrm{mag}$ since the average slope of the MS in this color is about 3.

In Figure 12 we explore the effects of the blue $\mathrm{K}$ dwarfs in NGC 2516 by comparing the two photometric data sets. The distances in $(B-V, V)$ derived over the truncated color range are shown as a solid line, while the dashed line displays the results for fits over $0.4 \leq(B-V)_{0} \leq 1.3$. The fits in the other two CMDs were done over the full color ranges. When the truncated color range is used, both JTH01 and S02 data give distances and photometric metallicities that are mutually consistent. However, fitting on the S02 data over the full range of colors in $B-V$ reduces the photometric metallicity by $\sim 0.12$ dex and the average distance at this metallicity by $\sim 0.09 \mathrm{mag}$, while the results from the JTH01 data remain consistent within $1 \sigma$ independently of the fitting range. We discuss the blue $\mathrm{K}$ dwarf phenomenon in NGC 2516 at greater length in $\S 7.1$.

In Figure 13 we display the fits for the Pleiades and also plot the average geometric distance and its $1 \sigma$ error from Table 1 as the horizontal lines. The agreement between the MS fitting and geometric distances is superb. Our distance for Praesepe at the spectroscopic metallicity also agrees with existing geometric distance determinations: Gatewood \& de Jonge (1994) found $(m-M)_{0}=6.42 \pm 0.33$ from ground-based parallaxes, and Loktin (2000) obtained $6.16 \pm 0.19$ from the moving cluster method. In addition, there are two determinations of the distance to Praesepe from Hipparcos: $(m-M)_{0}=6.37 \pm 0.15$ (van Leeuwen 1999) and 6.28 -0.12 (Robichon et al. 1999). We 


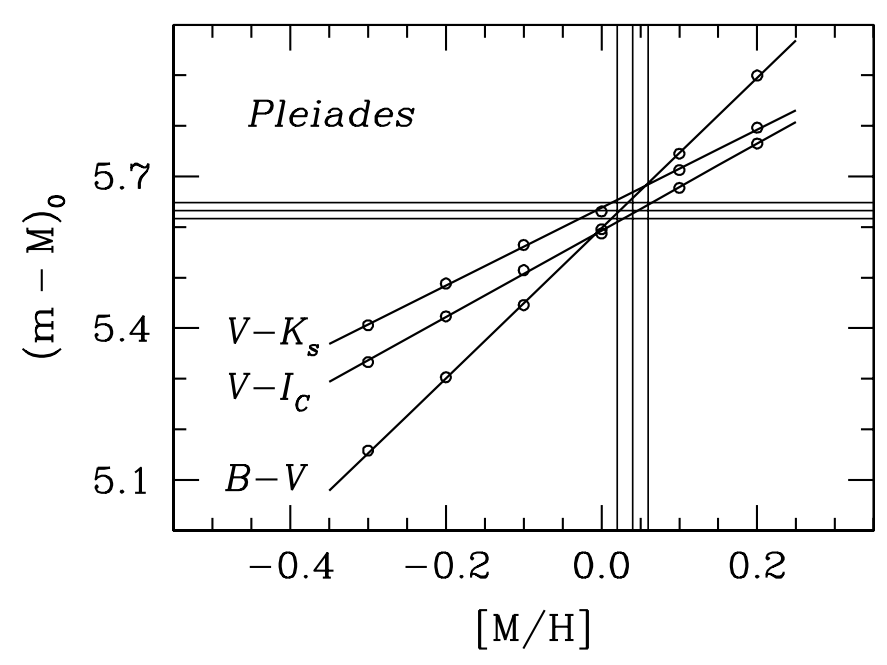

FIG. 13.-Same as Fig. 10, but for the Pleiades. Horizontal lines represent the average geometric distance and its $1 \sigma$ error from Table 1.

compare our derived distances with the Hipparcos measurements in $\S 7.2$.

\section{SIMULTANEOUS DETERMINATION OF CLUSTER PARAMETERS}

In the previous section we showed that the cluster metallicities can be obtained from the MS fitting because the various color indices have different sensitivities to the metallicity. However, we can further constrain the reddening because the slope of the MS becomes shallower below $M_{V} \approx 5.5$, especially in $\left(V-I_{\mathrm{C}}\right.$, $V)$ or $\left(V-K_{s}, V\right)$. Furthermore, the reddening vectors are not parallel to the MS as shown in Figure 6, so the derived distances depend on the reddening with different degrees of sensitivity in the three CMDs.

To determine the metallicity, reddening, and distance simultaneously, we minimized

$$
\chi_{\mathrm{tot}}^{2}=\sum_{j=1}^{3} \sum_{i=1}^{N_{j}} \frac{\left(\mu_{i j}-\bar{\mu}\right)^{2}}{\left[\sigma_{V, i j}^{2}+\left(\gamma_{i j} \sigma_{c, i j}\right)^{2}\right] f_{j}^{2}}
$$

where the same notation is used as in equation (1) except that the subscript $j$ is used to indicate each of the three CMDs. The $N_{j}$ is the number of data points used in the fit on the $j$ th CMD, and $\mu_{i j}$ are the individual distance moduli of the $i$ th point. The $\bar{\mu}$ is the weighted average distance modulus from the three CMDs.

The quantity $f_{j}$ in the denominator of equation (5) is a renormalization factor to take into account underestimation of the photometric errors. The value of $f_{j}$ was determined by comparing the residuals about the best-fitting isochrone at the minimum $\chi_{\text {tot }}^{2}$ (see below) to the propagated distance error from the photometric errors (eq. [4], $\left.\sigma_{\text {phot }}\right)$. This yields

$$
f_{j}=\max \left[\frac{\operatorname{MAD}\left(\mu_{i j}\right)}{\sqrt{N_{j}}} \frac{1}{\sigma_{\mathrm{phot}, j}}, 1\right] .
$$

This renormalization was required in particular for the S04 data, which had many more repeat measurements in $V$ and $I_{\mathrm{C}}$ than in $B$, so the quoted errors are much smaller in $V-I_{\mathrm{C}}$ than in the other colors. For these data, we found $f \approx 6$ in $\left(V-I_{\mathrm{C}}, V\right)$, indicating that the errors are greatly underestimated at least when compared to the scatter about the best-fitting isochrone. Without the renormalization factor, the majority of the weight in the $\chi_{\text {tot }}^{2}$ would be given to the $\left(V-I_{\mathrm{C}}, V\right) \mathrm{CMD}$, and the process would almost entirely ignore the information on the other CMDs.

We searched for the minimum $\chi_{\text {tot }}^{2}$ in the plane of $[\mathrm{M} / \mathrm{H}]$ versus $E(B-V)$ using a downhill simplex method (Press et al. 1992). The average distance modulus in equation (5) was determined at each $[\mathrm{M} / \mathrm{H}]$ and $E(B-V)$. Because the color ranges used in the fitting depend on the adopted $E(B-V)$, we solved for the minimum $\chi_{\text {tot }}^{2}$ at the $E(B-V)$ derived from the previous iteration to keep the same number of data points for each iteration. Since we defined $\chi_{\text {tot }}^{2}$ with respect to the average distance modulus from the three CMDs, the minimum $\chi_{\text {tot }}^{2}$ yields a set of $[\mathrm{M} / \mathrm{H}]$ and $E(B-V)$ that best describes all of the CMDs simultaneously.

Figure 14 shows likelihood contours in the $[\mathrm{M} / \mathrm{H}]$ versus $E(B-V)$ plane for all clusters in this paper and for the Hyades. The $\chi^{2}$ surfaces were smoothed with a Gaussian kernel. Contours are shown at $\Delta \chi_{\text {tot }}^{2}=3.53,8.02$, and 14.2 relative to the minimum value of $\chi_{\text {tot }}^{2}$, which correspond to $68.3 \%, 95.4 \%$, and $99.7 \%$ confidence levels $(1,2,3 \sigma)$ for the 3 degrees of freedom, and the errors in both quantities were assumed to be normally distributed without correlation. The vertical lines on each panel display the spectroscopic metallicity and its $1 \sigma$ error, while the horizontal lines show the average reddening in the literature with its $1 \sigma$ error. The error bars in each panel show the size of systematic errors, which is discussed in the next section.

The solution for Praesepe (Fig. 14, middle left panel) yields a value for the reddening that is slightly negative and a metallicity that is $\sim 2 \sigma$ higher than found spectroscopically. The negative reddening may be a consequence of zero-point errors in the photometry or else from an error in the Hyades reddening, which we assumed in Paper II as identically zero. The study of Taylor (1980), for example, indicates $E(B-V)=+0.003 \pm 0.002$ for the Hyades. So, if the Praesepe reddening was slightly less than the nonzero Hyades reddening, the MS fitting method would yield a negative value.

In Figure 14 two solutions are shown for M67 (top middle panel): the contours with the higher $E(B-V)$ are from the MMJ93 data, and the others are from the S04. The large difference in $E(B-V)$ obtained from these two samples is primarily due to the difference in the $V-I_{\mathrm{C}}$ values (Table 2). We illustrate this further by showing the $\chi^{2}$ contours that result when only the $B-V$ and $V-K_{s}$ data are included. The middle panel shows the likelihood contours for the MMJ93 data, while the bottom middle panel is for the S04. Here the two studies yield the same values of metallicity and reddening, although with larger errors. We also display two solutions for NGC 2516 in the bottom left panel of Figure 14. The contours with the higher reddening are from S02, and the others are from the JTH01 photometry.

The top right panel of Figure 14 shows the solution for the Pleiades, while the panel below that shows the solution for the metallicity and reddening that result if the average geometric distance from Table 1 is taken as a prior. Contours in this panel are 1,2 , and $3 \sigma$ confidence levels for the 2 degrees of freedom. This demonstrates that good photometry and parallaxes together can provide strong constraints on the metal abundance of a cluster.

Table 8 lists the results from the $\chi^{2}$ solutions for various combinations of the data listed in the first column. The $[\mathrm{M} / \mathrm{H}]_{\chi^{2}}$ and $E(B-V)_{\chi^{2}}$ denote our derived values of metallicity and reddening, respectively, and are shown in the third and fourth columns. Errors in these quantities are the sizes of the $1 \sigma$ contours in Figure 14 . The $(m-M)_{0, \chi^{2}}$ in the second column is the average distance from the three CMDs. The errors in metallicity 

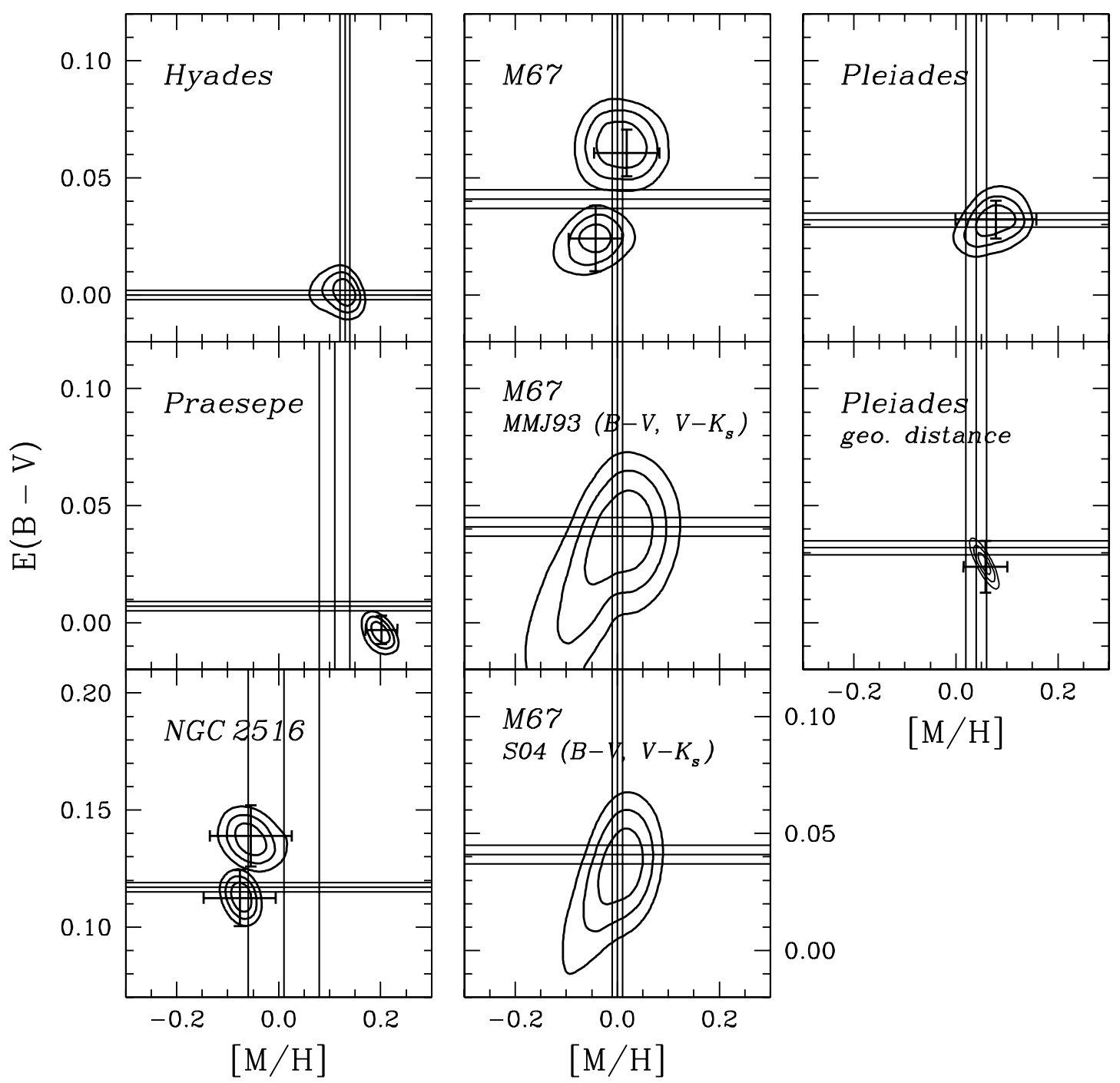

FIG. 14.- Likelihood contours in [M/H] vs. $E(B-V)$ shown at $\Delta \chi_{\text {tot }}^{2}=3.53,8.02$, and $14.2(1,2$, and $3 \sigma$ for 3 degrees of freedom) relative to the minimum value of $\chi_{\text {tot }}^{2}$ (eq. [5]). Those for 2 degrees of freedom are shown for the Pleiades at the geometric distance (middle right). For NGC 2516 (bottom left), contours with the higher $E(B-V)$ are from the S02, and the others are from the JTH01 photometry. For M67 (top middle), contours with the higher $E(B-V)$ are from the MMJ93, and the others are from the S04 photometry. Also shown are contours for the MMJ93 (middle) and the S04 (bottom middle) in the case where only $(B-V, V)$ and $\left(V-K_{s}, V\right)$ are used. Error bars represent $1 \sigma$ systematic errors listed in Tables 9-12 excluding fitting errors. The spectroscopic metallicities and reddening values in the literature with $1 \sigma$ errors (Tables 5 and 6) are shown with vertical and horizontal lines, respectively.

and reddening were propagated into the error in distance. We compare these values with previous estimates in the literature after a discussion of systematic errors in the next section.

\section{SYSTEMATIC ERRORS AND THE ACCURACY OF MS FITTING}

In the previous two sections we showed that MS fitting can be performed with high internal precision, resulting in errors in distance moduli of $0.02 \mathrm{mag}$ (i.e., $1 \%$ in distance) at the spectroscopic metallicity (Table 7). When spectroscopy is not available, photometry in $B V I_{\mathrm{C}} K_{s}$ alone can be used to derive distances with precision of $1 \%-3 \%$, metallicities with $0.02-0.04$ dex error, and reddening estimates with better than 0.01 mag error (Table 8). However, various systematic errors in the MS fitting should be considered in the final error budget such as the photometric zero-point errors or biases introduced by the cluster binaries and foreground/background stars. In this section we estimate these systematic errors and show that they often exceed the internal precision of the MS fitting by factors of 2-3.

\subsection{Errors from Input Quantities}

In Tables 9-12 we list the sources of several systematic errors and their contributions to errors in the distance, photometric metallicity, and reddening estimates for each cluster. The first column displays the source of errors, and the second column shows the size of the errors adopted for each cluster. The third column lists error contributions to $(m-M)_{0, S}$ when both metallicity and reddening are held fixed. The fourth and fifth columns contain errors in $(m-M)_{0, E}$ and $[\mathrm{M} / \mathrm{H}]_{E}$ when the photometric metallicity is determined at a fixed reddening. The sixth through eighth columns list errors in $(m-M)_{0, \chi^{2}},[\mathrm{M} / \mathrm{H}]_{\chi^{2}}$, and $E(B-V)_{\chi^{2}}$ from the $\chi^{2}$ minimization. At the bottom of the table, we list the "total error," computed as the quadrature sum of all of the error contributions. These are the errors that are shown as error bars along with the $\chi^{2}$ contours in Figure 14. The fitting errors (i.e., the internal precision of MS fitting) are not included in these sums but instead are separately tabulated below.

In the first column of these tables, the line marked $[\mathrm{Fe} / \mathrm{H}] \mathrm{de}-$ notes the error in the adopted cluster metallicity (Table 5). This 
TABLE 8

$\chi^{2}$ Solutions

\begin{tabular}{|c|c|c|c|}
\hline Data/Constraint & $(m-M)_{0, \chi^{2}}$ & {$[\mathrm{M} / \mathrm{H}]_{\chi^{2}}$} & $E(B-V)_{\chi^{2}}$ \\
\hline \multicolumn{4}{|c|}{ Praesepe } \\
\hline Known binaries excluded .............................. & $6.365 \pm 0.025$ & $+0.202 \pm 0.020$ & $-0.003 \pm 0.005$ \\
\hline Known binaries included .................................. & $6.357 \pm 0.026$ & $+0.192 \pm 0.020$ & $0.002 \pm 0.004$ \\
\hline \multicolumn{4}{|c|}{ Pleiades } \\
\hline Known binaries excluded .......................................... & $5.693 \pm 0.053$ & $+0.079 \pm 0.040$ & $0.032 \pm 0.007$ \\
\hline 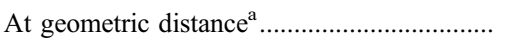 & $5.632 \pm 0.017^{\mathrm{b}}$ & $+0.058 \pm 0.012$ & $0.024 \pm 0.004$ \\
\hline 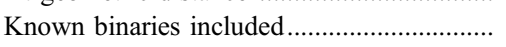 & $5.667 \pm 0.054$ & $+0.059 \pm 0.040$ & $0.027 \pm 0.007$ \\
\hline 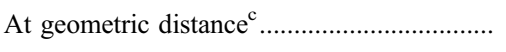 & $5.632 \pm 0.017^{\mathrm{b}}$ & $+0.049 \pm 0.012$ & $0.025 \pm 0.004$ \\
\hline \multicolumn{4}{|c|}{ M67 } \\
\hline $\mathrm{S} 04$ & $9.558 \pm 0.034$ & $-0.043 \pm 0.032$ & $0.024 \pm 0.006$ \\
\hline 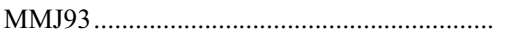 & $9.603 \pm 0.051$ & $-0.061 \pm 0.035$ & $0.078 \pm 0.006$ \\
\hline MMJ93/S04 stars ${ }^{\mathrm{d}}$ & $9.638 \pm 0.053$ & $+0.019 \pm 0.050$ & $0.061 \pm 0.010$ \\
\hline \multicolumn{4}{|c|}{ NGC 2516} \\
\hline 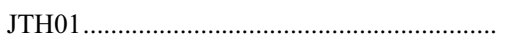 & $8.001 \pm 0.038$ & $-0.076 \pm 0.020$ & $0.112 \pm 0.007$ \\
\hline 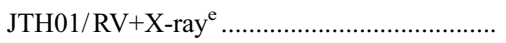 & $7.939 \pm 0.059$ & $-0.133 \pm 0.040$ & $0.120 \pm 0.010$ \\
\hline $\mathrm{S} 02$ & $8.064 \pm 0.038$ & $-0.055 \pm 0.030$ & $0.139 \pm 0.007$ \\
\hline S02 / RV+X-ray ${ }^{\mathrm{e}}$ & $8.038 \pm 0.091$ & $-0.050 \pm 0.060$ & $0.134 \pm 0.015$ \\
\hline
\end{tabular}

NoTE.-All subsamples of data were filtered independently before fitting isochrones.

${ }^{a}$ Solution at the average geometric distance after excluding known binaries.

b Average geometric distance from Table 1 .

${ }^{c}$ Solution at the average geometric distance with known binaries included in the data

d Single cluster members from S04 with MMJ93 photometry.

e RV members from Terndrup et al. (2002) and X-ray-detected sources from Damiani et al. (2003).

affects the error in $(m-M)_{0, S}$ only. The errors in the adopted reddening (Table 6) are shown in the line marked $E(B-V)$. These errors are propagated into the determination of the photometric metallicity $[\mathrm{M} / \mathrm{H}]_{E}$ and the distance $(m-M)_{0, E}$, but not the values determined from the $\chi^{2}$ minimization. The remaining errors will contribute to the systematic errors of all of the parameters found via MS fitting.

We chose an error of $30 \%$ in the adopted age of each cluster from the consideration of previous age estimates for the Pleiades. The cluster age is about 100 Myr from the MS turnoff using isochrones that incorporate convective overshooting (Meynet et al. 1993), while ages from the lithium depletion boundary are about $125 \mathrm{Myr}$ (Stauffer et al. 1998; Burke et al. 2004). However, distances, photometric metallicities, and reddening values are insensitive to age because the color range we chose for the fitting avoids the upper MS and the pre-MS (for clusters at least as old as the Pleiades).

The helium abundance $(Y)$ sensitively affects the isochrone luminosity $\left(\Delta M_{V} / \Delta Y \approx 3\right.$ at fixed $\left.T_{\text {eff }}\right)$. A shorter distance is obtained when a higher helium abundance is assumed. We adopted

TABLE 9

MS Fitting Error Budget for Praesepe

\begin{tabular}{|c|c|c|c|c|c|c|c|}
\hline \multirow[b]{2}{*}{ SOURCE OF ERror } & \multirow[b]{2}{*}{$\Delta$ QUANTITY } & \multirow{2}{*}{$\begin{array}{c}\text { AdOPTED }[\mathrm{Fe} / \mathrm{H}] \\
\Delta(m-M)_{0, S}\end{array}$} & \multicolumn{2}{|c|}{ Adopted $E(B-V)$} & \multicolumn{3}{|c|}{$\chi^{2}$ Minimization } \\
\hline & & & $\Delta(m-M)_{0, E}$ & {$[\mathrm{M} / \mathrm{H}]_{E}$} & $\Delta(m-M)_{0, \chi^{2}}$ & {$[\mathrm{M} / \mathrm{H}]_{\chi^{2}}$} & $E(B-V)_{\chi^{2}}$ \\
\hline$[\mathrm{Fe} / \mathrm{H}]$ & \pm 0.03 & \pm 0.029 & & & $\ldots$ & $\ldots$ & $\ldots$ \\
\hline$E(B-V)$ & \pm 0.002 & \pm 0.005 & \pm 0.013 & \pm 0.007 & $\ldots$ & $\ldots$ & $\ldots$ \\
\hline 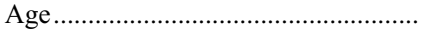 & $\pm 30 \%$ & \pm 0.007 & \pm 0.010 & \pm 0.000 & $\mp 0.014$ & $\mp 0.013$ & $\mp 0.004$ \\
\hline Helium $(Y)$ & \pm 0.009 & $\mp 0.027$ & $\mp 0.027$ & \pm 0.000 & $\mp 0.027$ & \pm 0.000 & \pm 0.000 \\
\hline 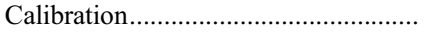 & $\ldots$ & \pm 0.010 & \pm 0.020 & \pm 0.020 & \pm 0.010 & \pm 0.010 & \pm 0.002 \\
\hline 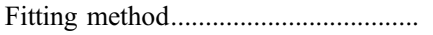 & $\ldots$ & \pm 0.006 & \pm 0.014 & \pm 0.013 & \pm 0.002 & \pm 0.004 & \pm 0.001 \\
\hline$R_{V}$ & \pm 0.3 & \pm 0.000 & \pm 0.006 & \pm 0.006 & \pm 0.000 & $\mp 0.001$ & \pm 0.000 \\
\hline$R_{V I}$ & \pm 0.07 & \pm 0.001 & \pm 0.004 & \pm 0.003 & $\mp 0.002$ & $\mp 0.003$ & \pm 0.001 \\
\hline$R_{V K}$ & \pm 0.12 & \pm 0.001 & \pm 0.002 & \pm 0.001 & \pm 0.000 & \pm 0.000 & \pm 0.000 \\
\hline$\Delta V$ & \pm 0.006 & \pm 0.001 & $\mp 0.011$ & $\mp 0.011$ & $\mp 0.003$ & $\mp 0.008$ & \pm 0.002 \\
\hline$\Delta K_{s}$ & \pm 0.007 & \pm 0.006 & \pm 0.019 & \pm 0.013 & \pm 0.010 & \pm 0.010 & $\mp 0.002$ \\
\hline$\Delta(B-V)$ & \pm 0.004 & $\mp 0.006$ & \pm 0.038 & \pm 0.044 & \pm 0.014 & \pm 0.027 & $\mp 0.002$ \\
\hline 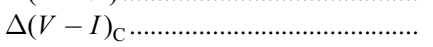 & \pm 0.006 & $\mp 0.008$ & $\mp 0.027$ & $\mp 0.021$ & $\mp 0.007$ & $\mp 0.006$ & \pm 0.002 \\
\hline 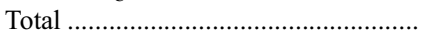 & $\ldots$ & \pm 0.044 & \pm 0.065 & \pm 0.058 & \pm 0.037 & \pm 0.035 & \pm 0.006 \\
\hline 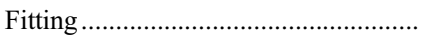 & $\ldots$ & \pm 0.021 & \pm 0.030 & \pm 0.030 & \pm 0.025 & \pm 0.020 & \pm 0.005 \\
\hline
\end{tabular}


TABLE 10

MS Fitting Error Budget for the Pleiades

\begin{tabular}{|c|c|c|c|c|c|c|c|}
\hline \multirow[b]{2}{*}{ SOURCE OF ERror } & \multirow[b]{2}{*}{$\Delta$ QUANTITY } & \multirow{2}{*}{$\begin{array}{c}\text { AdOPTED [Fe/H] } \\
\Delta(m-M)_{0, S}\end{array}$} & \multicolumn{2}{|c|}{ Adopted $E(B-V)$} & \multicolumn{3}{|c|}{$\chi^{2}$ Minimization } \\
\hline & & & $\Delta(m-M)_{0, E}$ & {$[\mathrm{M} / \mathrm{H}]_{E}$} & $\Delta(m-M)_{0, \chi^{2}}$ & {$[\mathrm{M} / \mathrm{H}]_{\chi^{2}}$} & $E(B-V)_{\chi^{2}}$ \\
\hline 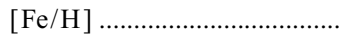 & \pm 0.02 & \pm 0.023 & $\ldots$ & $\ldots$ & $\ldots$ & $\ldots$ & $\ldots$ \\
\hline 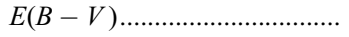 & \pm 0.003 & \pm 0.007 & \pm 0.010 & \pm 0.002 & $\ldots$ & $\ldots$ & $\ldots$ \\
\hline Age & $\pm 30 \%$ & +0.006 & +0.020 & +0.011 & +0.004 & +0.002 & +0.001 \\
\hline 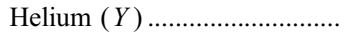 & \pm 0.009 & $\mp 0.027$ & $\mp 0.027$ & \pm 0.000 & $\mp 0.027$ & \pm 0.000 & \pm 0.000 \\
\hline Calibration............. & $\ldots$ & \pm 0.010 & \pm 0.020 & \pm 0.020 & \pm 0.010 & \pm 0.010 & \pm 0.002 \\
\hline 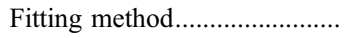 & $\ldots$ & \pm 0.006 & \pm 0.038 & \pm 0.033 & \pm 0.027 & \pm 0.014 & \pm 0.005 \\
\hline 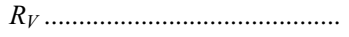 & \pm 0.3 & $\mp 0.003$ & \pm 0.018 & \pm 0.018 & \pm 0.013 & \pm 0.016 & $\mp 0.001$ \\
\hline 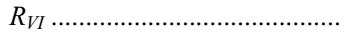 & \pm 0.07 & \pm 0.003 & \pm 0.012 & \pm 0.008 & \pm 0.010 & \pm 0.008 & $\mp 0.001$ \\
\hline$R_{V K}$ & \pm 0.12 & \pm 0.002 & \pm 0.008 & \pm 0.005 & \pm 0.007 & \pm 0.005 & \pm 0.000 \\
\hline$\Delta V$ & \pm 0.012 & \pm 0.008 & $\mp 0.009$ & $\mp 0.014$ & $\mp 0.013$ & $\mp 0.018$ & \pm 0.000 \\
\hline$\Delta K_{s} \ldots \ldots \ldots \ldots \ldots \ldots \ldots \ldots \ldots \ldots \ldots \ldots \ldots \ldots \ldots \ldots \ldots \ldots$ & \pm 0.007 & \pm 0.003 & \pm 0.012 & \pm 0.008 & \pm 0.014 & \pm 0.010 & \pm 0.000 \\
\hline$\Delta(B-V) \ldots \ldots \ldots \ldots \ldots \ldots \ldots \ldots \ldots \ldots \ldots \ldots \ldots \ldots \ldots$ & \pm 0.008 & $\mp 0.023$ & \pm 0.058 & \pm 0.069 & \pm 0.048 & \pm 0.066 & $\mp 0.002$ \\
\hline$\Delta(V-I)_{\mathrm{C}} \ldots \ldots \ldots \ldots \ldots \ldots \ldots \ldots \ldots \ldots \ldots \ldots \ldots \ldots$ & \pm 0.009 & $\mp 0.013$ & $\mp 0.037$ & $\mp 0.021$ & $\mp 0.034$ & $\mp 0.030$ & \pm 0.006 \\
\hline 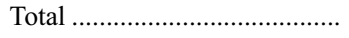 & $\ldots$ & \pm 0.048 & \pm 0.092 & \pm 0.087 & \pm 0.076 & \pm 0.080 & \pm 0.009 \\
\hline 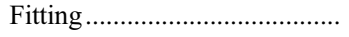 & $\ldots$ & \pm 0.013 & \pm 0.039 & \pm 0.032 & \pm 0.053 & \pm 0.040 & \pm 0.007 \\
\hline
\end{tabular}

an error in $Y$ of 0.009 for each cluster. This is a $1 \sigma$ scatter in $\Delta Y / \Delta Z$ from the primordial helium abundance, the solar value, and that of the Hyades as inferred from the MS luminosity at the Hipparcos distance (see Paper I). The photometric metallicities and reddening values are not affected by the helium abundance since the error in Yequally changes the distances in the three CMDs.

The calibration errors in the tables reflect errors in our adopted parameters of the Hyades: $[\mathrm{Fe} / \mathrm{H}]=+0.13 \pm 0.01$ (Paulson et al. 2003) and the center-of-mass distance modulus of $(m-M)_{0}=$ $3.33 \pm 0.01$ (Perryman et al. 1998). We adopted a $1 \sigma$ error in reddening from Taylor (1980), $E(B-V)=+0.003 \pm 0.002$, but assumed a zero reddening toward the Hyades (Paper II). The errors in $[\mathrm{M} / \mathrm{H}]_{E}$ and $(m-M)_{0, E}$ further include the errors arising from the scatter about the linear relation between the two quantities (see Fig. 5). The error from the fitting method comes from experiments in which we used the weighted mean or weighted median distance modulus instead of the unweighted median value.

The next three rows in the tables show the effects of errors in the reddening laws. Wegner (2003) determined $R_{V}$ toward about $600 \mathrm{OB}$ stars, from which we estimated an uncertainty of \pm 0.3 from the MAD of the distribution. We estimated the dependence of $R_{V I}$ and $R_{V K}$ on the choice of $R_{V}$ from the extinction law of Cardelli et al. (1989) and found $\Delta R_{V I} / \Delta R_{V} \simeq 0.22$ and $\Delta R_{V K} / \Delta R_{V} \simeq 0.84$ for B-type stars. We adopted \pm 0.07 for the error in $R_{V I}$ from the difference between our adopted value and $R_{V I}=1.24$ from Dean et al. (1978). Similarly, we adopted \pm 0.12 as the error in $R_{V K}$ by considering our value with those advocated by Schultz \& Wiemer (1975) and by Sneden et al. (1978). These are significant contributors to the error budget for the highly reddened cluster (NGC 2516) but are much less significant for the other clusters.

The next four rows list the errors resulting from zero-point errors in the photometry. In this calculation, we assumed that the fundamental observed quantities are $V, K_{s}, B-V$, and $V-I_{\mathrm{C}}$ (e.g., Stetson et al. 2003). The adopted values of the errors are shown in the second column. The error in $K_{s}$ was taken as the calibration uncertainty $(0.007 \mathrm{mag})$ that was specified in the explanatory supplement to the 2MASS All Sky Data Release. ${ }^{12}$ For the Pleiades and Praesepe, the errors come from intercomparisons between limited subsets of stars in common among various

12 See http://www.ipac.caltech.edu/2mass/releases/allsky/doc/explsup.html.

TABLE 11

MS Fitting ERror Budget for M67

\begin{tabular}{|c|c|c|c|c|c|c|c|}
\hline \multirow[b]{2}{*}{ SOURCE OF ERROR } & \multirow[b]{2}{*}{$\Delta$ QUANTITY } & \multirow{2}{*}{$\begin{array}{c}\text { Adopted }[\mathrm{Fe} / \mathrm{H}] \\
\Delta(m-M)_{0, S}\end{array}$} & \multicolumn{2}{|c|}{ AdOPtED $E(B-V)$} & \multicolumn{3}{|c|}{$\chi^{2}$ Minimization } \\
\hline & & & $\Delta(m-M)_{0, E}$ & {$[\mathrm{M} / \mathrm{H}]_{E}$} & $\Delta(m-M)_{0, \chi^{2}}$ & {$[\mathrm{M} / \mathrm{H}]_{\chi^{2}}$} & $E(B-V)_{\chi^{2}}$ \\
\hline$[\mathrm{Fe} / \mathrm{H}]$ & \pm 0.01 & \pm 0.008 & & & $\ldots$ & $\ldots$ & $\ldots$ \\
\hline$E(B-V)$ & \pm 0.004 & \pm 0.008 & \pm 0.016 & \pm 0.009 & $\ldots$ & & $\ldots$ \\
\hline 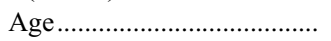 & $\pm 30 \%$ & \pm 0.006 & \pm 0.009 & \pm 0.005 & \pm 0.001 & $\mp 0.002$ & $\mp 0.002$ \\
\hline Helium $(Y)$ & \pm 0.009 & $\mp 0.027$ & $\mp 0.027$ & \pm 0.000 & $\mp 0.027$ & \pm 0.000 & \pm 0.000 \\
\hline 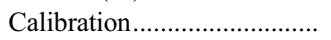 & $\ldots$ & \pm 0.010 & \pm 0.020 & \pm 0.020 & \pm 0.010 & \pm 0.010 & \pm 0.002 \\
\hline 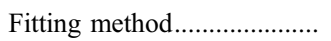 & $\ldots$ & \pm 0.004 & \pm 0.011 & \pm 0.016 & \pm 0.001 & \pm 0.001 & \pm 0.000 \\
\hline$R_{V}$ & \pm 0.3 & $\mp 0.004$ & \pm 0.019 & \pm 0.026 & \pm 0.000 & \pm 0.008 & $\mp 0.002$ \\
\hline$R_{V I}$ & \pm 0.07 & \pm 0.006 & \pm 0.014 & \pm 0.010 & \pm 0.004 & \pm 0.002 & $\mp 0.001$ \\
\hline$R_{V K}$ & \pm 0.12 & \pm 0.001 & \pm 0.007 & \pm 0.007 & \pm 0.002 & \pm 0.001 & \pm 0.000 \\
\hline$\Delta V$ & \pm 0.009 & \pm 0.006 & $\mp 0.006$ & $\mp 0.014$ & $\mp 0.007$ & $\mp 0.016$ & \pm 0.000 \\
\hline$\Delta K_{s}$ & \pm 0.007 & \pm 0.002 & \pm 0.011 & \pm 0.011 & \pm 0.012 & \pm 0.012 & \pm 0.000 \\
\hline$\Delta(B-V)$ & \pm 0.005 & $\mp 0.006$ & \pm 0.038 & \pm 0.049 & \pm 0.026 & \pm 0.043 & $\mp 0.003$ \\
\hline$\Delta(V-I)_{\mathrm{C}} \cdots \ldots \ldots$ & \pm 0.011 & $\mp 0.022$ & $\mp 0.045$ & $\mp 0.023$ & $\mp 0.003$ & $\mp 0.009$ & \pm 0.014 \\
\hline 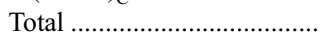 & $\ldots$ & \pm 0.040 & \pm 0.076 & \pm 0.070 & \pm 0.041 & \pm 0.050 & \pm 0.014 \\
\hline 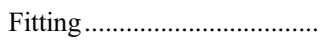 & $\ldots$ & \pm 0.015 & \pm 0.054 & \pm 0.062 & \pm 0.034 & \pm 0.032 & \pm 0.006 \\
\hline
\end{tabular}

NotE.-Estimated from S04 photometry. 
TABLE 12

MS Fitting Error Budget for NGC 2516

\begin{tabular}{|c|c|c|c|c|c|c|c|}
\hline \multirow[b]{2}{*}{ SOURCE OF ERROR } & \multirow[b]{2}{*}{$\Delta$ QUANTITY } & \multirow{2}{*}{$\begin{array}{c}\text { AdOPTED }[\mathrm{Fe} / \mathrm{H}] \\
\Delta(m-M)_{0, S}\end{array}$} & \multicolumn{2}{|c|}{ Adopted $E(B-V)$} & \multicolumn{3}{|c|}{$\chi^{2}$ Minimization } \\
\hline & & & $\Delta(m-M)_{0, E}$ & {$[\mathrm{M} / \mathrm{H}]_{E}$} & $\Delta(m-M)_{0, \chi^{2}}$ & {$[\mathrm{M} / \mathrm{H}]_{\chi^{2}}$} & $E(B-V)_{\chi^{2}}$ \\
\hline$[\mathrm{Fe} / \mathrm{H}]$ & \pm 0.07 & \pm 0.072 & $\ldots$ & $\ldots$ & $\ldots$ & $\ldots$ & $\ldots$ \\
\hline$E(B-V) \ldots \ldots \ldots \ldots \ldots \ldots \ldots \ldots \ldots \ldots \ldots \ldots \ldots \ldots \ldots \ldots$ & \pm 0.002 & \pm 0.006 & \pm 0.005 & \pm 0.000 & $\ldots$ & $\ldots$ & $\ldots$ \\
\hline Age & $\pm 30 \%$ & \pm 0.005 & \pm 0.014 & \pm 0.008 & \pm 0.014 & \pm 0.006 & \pm 0.000 \\
\hline 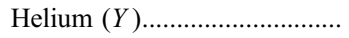 & \pm 0.009 & $\mp 0.027$ & $\mp 0.027$ & \pm 0.000 & $\mp 0.027$ & \pm 0.000 & \pm 0.000 \\
\hline 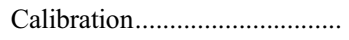 & $\ldots$ & \pm 0.010 & \pm 0.020 & \pm 0.020 & \pm 0.010 & \pm 0.010 & \pm 0.002 \\
\hline 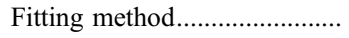 & $\ldots$ & \pm 0.009 & \pm 0.016 & \pm 0.019 & \pm 0.002 & \pm 0.003 & \pm 0.000 \\
\hline 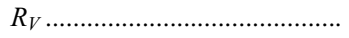 & \pm 0.3 & $\mp 0.009$ & \pm 0.051 & \pm 0.058 & \pm 0.030 & \pm 0.063 & $\mp 0.010$ \\
\hline 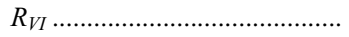 & \pm 0.07 & \pm 0.008 & \pm 0.017 & \pm 0.009 & \pm 0.011 & \pm 0.005 & \pm 0.000 \\
\hline 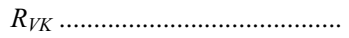 & \pm 0.12 & \pm 0.009 & \pm 0.031 & \pm 0.023 & \pm 0.016 & \pm 0.021 & $\mp 0.005$ \\
\hline$\Delta V$ & \pm 0.008 & \pm 0.002 & $\mp 0.011$ & $\mp 0.015$ & $\mp 0.004$ & $\mp 0.013$ & \pm 0.002 \\
\hline 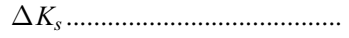 & \pm 0.007 & \pm 0.005 & \pm 0.017 & \pm 0.013 & \pm 0.011 & \pm 0.012 & $\mp 0.002$ \\
\hline$\Delta(B-V)$ & \pm 0.002 & $\mp 0.004$ & \pm 0.012 & \pm 0.016 & \pm 0.011 & \pm 0.016 & \pm 0.000 \\
\hline$\Delta(V-I)_{\mathrm{C}} \cdots \cdots \cdots \cdots \cdots \cdots \cdots \cdots \cdots \cdots \cdots \cdots \cdots \cdots$ & \pm 0.006 & $\mp 0.007$ & $\mp 0.014$ & $\mp 0.007$ & $\mp 0.009$ & $\mp 0.007$ & \pm 0.002 \\
\hline 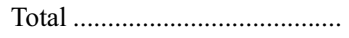 & $\ldots$ & \pm 0.080 & \pm 0.079 & \pm 0.074 & \pm 0.051 & \pm 0.072 & \pm 0.012 \\
\hline 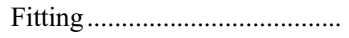 & $\ldots$ & \pm 0.023 & \pm 0.058 & \pm 0.053 & \pm 0.038 & \pm 0.020 & \pm 0.007 \\
\hline
\end{tabular}

NoTE.-Estimated from JTH01 photometry.

studies and from a consideration of the entries in Tables 2 and 3. For M67 and NGC 2516, we assumed the magnitude and color errors to be half the difference between the two studies (Table 2).

Besides the systematic errors in the photometry, distance estimation can be affected by the variability of pre-MS and MS stars (e.g., Scholz \& Eislöffel 2004), which may be caused by rotational modulation of cool and hot regions on stellar surfaces. This issue can be addressed with multiple observations for each star, but those kinds of data are not usually available.

Errors in the metallicity and the helium abundance are important contributors to the error in the distance $(m-M)_{0, S}$. As shown in Table 5, individual spectroscopic studies generally have $\sim 0.03$ dex errors in the mean metal abundance, and this is propagated into the error of $\sim 0.03 \mathrm{mag}$ in distance modulus since $\Delta(m-M)_{0} / \Delta[\mathrm{Fe} / \mathrm{H}] \approx 1$. In M67, the error in the metallicity has no greater effect than the other systematic errors due to the many independent metallicity estimates. This contrasts well with the NGC 2516 case where only two stars were measured from high-resolution spectroscopy. The zero-point errors in the photometry dominate the systematic errors in the photometric metallicity and the distance derived purely from the isochrones, independently of the spectroscopy. However, our analysis demonstrates that the photometric metallicity and the distance can be derived with comparable accuracy to those from the spectroscopic studies. In all cases, the fitting errors are smaller than the total systematic errors by factors of $2-3$ as shown in the tables.

The cluster richness can also affect the distance determination. If there are $N$ genuine single cluster members with random photometric errors in colors of $\epsilon$, the error in distance would be approximately $s \epsilon(3 N)^{-0.5} \approx 2 \epsilon N^{-0.5}$, where $s$ is a typical slope of MS. This error was already included in the fitting error, but its contribution is small for clusters with good photometry and $N \approx$ 100 . However, information on single cluster members is only available for a handful of open clusters, and the effects of binaries and foreground/background stars should be taken into account in distance estimation, as is discussed in the next two sections.

\subsection{Cluster Binaries}

The filtering algorithm ( $\S 4.1$ ) removes cluster binaries if they are sufficiently far from the MS. However, low mass ratio bi- naries would remain and might systematically reduce the estimated distance since they are brighter and redder than single cluster members.

To determine the size of the bias in distance estimation and to evaluate the effectiveness of the filtering algorithm, we performed artificial cluster CMD tests using a solar metallicity isochrone for an age of $550 \mathrm{Myr}$. We constructed each set of CMDs by generating 200 single stars or unresolved binaries in the color range used for the MS fitting, $0.4 \leq(B-V)_{0} \leq 1.3$. Primaries and secondaries in the binaries, as well as single MS stars, were randomly drawn from the mass function (MF) for M35 (Barrado y Navascués et al. 2001). The lower mass limit for the secondaries was set to be the hydrogen-burning limit at $0.08 M_{\odot}$. Each simulation is characterized by the binary fraction $(\mathrm{BF})$, which is defined as the number of binaries divided by the total number of systems in the above fitting range. For example, at $\mathrm{BF}=50 \%$, onehalf of the data points correspond to single stars and the other half represent unresolved binaries. We neglected multiple systems other than binaries due to the observed low frequency among solar-type stars (Duquennoy \& Mayor 1991). We assigned equal photometric errors of $0.01 \mathrm{mag}$ in $V, B-V, V-I_{\mathrm{C}}$, and $V-K_{S}$ and displaced them from the isochrone assuming a normal distribution. The artificial CMDs were then filtered, and the distance was derived in the usual way.

Figure 15 summarizes the results of these simulations. The top three panels show the bias in the distance modulus as a function of BF. The thick solid line shows the median of the bias from 200 artificial cluster CMDs computed at each BF with intervals of $5 \%$. Thin lines on either side are the first and third quartiles of these distributions. The dashed line indicates $\Delta(m-M)_{0}=0$. The vertical error bar displays the quartile ranges for a $\mathrm{BF}$ of $50 \%$, but with errors for each star of $0.03 \mathrm{mag}$.

The bottom panel of Figure 15 displays the ratio of the number of data points remaining after filtering compared to the input number of single stars in the simulation. The lines have the same meaning as in the other panels. For example, at $\mathrm{BF}=50 \%$, the simulation yields $N_{\text {fit }} / N_{\text {single }} \simeq 1.3$ after filtering, indicating that $\sim 70 \%$ of binaries were eliminated by the filtering algorithm.

As seen in Figure 15, the distance from the $(B-V, V) \mathrm{CMD}$ is less affected by the remaining binaries than those from $\left(V-I_{\mathrm{C}}, V\right)$ and $\left(V-K_{s}, V\right)$. Since stars were rejected if they 


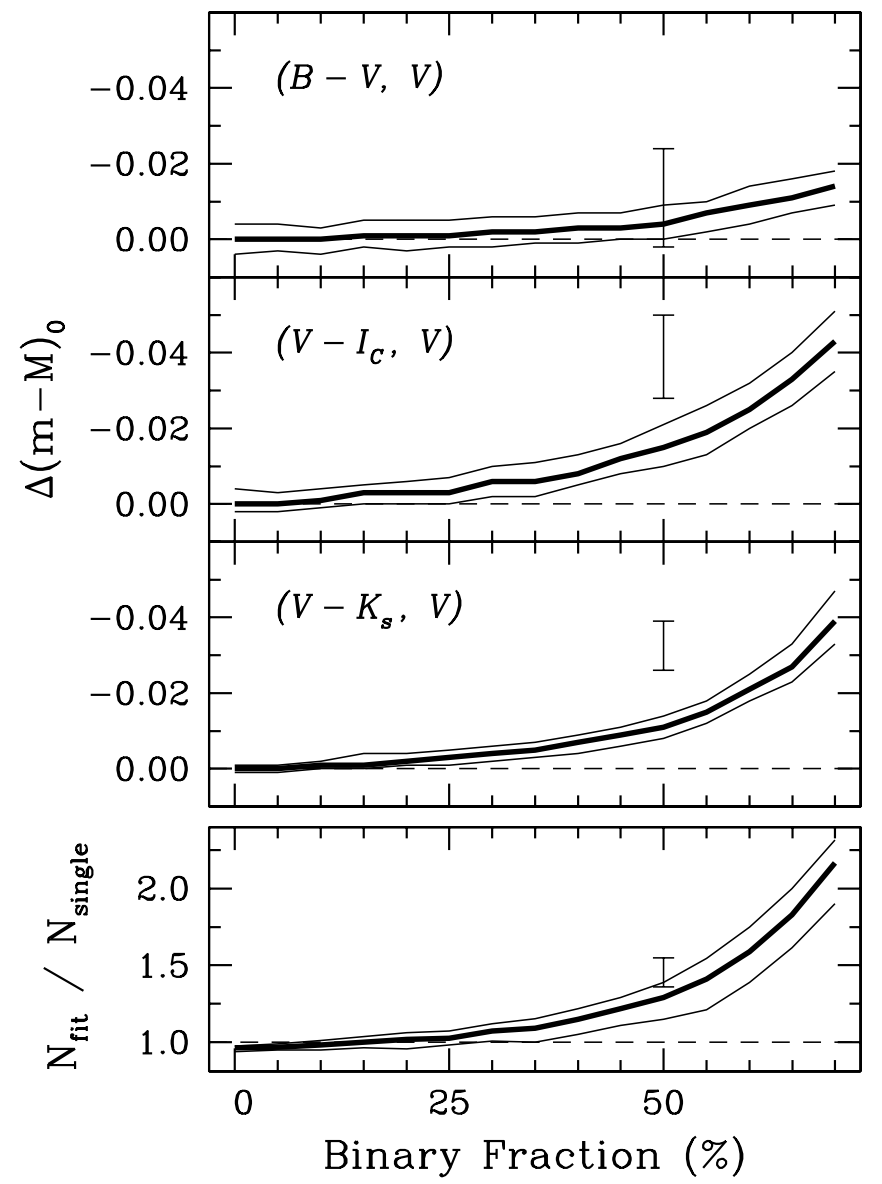

FIG. 15.-Effects of remaining binaries after photometric filtering from artificial cluster CMD tests (see text for details). Top three panels: Bias in distance modulus as a function of binary fraction in each CMD with assumed photometric errors of $0.01 \mathrm{mag}$. Bottom: Number ratio of all data points used in the fit to the input single stars. The thick solid line shows the median of these values from 200 artificial cluster CMDs at each binary fraction with intervals of 5\%, and thin lines on either side are the first and third quartiles. The dashed line indicates $\Delta(m-M)_{0}=0$. The differences are in the sense of shorter distances and more remaining binaries at a higher binary fraction. The vertical error bar displays the quartile ranges for a binary fraction of $50 \%$, but with errors for each star of 0.03 mag.

were filtered at least in one of the CMDs, the total number of remaining binaries is the same in the three CMDs with a binary detection limit set by a certain binary mass ratio ${ }^{13}(\sim 0.4$ in our simulation). However, binaries stand out more prominently in $\left(V-I_{\mathrm{C}}, V\right)$ or $\left(V-K_{s}, V\right)$ than in $(B-V, V)$ because the addition of the cooler secondaries results in a smaller change in the combined color in $B-V$ than in the other two color indices. As also seen in Figure 15, larger photometric errors result in a bigger bias in distance estimation since binaries are more difficult to identify when the photometric errors are larger.

The effects of binaries on the determination of the photometric metallicity and reddening are displayed in Figure 16. The top to bottom panels display the biases in $[\mathrm{M} / \mathrm{H}]_{E},[\mathrm{M} / \mathrm{H}]_{\chi^{2}}$, and $E(B-V)_{\chi^{2}}$, respectively. Error bars at $\mathrm{BF}=50 \%$ show the results with the photometric errors of $0.03 \mathrm{mag}$. As the BF increases, the MS fitting gives lower metallicity estimates. This is a direct result of the systematic errors in distance as seen in Figure 15. Recall that if an isochrone at a particular metallicity yields a distance from $(B-V, V)$ that is longer than from $\left(V-I_{\mathrm{C}}, V\right)$ or $\left(V-K_{s}, V\right)$, the photometric metallicity is smaller than that

\footnotetext{
13 Binary mass ratio is defined as the mass of the secondary (less massive) star divided by the mass of the primary star.
}

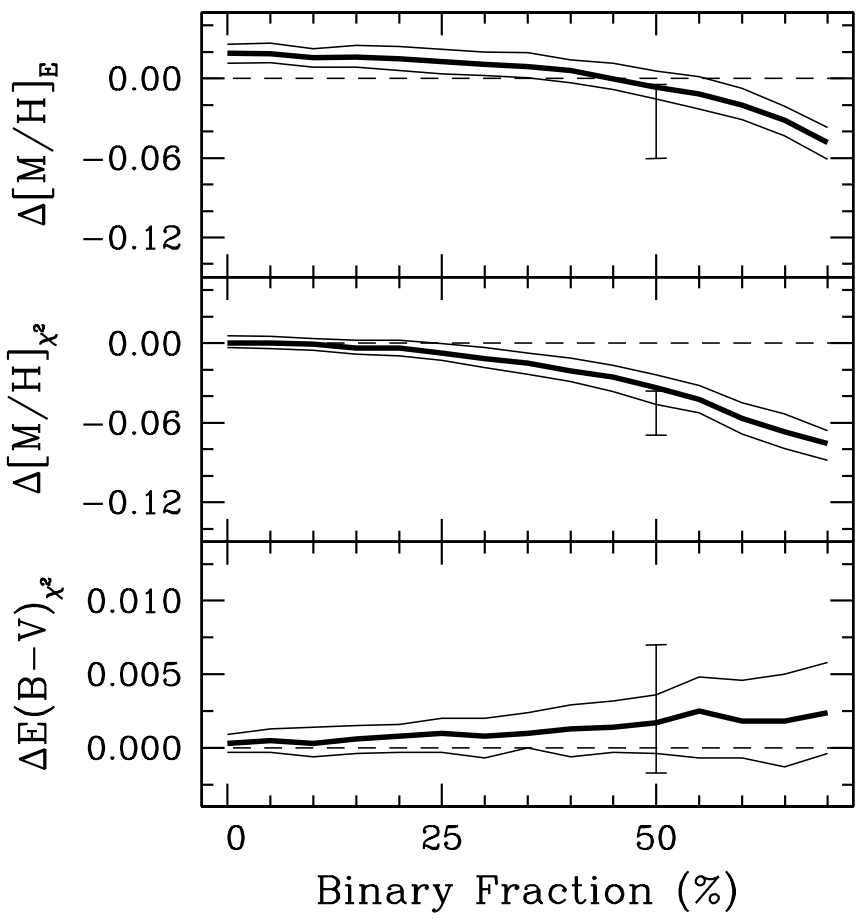

FIG. 16. - Same as Fig. 15, but for the bias in $[\mathrm{M} / \mathrm{H}]_{E},[\mathrm{M} / \mathrm{H}]_{\chi^{2}}$, and $E(B-V)_{\chi^{2}}$ (top to bottom). The differences are in the sense of a lower metallicity and a higher reddening estimate at a higher binary fraction.

of the isochrone. Increasing the BF leaves the distances from $(B-V, V)$ relatively unaltered but gives shorter distances in the other two colors, thus yielding the lower photometric metallicity. Nevertheless, the amount of a bias in the photometric metallicity is $\Delta[\mathrm{M} / \mathrm{H}]_{\chi^{2}} \approx-0.03$ at $\mathrm{BF}=50 \%$. In comparison, we conducted some experiments in which the filtering was not performed and found that this reduced the metallicity estimates by $\Delta[\mathrm{M} / \mathrm{H}]_{\chi^{2}} \approx-0.30$. The results from the bottom panel show that the reddening determination is almost unaffected by the presence of unfiltered binaries.

In Figure 16 a small offset of $\Delta[\mathrm{M} / \mathrm{H}]_{E} \approx 0.02$ at $\mathrm{BF}=0 \%$ shows the systematic error that was already included in the "calibration" error in $[\mathrm{M} / \mathrm{H}]_{E}$ (Tables 9-12). This happens because we assumed a linear relation between metallicity and distance over a wide range of metallicity (e.g., Fig. 5); higher order terms in the relation may be required to eliminate this systematic offset.

Table 13 summarizes the predicted bias in the distance, photometric metallicity, and reddening induced by unfiltered binaries at $\mathrm{BF}=50 \%$, which is typical of open clusters (Patience et al. 2002). We compare results by adopting different MFs for secondaries, as listed in the first column. We further tested several combinations of the various MFs including the Kroupa et al. (1993) MF for both primaries and secondaries. However, we found that the detailed form of the MF has no greater impact on the distance determination than the internal precision of photometry. The adopted random errors in the photometry are shown in the second column, while the remaining columns display the systematic errors.

In Praesepe, many solar-type stars have been observed for binarity from IR speckle, direct imaging, and spectroscopic studies, covering a wide range of orbital periods (Mermilliod \& Mayor 1999; Bouvier et al. 2001; Patience et al. 2002). As a result, its MS (Figs. 4 and 6) is fairly clean, suggesting that our binarity information in this cluster is almost complete at least for solar-type stars. In $0.4 \leq(B-V)_{0} \leq 0.8$, there are 46 known binaries out 
TABLE 13

Bias Induced by Unfiltered Binaries from Artificial Cluster Test $\mathrm{AT} \mathrm{BF}=50 \%$

\begin{tabular}{cccccc}
\hline \hline $\mathrm{MF}^{\mathrm{a}}$ & $\sigma_{\text {phot }}$ & $\Delta(m-M)_{0}$ & $\Delta[\mathrm{M} / \mathrm{H}]_{E}$ & $\Delta[\mathrm{M} / \mathrm{H}]_{\chi^{2}}$ & $\Delta E(B-V)_{\chi^{2}}$ \\
\hline $\mathrm{M} 35 \ldots \ldots \ldots \ldots .$. & \pm 0.01 & -0.010 & -0.007 & -0.033 & +0.002 \\
$\mathrm{M} 35 \ldots \ldots \ldots \ldots .$. & \pm 0.03 & -0.028 & -0.034 & -0.050 & +0.003 \\
Flat .............. & \pm 0.01 & -0.006 & +0.004 & -0.020 & +0.002 \\
Salpeter $\ldots . . . .$. & \pm 0.01 & -0.010 & -0.006 & -0.023 & +0.000 \\
\hline
\end{tabular}

a Stellar mass function for secondaries. Primaries were generated from M35 mass function (Barrado y Navascués et al. 2001).

b Salpeter (1955).

of 116 systems, yielding a BF of $40 \%$. At this value, our simulation results in Figures 15 and 16 yield $\Delta(m-M)_{0, S}=-0.006$, $\Delta[\mathrm{M} / \mathrm{H}]_{E}=-0.014, \Delta[\mathrm{M} / \mathrm{H}]_{\chi^{2}}=-0.037$, and $\Delta E(B-V)=$ +0.003 . These values are consistent with those from the comparison of two subsamples, computed before and after excluding known cluster binaries (Tables 7 and 8).

The Pleiades has also been extensively surveyed on binarity (e.g., Mermilliod et al. 1992; Bouvier et al. 1997). However, there are many stars remaining above the MS even after excluding all of the known binaries, suggesting that many binaries were probably left undetected by the previous surveys (see Fig. 7). In NGC 2516, there exists limited information on binarity only for B- and A-type stars from the spectroscopic studies (Abt \& Levy 1972; González \& Lapasset 2000).

To estimate the BF for each cluster, we first counted the total number of stars that were $0.3-1.0$ mag brighter in $V$ than the cluster MS in $(B-V, V)$. For NGC 2516, we subtracted field foreground/ background stars based on the distribution of $(m-M)_{0}$ (see next section) from the JTH01 catalog before counting likely cluster binaries. Within the above $\Delta V$ range, the observed BFs are $19 \% \pm 5 \%$ for the Pleiades and $18 \% \pm 2 \%$ for NGC 2516 , while that of Praesepe is $19 \% \pm 4 \%$. If we combine these estimates with the total observed BF of $40 \%$ for Praesepe, the total BFs for the Pleiades and NGC 2516 would be $38 \% \pm 11 \%$ and $38 \% \pm 4 \%$, respectively. The errors represent those from counting statistics.

Previous studies have also noted that Praesepe and the Pleiades have the similar BF among G- and K-type stars over a certain range of orbital periods (Mermilliod \& Mayor 1999; Bouvier et al. 2001; Patience et al. 2002). However, the above BF estimates based on the observed fraction of stars with $\Delta V \geq 0.3$ are uncertain for several reasons. For example, the distribution of binary mass ratio or the secondary MF can be different from one cluster to another. If we assume a flat MF for secondaries, we would derive $\mathrm{BF} \approx 50 \%$. On the other hand, the M35 MF predicts quite a high $\mathrm{BF}(>90 \%)$ and produces too many binaries near MS (low mass ratio binaries) compared to the observed distribution of stars. It should also be noted that the BF in the simulation only concerns photometric binaries. In other words, blending of physically unrelated stars could increase the $\mathrm{BF}$, while binaries with large angular separations could reduce the BF. As a result, the BF depends on a number of cluster properties, as well as a specific design of a photometric survey. In addition, a substantial fraction of binaries tend to have equal-mass companions (e.g., Halbwachs et al. 2003; Pinsonneault \& Stanek 2006 and references therein). These binaries would be well separated from the cluster MS, so they can be easily detected and removed by the filtering algorithm. The net effect would be an overestimation of the bias in distances and photometric metallicities in the simulation for a given BF.

From the above considerations, we adopted $30 \%-50 \%$ as the range of $\pm 1 \sigma$ formal errors in the BF for the Pleiades and NGC

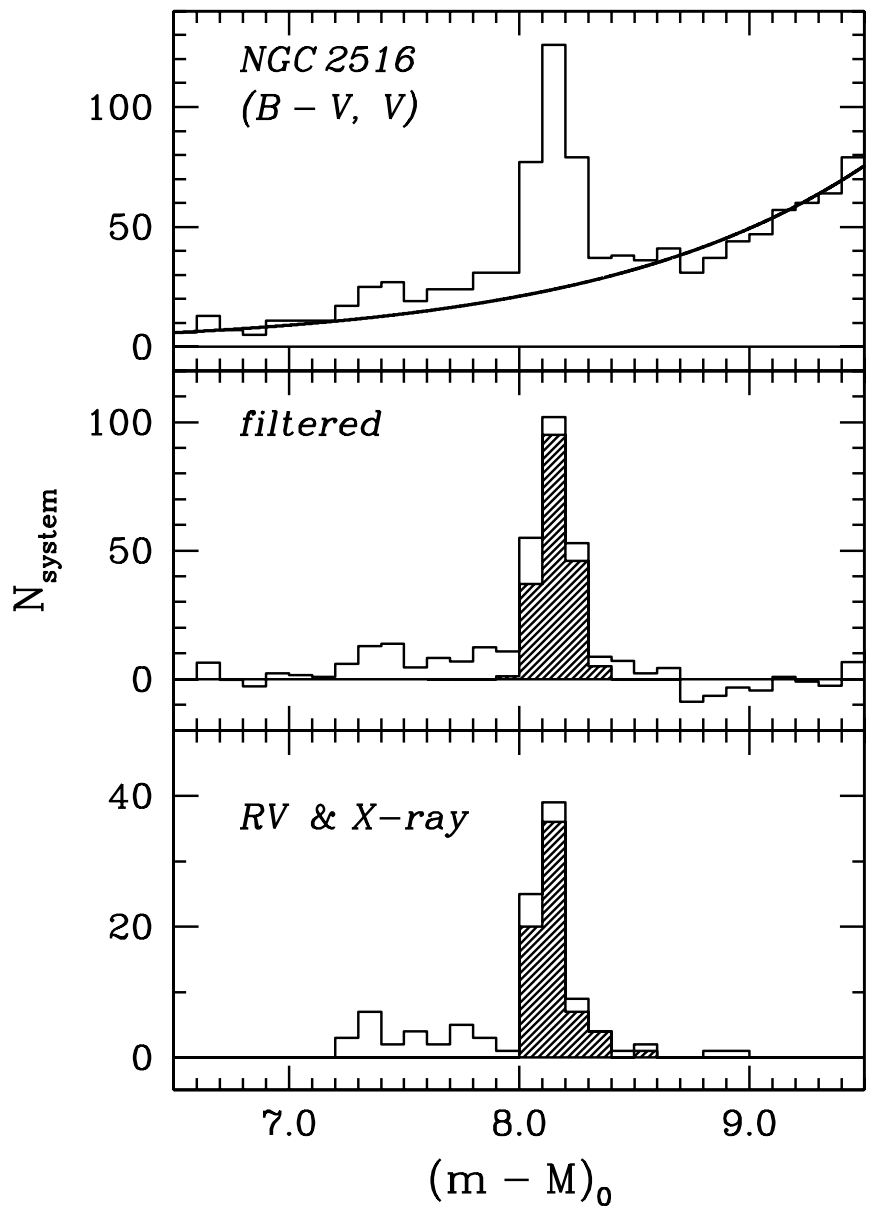

FIG. 17.-Distribution of individual distance moduli for NGC 2516 from the JTH01 photometry in $(B-V, V)$. Plotted are the number of stars per $0.1 \mathrm{mag}$ interval in distance modulus. The same isochrone and color fitting ranges in Fig. 9 were used. Top: Distribution of the full data in the catalog. The solid line is an exponential fit to the histogram well beyond the MS and the binary sequence to model the field star distribution. Middle: The hatched histogram represents the distribution of the stars that remained after filtering. For comparison, the distribution of the full data set after subtracting backgrounds with the exponential curve in the top panel is shown as an open histogram. Bottom: The open histogram is a summed distribution of the radial velocity members (Terndrup et al. 2002) and the Chandra X-ray-detected sources (Damiani et al. 2003), and the distribution of these samples after filtering is shown as a hatched histogram.

2516. From the simulation result ( $0.01 \mathrm{mag}$ error, M35 MFs for both binary components), this BF yields $\Delta(m-M)_{S}=-0.007 \pm$ $0.003, \Delta[\mathrm{M} / \mathrm{H}]_{E}=-0.020 \pm 0.015, \Delta[\mathrm{M} / \mathrm{H}]_{\chi^{2}}=-0.036 \pm$ 0.013 , and $\Delta E(B-V)_{\chi^{2}}=+0.003 \pm 0.001$. These values should be subtracted from the MS fitting results in Tables 7 and 8 to correct the effects of binaries. Additional simulations showed that $\Delta[\mathrm{M} / \mathrm{H}]_{\chi^{2}}=+0.008 \pm 0.004$ and $\Delta E(B-V)_{\chi^{2}}=+0.003 \pm$ 0.001 in the case of the fixed cluster distance. Bias in distance at the photometric metallicity would be the quadrature sum of $\Delta(m-M)_{S}$ and the error contributions from $\Delta[\mathrm{M} / \mathrm{H}]_{\chi^{2}}$ (or $\left.\Delta[\mathrm{M} / \mathrm{H}]_{E}\right)$ and $\Delta E(B-V)_{\chi}$.

\subsection{Field Star Contamination}

In clusters such as NGC 2516 (Fig. 9), a significant number of foreground/background stars are present near the MS. Because the number density of these stars in each CMD increases toward fainter magnitudes, the distance from MS fitting can be systematically overestimated.

In Figure 17 we show the distribution of individual distance moduli for NGC 2516 from the JTH01 photometry in $(B-V, V)$. 
TABLE 14

Summary of Open Cluster Parameters

\begin{tabular}{|c|c|c|c|c|}
\hline Parameter & Praesepe & Pleiades & M67 & NGC 2516 \\
\hline \multicolumn{5}{|c|}{$[\mathrm{Fe} / \mathrm{H}]$} \\
\hline This paper ${ }^{\mathrm{a}} \ldots$. & $+0.20 \pm 0.04$ & $+0.04 \pm 0.05^{\mathrm{b}}$ & $-0.02 \pm 0.05^{\mathrm{c}}$ & $-0.07 \pm 0.06^{\mathrm{d}}$ \\
\hline 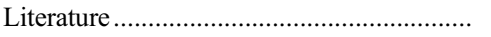 & $+0.11 \pm 0.03$ & $+0.04 \pm 0.02$ & $+0.00 \pm 0.01$ & $+0.01 \pm 0.07$ \\
\hline Weighted mean ........................................... & $+0.14 \pm 0.02$ & $+0.04 \pm 0.02$ & $+0.00 \pm 0.01$ & $-0.04 \pm 0.05$ \\
\hline \multicolumn{5}{|c|}{$E(B-V)$} \\
\hline 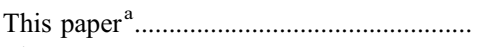 & $-0.003 \pm 0.008$ & $0.022 \pm 0.014^{\mathrm{b}}$ & $0.042 \pm 0.019^{\mathrm{c}}$ & $0.125 \pm 0.014^{\mathrm{d}}$ \\
\hline 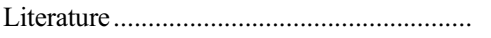 & $0.007 \pm 0.002$ & $0.032 \pm 0.003$ & $0.041 \pm 0.004$ & $0.117 \pm 0.002$ \\
\hline Weighted mean .......................................... & $0.006 \pm 0.002$ & $0.032 \pm 0.003$ & $0.041 \pm 0.004$ & $0.117 \pm 0.002$ \\
\hline \multicolumn{5}{|c|}{$(m-M)_{0}$} \\
\hline 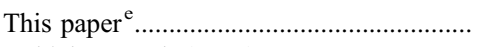 & $6.33 \pm 0.04$ & $5.63 \pm 0.02^{\mathrm{f}}$ & $9.61 \pm 0.03^{c}$ & $8.03 \pm 0.04^{\mathrm{d}}$ \\
\hline Robichon et al. (1999)............................... & $6.28 \pm 0.13$ & $5.36 \pm 0.06$ & $\ldots$ & $7.70 \pm 0.16$ \\
\hline 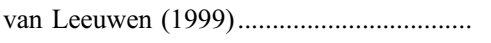 & $6.37 \pm 0.15$ & $5.37 \pm 0.07$ & $\cdots$ & $\ldots$ \\
\hline \multicolumn{5}{|c|}{$\begin{array}{l}\text { a Solution from the } \chi^{2} \text { minimization. } \\
\text { b Solution at the average geometric distance (Table 1). } \\
\text { c Average solution from MMJ93 and } \mathrm{S} 04 \text { photometry with single cluster membership by S04. } \\
\text { d Average solution from JTH01 and } \mathrm{S} 02 \text { photometry. } \\
\text { e Estimated at the weighted mean }[\mathrm{Fe} / \mathrm{H}] \text { and } E(B-V) \text {. } \\
\text { f Average geometric distance from Table } 1 \text {. }\end{array}$} \\
\hline
\end{tabular}

Plotted are the number of stars per 0.1 mag interval in distance modulus. The same isochrone and color fitting ranges in Figure 9 were used. In the top panel, the histogram represents the number of stars in the catalog before photometric filtering. It shows the density peak that represents the cluster MS, the binary sequence that extends toward shorter distance from the MS, and the field star distribution that was fitted by an exponential function (solid line). The fit excluded stars near the MS and the cluster binaries and was performed over a wider range in $(m-M)_{0}$ than shown here. In the middle panel, the hatched histogram represents the distribution of the stars that remained after filtering from the catalog. This is compared with an open histogram, which was found by subtracting the exponential curve from the histogram of the full data in the top panel. In the bottom panel we show the summed distribution of the RV members (Terndrup et al. 2002) and the Chandra X-ray-detected sources (Damiani et al. 2003) as an open histogram. The distribution of these stars after filtering is shown as a hatched histogram.

One of the most conspicuous features in the middle panel of Figure 17 is the effective removal of cluster binaries from filtering. This can be seen from a deficit of data points in the hatched histogram against the open one at shorter distances from the MS. In addition, good matches are found between these two histograms near the MS, indicating that the filtering algorithm has worked correctly in the foreground/background subtraction.

The RV and X-ray samples contain many cluster binaries (Fig. 17, bottom panel), but they are mostly free from field star contamination. On the other hand, the full catalog data (Fig. 17, top panel) are contaminated by both cluster binaries and foreground/ background stars. Therefore, the effects of field star contamination after filtering can be estimated by comparing distances, metallicities, and reddening values derived from each filtered set of the data (Fig. 17, hatched histograms). From Tables 7 and 8, the weighted mean differences in these quantities from the JTH01 and S02 photometry are $\Delta(m-M)_{S}=+0.010 \pm 0.002, \Delta[\mathrm{M} / \mathrm{H}]_{E}=$ $+0.020 \pm 0.003, \Delta[\mathrm{M} / \mathrm{H}]_{\chi^{2}}=+0.038 \pm 0.031$, and $\Delta E(B-$ $V)_{\chi^{2}}=-0.003 \pm 0.007$. The errors are half of the differences in the estimated bias from the two photometry. Within the estimated errors, these values are of the same size but opposite di- rection as the binary corrections at $\mathrm{BF}=40 \%$. In other words, these two biases cancel out each other.

It is possible that the RV or X-ray samples are more heavily contaminated by cluster binaries than the full catalog data. However, we found that they have a similar fraction of stars $(\sim 19 \%)$ in $0.3 \mathrm{mag} \leq \Delta V \leq 1.0 \mathrm{mag}$ above the MS. This implies that the RV and $\mathrm{X}$-ray samples would have a similar total BF unless they have an excess of low mass ratio binaries.

\subsection{Comparison with Previous Estimates}

In Table 14 we summarize our metallicity and reddening estimates from the $\chi^{2}$ minimization and compare them with the literature values. The $[\mathrm{M} / \mathrm{H}]$ and $E(B-V)$ for the Pleiades are those derived at the average geometric distance in Table 1 . These estimates for the Pleiades were also corrected for the bias induced by the binary contamination. This correction was applied to the result from the data set that originally contained all known binaries. For NGC 2516, we did not apply the bias correction for both binaries and field star contamination for the reason explained in the previous section. The errors are the quadrature sum of the fitting and total systematic errors in Tables 9-12. For the Pleiades and NGC 2516, we additionally include the errors in the bias from binaries. Our estimates for M67 and NGC 2516 are the weighted averages from the two photometric data sets for each cluster. We adopted half of the difference in these estimates as the $1 \sigma$ error in the case where they do not agree with each other within the estimated errors. In the third row of each quantity, weighted averages of our estimates and those in the literature are shown; however, the averages remain close to the literature values in most cases. Our estimated distances at these average metallicities and reddening values are shown and compared with the Hipparcos measurements (Robichon et al. 1999; van Leeuwen 1999) in the last three rows. We discuss the Hipparcos measurements in $\S 7.2$.

In the top panel of Figure 18 we compare the photometric metallicities with the spectroscopic values in Table 14. The comparison yields $\left\langle[\mathrm{M} / \mathrm{H}]_{\chi^{2}}-[\mathrm{Fe} / \mathrm{H}]_{\text {spec }}\right\rangle=+0.01 \pm 0.03$ (s.e.m.). Although our photometric metallicity for Praesepe is $\sim 2 \sigma$ higher than the spectroscopic value, the $\chi^{2}$ with respect to the identity 

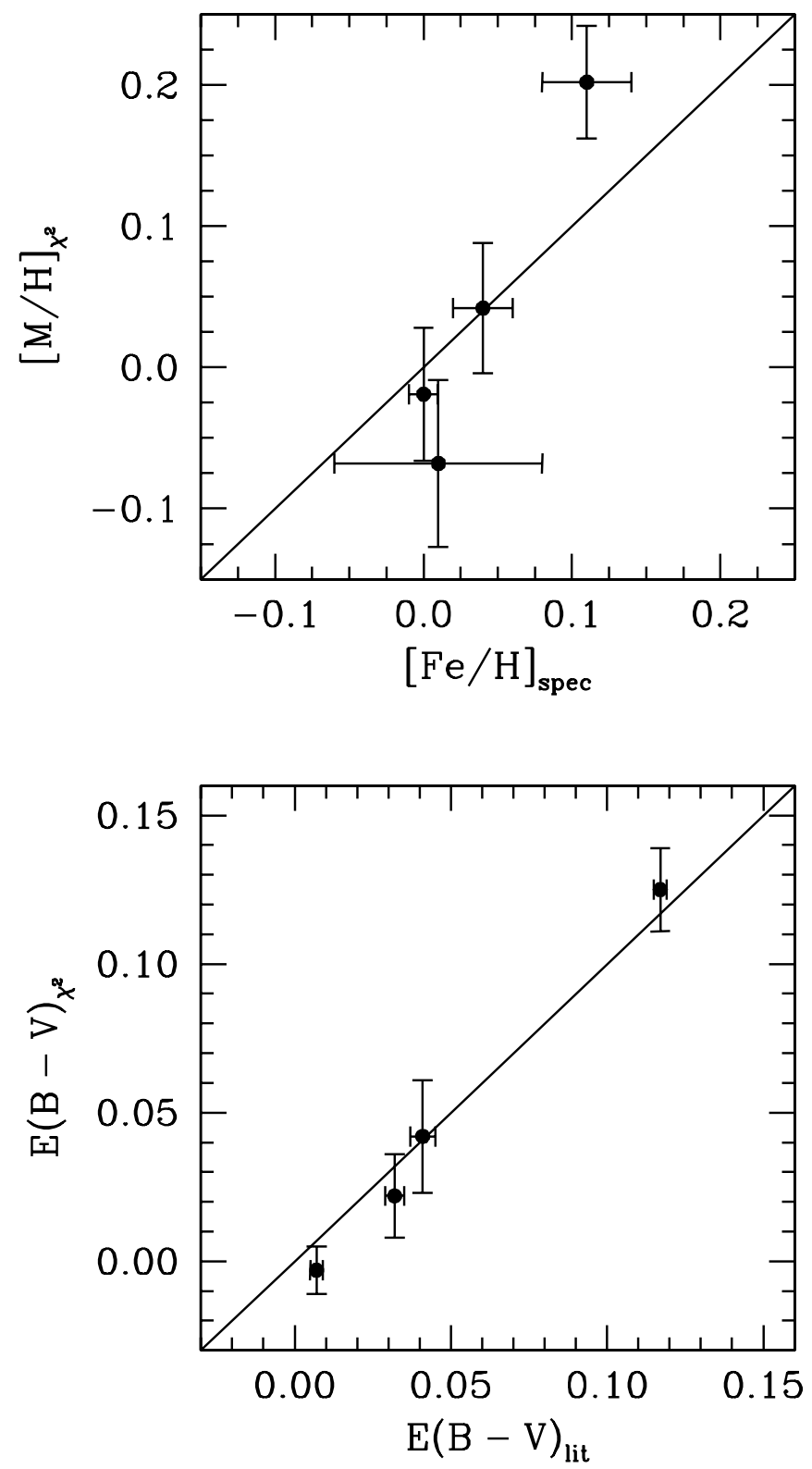

FIG. 18.-Comparison of the photometric $[\mathrm{M} / \mathrm{H}]_{\chi^{2}}$ and the spectroscopic $[\mathrm{Fe} / \mathrm{H}](t o p)$ and that of the photometrically determined $E(B-V)_{\chi^{2}}$ and the literature value (bottom) from Table 14. The diagonal lines indicate identity.

line is 4.3 from these four cluster points. In other words, the errors in $[\mathrm{M} / \mathrm{H}]_{\chi^{2}}$ were correctly estimated. We investigate a photometric metallicity scale over a wider range of $[\mathrm{Fe} / \mathrm{H}]$ in the next paper of this series (D. M. Terndrup et al. 2007, in preparation).

The $E(B-V)$ estimates from the $\chi^{2}$ minimization are plotted against the literature values in the bottom panel of Figure 18. Here the mean difference between the two measures is $\langle E(B-$ $\left.V)_{\chi^{2}}-E(B-V)_{\text {lit }}\right\rangle=-0.006 \pm 0.012$ (s.e.m.). The $\chi^{2}$ is 2.4 , which again indicates that the errors were estimated correctly. However, our photometric reddening from $B V I_{\mathrm{C}} K_{s}$ data is not completely independent from the literature values from $U B V$ data since most of the literature estimates in Table 6 are from the similar methodology that uses the observed colors of the unreddened MS stars. We did not include $U$-band measurement in the isochrone calibration because of its low reproducibility and relatively large systematic errors among different studies (Bessell

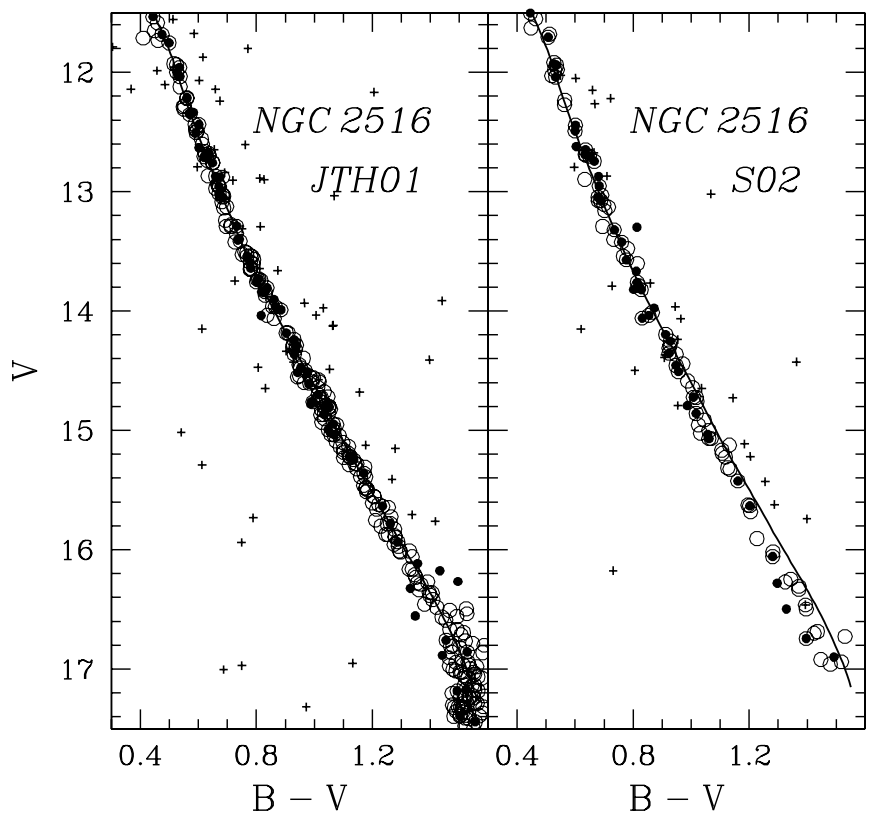

FIG. 19.- Two different interpretations on young K dwarfs in NGC 2516. Photometry of JTH01 (left) and that of S02 (right) are shown. Open circles are the stars that remained after photometric filtering from the full catalogs. Filled circles and plus signs show the stars that are RV members (Terndrup et al. 2002) or X-ray-detected sources (Damiani et al. 2003) if they were accepted or rejected, respectively, by the filtering on these samples. The same isochrone and color fitting ranges in Fig. 9 were used. Note that stars with the S02 photometry are bluer than the isochrone for $B-V \gtrsim 1.0$.

2005 and references therein). These properties make it difficult to reliably use the $U$-band data in the high-precision distance estimates as in this paper.

\section{OTHER ISSUES}

\subsection{Are the K Dwarfs in NGC 2516 Blue, Too?}

In Figure 19 we display the MS of NGC 2516 in $(B-V, V)$, which at an age of 140 Myr may exhibit the phenomenon of blue K dwarfs as seen in the Pleiades CMD (Stauffer et al. 2003; see also Fig. 7). The left panel displays the photometry of JTH01 in $(B-V, V)$ while the right panel shows that of $\mathrm{S} 02$. In each panel, the open circles are those stars that remained after photometric filtering. The filled circles and plus signs show the stars that are RV members (Terndrup et al. 2002) or X-ray-detected sources (Damiani et al. 2003) if they were accepted or rejected, respectively, by the filtering.

The solid lines in Figure 19 are $140 \mathrm{Myr}$ isochrones with $[\mathrm{Fe} / \mathrm{H}]=+0.01$ and $E(B-V)=0.117$, fitted over $0.40 \leq$ $(B-V)_{0} \leq 0.80$. The isochrone matches the JTH01 photometry down to at least $V \approx 17$, but the S02 photometry is bluer than the isochrone for $B-V \gtrsim 1.0\left(M_{V} \gtrsim 6.5\right)$. This systematic difference was previously noted when we compared the photometry from the two studies (Fig. 2).

The individual distance modulus for each star is shown in Figure 20, plotted against $(B-V)_{0}$, along with the Pleiades and Praesepe stars that have similar colors. The solid line in each panel is the median value from fits over $0.4 \leq(B-V)_{0} \leq 0.8$ (note that our analysis above used the full color range for Praesepe). While the JTH01 data show a flat distribution over $0.4 \leq(B-$ $V)_{0} \leq 1.3$ as in Praesepe, the red stars in the S02 data systematically trend toward longer distances for $(B-V)_{0} \gtrsim 0.8$ where the Pleiades $\mathrm{K}$ dwarfs also depart from the isochrone. 


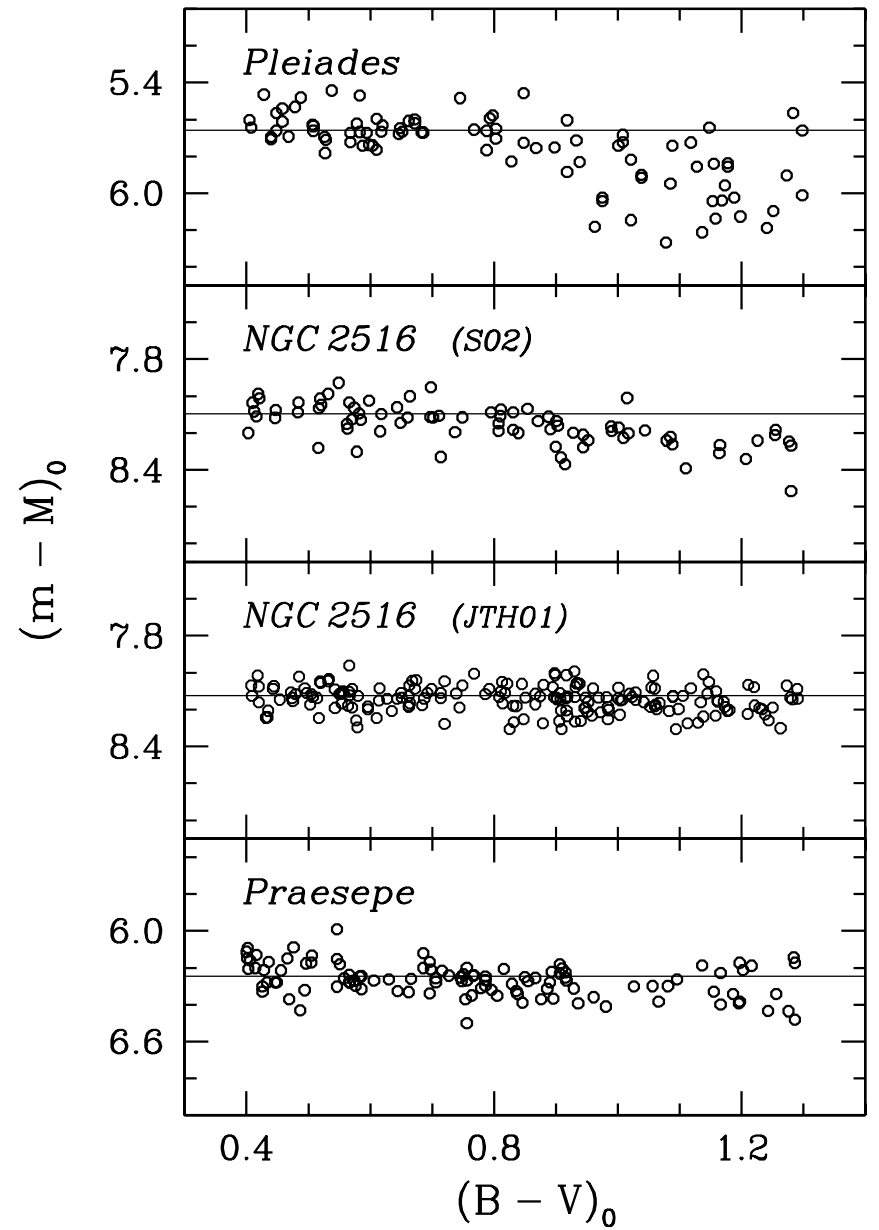

FIG. 20.- Individual distance moduli of stars in the Pleiades (top), NGC 2516 (middle), and Praesepe (bottom) from $(B-V, V)$ with respect to the isochrones with spectroscopic metallicities and reddening values in the literature (Tables 5 and 6). Only stars that remained after filtering are shown. Horizontal lines indicate median distance moduli derived in $0.4 \leq(B-V)_{0} \leq 0.8$. While the JTH01 data show a flat distribution, the $\mathrm{S} 02$ data exhibit longer distances at redder colors where the Pleides $\mathrm{K}$ dwarfs also depart from the isochrone.

From these plots alone it is not possible to know whether the blue $\mathrm{K}$ dwarf phenomenon exists in NGC 2516 since the interpretation depends on which data set is used. Stauffer et al. (2003) argued that the departure from the ordinary MS in the Pleiades is connected with stellar rotation rate since the stars with the fastest rotation show the most anomalous colors (see their Fig. 15). In Figure 21 we display the difference in $(m-M)_{0}$ for stars in NGC 2516 from the average distance modulus in each CMD against the projected rotation velocity. The filled circles are from the JTH01 photometry, while the open circles are from S02. Positive $\Delta(m-M)_{0}$ indicates that stars are fainter (or bluer) than the isochrone, and the dashed line shows $\Delta(m-M)_{0}=0$. The $v \sin i$ data are only available up to $V \approx 15$ or $(B-V)_{0} \lesssim 1.0$ (Terndrup et al. 2002), and only stars with $(B-V)_{0} \geq 0.8$ are shown. Within this restricted color range, a few stars with rapid rotation in $(B-V, V)$ are about $0.1-0.2$ mag fainter than the slow rotators. By comparison, rapidly rotating stars with similar colors in the Pleiades are about 0.5 mag below the MS. This is suggestive that the stars in NGC 2516 may also exhibit the blue K dwarf phenomenon although the degree of departure is smaller than in the Pleiades. The opposite correlation is seen in $\left(V-K_{s}, V\right)$ as also noted by Stauffer et al. (2003), and we see no significant correlation in $\left(V-I_{\mathrm{C}}, V\right)$.

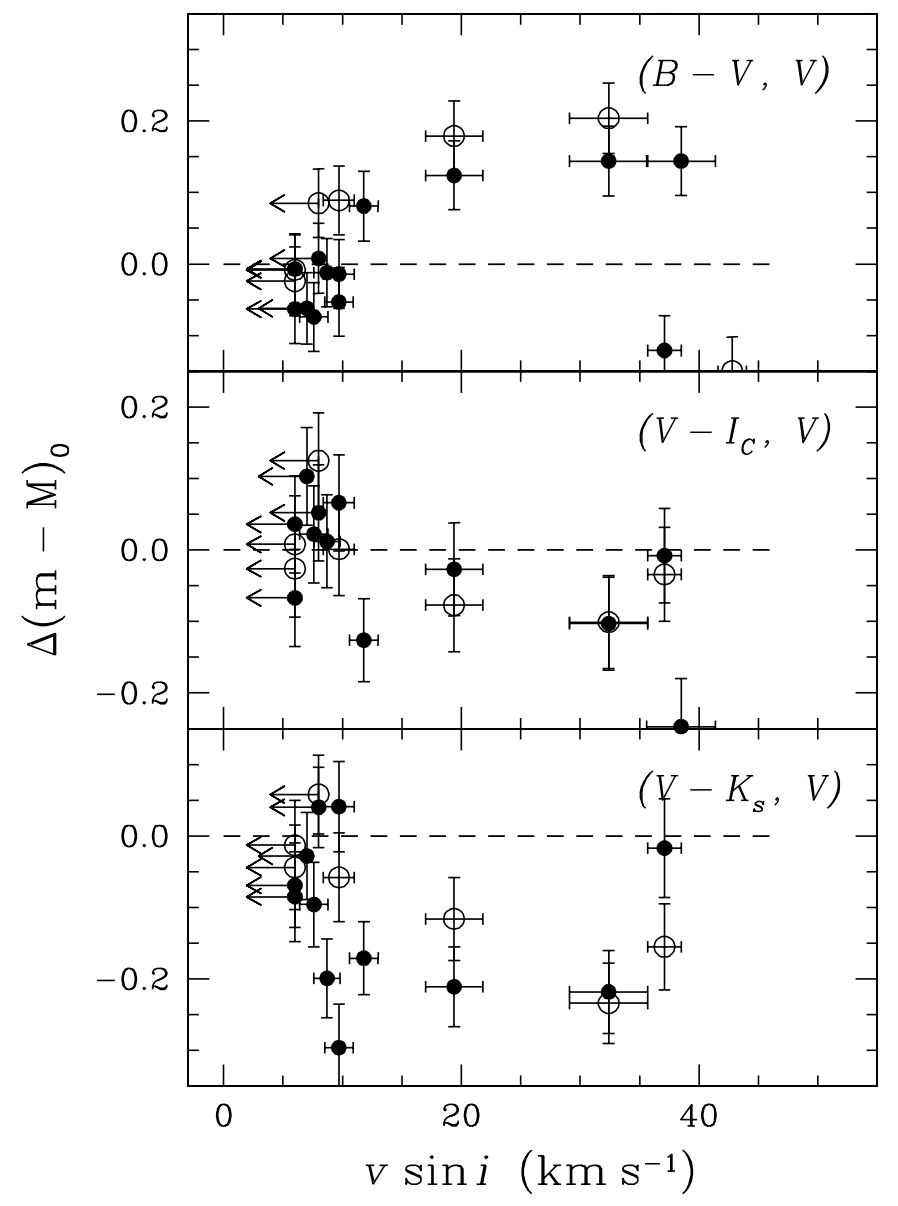

FIG. 21.-Dependence of the photometric anomalies of $\mathrm{K}$ dwarfs in NGC 2516 on the projected rotational velocity. The filled circles are from the JTH01 photometry, and the open circles are from the $\mathrm{S} 02$ for $0.8 \leq(B-V)_{0} \leq 1.0$. Displayed are the individual distance moduli with respect to the average distance in each CMD, and the dashed line shows $\Delta(m-M)_{0}=0$. The same isochrone and color fitting ranges in Fig. 9 were used. Positive $\Delta(m-M)_{0}$ indicates that stars are fainter than the isochrone.

\subsection{Hipparcos Distances}

In Figure 22 we compare our derived distance moduli with the Hipparcos parallax results from Robichon et al. (1999) for the Pleiades, Praesepe, and NGC 2516 (M67 was too distant for Hipparcos to measure its parallax). In the top panel, the open circles show the comparison with $(m-M)_{0, S}$ at the spectroscopic metallicity. The errors are the quadrature sum of the fitting and total systematic errors in Tables 9-12. For the Pleiades and NGC 2516, we additionally included the error in the bias correction for binaries. We also plot as an open triangle the comparison of $(m-M)_{0, S}$ and the geometric distance to the Pleiades from Table 1 and connect the two Pleiades points with a dashed line. The bottom panel shows the difference between the two distance measurements. The filled circles represent the comparison with $(m-M)_{0, \chi^{2}}$ and are shown without error bars.

While our distance to Praesepe shows a good agreement with the Hipparcos value, the 1999 Hipparcos distance to the Pleiades exhibits more than a $\sim 3 \sigma$ difference at either the spectroscopic or photometric metallicity even if we take into account all systematic errors. Similarly, NGC 2516 shows a $\sim 2 \sigma$ difference. These results extend the previous discussion by Pinsonneault et al. (1998) and Terndrup et al. (2002), but the current results are based on isochrones with improved color calibrations over wider color ranges and with additional $\left(V-K_{s}\right)$ colors. 


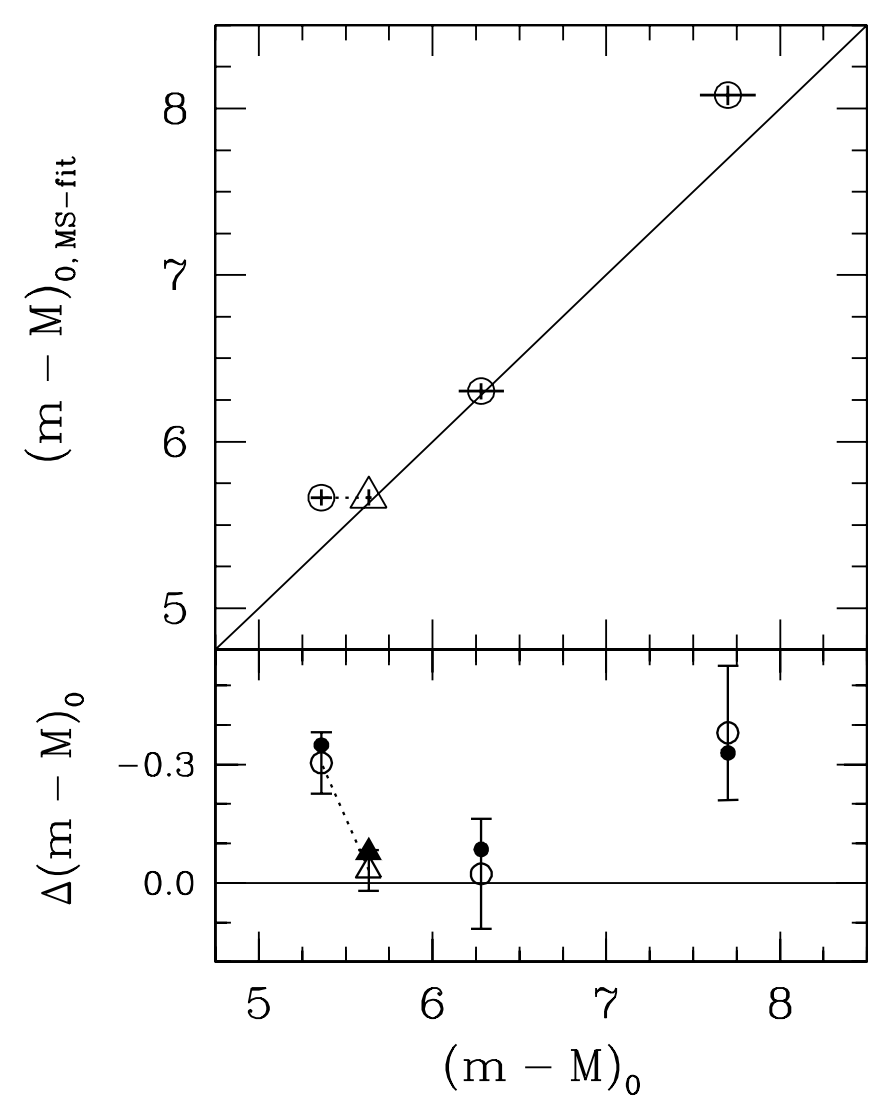

Fig. 22.-Comparison of the Hipparcos parallax results (Robichon et al. 1999) and our derived distance moduli. Open circles are those for the Pleiades, Praesepe, and NGC 2516 (left to right) when distances are determined at the spectroscopic metallicities. The open triangles are the comparison with the geometric distance to the Pleiades (Table 1), and the two Pleiades points are connected with a dashed line. The errors are the quadrature sum of the fitting and total systematic errors in Tables 9-12 and those in the bias induced by binaries. Filled symbols are the comparison with $(m-M)_{0, \chi^{2}}$ and are shown without error bars. The diagonal line indicates identity in the top panel.

In fact, Pinsonneault et al. (2000) derived a consistent distance to NGC 2516 with the Hipparcos value, but with a considerably lower photometric metallicity $([\mathrm{M} / \mathrm{H}]=-0.26)$ than presented in this paper. This happened because $B-V$ and $V-I_{\mathrm{C}}$ colors of their isochrones from the Yale color calibration (Green 1988) were affected by large systematic errors in the color- $T_{\text {eff }}$ relations. The photometric metallicity was redetermined to be $[\mathrm{M} / \mathrm{H}]=$ -0.05 by Terndrup et al. (2002), who employed the same colors, but with a preliminary calibration of the isochrones that later appeared in our Paper II. As in this paper, they found a distance to NGC 2516 that was longer than the Hipparcos value.

In the Pleiades, we achieved an excellent fit in all CMDs and derived a photometric metallicity and distance that are consistent with the spectroscopic determinations and the geometric distances from a variety of methods. In our analysis, we excluded $B-V$ colors where the cluster K dwarfs are anomalously blue. Percival et al. (2003) presented $B V(R I)_{\mathrm{C}}$ photometry for a sample of local stars with accurate Hipparcos parallaxes and developed a model-independent MS fitting method based on the relation between luminosity and metallicity for these stars. In order to select unevolved stars, however, they picked targets with $0.7 \lesssim$ $(B-V) \lesssim 1.0$ and found a Pleiades distance in $(B-V, V)$ that was longer than that from $\left(V-I_{\mathrm{C}}, V\right)$, indicating a photometric metallicity of $[\mathrm{M} / \mathrm{H}] \approx-0.3$. This led them to suggest that the spectroscopic metallicity of the Pleiades was in error, even though it is well established (Table 5). As they subsequently realized
(Percival et al. 2005), their analysis was seriously affected by the anomalous $B-V$ colors of the $\mathrm{K}$ dwarfs. In the later study, they showed that distances derived with $V-I_{\mathrm{C}}$ and $V-K_{s}$ were consistent with the correct distance for the Pleiades.

The disagreement with the Hipparcos values for the Pleiades and NGC 2516 has been attributed to a local zero-point error of the individual stellar parallaxes in the Hipparcos catalog (Pinsonneault et al. 1998; van Leeuwen 2005). A more elaborate reduction of the Hipparcos data (van Leeuwen \& Fantino 2005) would help to resolve the difference from the MS fitting distances.

\subsection{The Helium Abundance of the Pleiades}

Because the luminosity of the model isochrones at fixed $T_{\text {eff }}$ is sensitive to the helium abundance $\left(\partial M_{\mathrm{bol}} / \partial Y \approx 3\right)$, we can derive the helium abundance if the cluster distance and metallicity are known. The isochrones employed here assume that the Sun's metal abundance is $Z_{\odot}=0.0176$ and that the solar helium abundance in models lacking diffusion is $Y_{\odot}=0.266$. This yields $\Delta Y / \Delta Z=1.2$ with the primordial helium abundance of $Y_{p}=$ $0.245 \pm 0.002$ (see Paper I) ${ }^{14}$ We chose the solar models with no diffusion because microscopic diffusion would not be important for young clusters such as the Hyades and the Pleiades. In Paper I we derived the Hyades helium abundance, $Y_{\text {Hyades }}=$ $0.271 \pm 0.006$, using the Hipparcos distance to the Hyades (de Bruijne et al. 2001), combined with the metallicity estimate from Paulson et al. (2003) and the masses of the components of the eclipsing binary vB 22 (Torres \& Ribas 2002). Since the standard solar model including the effects of helium diffusion and rotational mixing implies an initial solar helium abundance of $Y_{\odot \text {,init }}=0.274$ (Bahcall et al. 2001), the Hyades abundance is equal within the errors to the Sun's value at the time of formation, even though the Hyades has $~ 35 \%$ higher metal content than the Sun.

At the spectroscopic metallicity for the Pleiades, $[\mathrm{Fe} / \mathrm{H}]=$ $+0.04 \pm 0.03$, we derived $(m-M)_{0, S}=5.664 \pm 0.041$, where the error is the quadrature sum of the fitting and total systematic errors from Table 10, excluding the error contribution from helium. It also includes the error in the bias correction for binaries. Our distance is longer than the geometric distance, $(m-M)_{0}=$ $5.632 \pm 0.017$ (Table 1), which indicates that the helium abundance of the Pleiades is higher than the assumed value in the model. Since the isochrones have $Y=0.268$ at the metallicity of the Pleiades, the difference between the two distances yields $Y=0.279 \pm 0.015$ for the Pleiades.

The Pleiades helium abundance is the same as both the initial solar and the Hyades' abundances within the errors. To know whether there is a dispersion in the local helium abundance, it would be necessary to reduce the size of the systematic errors in the MS fitting either by improving the photometric calibration or by obtaining more accurate cluster metal abundances. However, we can certainly rule out extreme helium abundances $(Y \approx 0.34)$ that had been advocated to explain the shorter Hipparcos distance to the Pleiades (Belikov et al. 1998). From the primordial helium abundance, the initial solar value, and the helium abundances for the Hyades and the Pleiades, we derive $\Delta Y / \Delta Z=1.42 \pm 0.17$.

When future astrometric missions such as Gaia (Perryman et al. 2001) provide highly accurate distances, the error in the helium abundance will be predominantly determined by the accuracy of the MS fitting. In such cases, cosmic scatter of $\Delta Y=0.02$ could be detectable with the current accuracy of the MS fitting technique. Furthermore, the error in the helium enrichment parameter

\footnotetext{
${ }^{14}$ We assumed that the helium abundance is a function of the heavyelement content $Z$ as given by $Y=Y_{p}+(\Delta Y / \Delta Z) Z$.
} 
scales as $\epsilon(\Delta Y / \Delta Z) \approx 0.3(N / 100)^{-0.5}\left(\sigma_{\mu} / 0.05 \mathrm{mag}\right)$, where $N$ is the total number of clusters and $\sigma_{\mu}$ is the error in the distance from the MS fitting. A more accurate value of $\Delta Y / \Delta Z$ requires that many input systematic errors in the MS fitting be kept under control. Stellar abundances in particular are susceptible to systematic errors from the adopted $T_{\text {eff }}$ scales. This issue will be addressed in the next paper of this series (D. M. Terndrup et al. 2007 , in preparation).

\section{SUMMARY AND DISCUSSION}

We have generated a set of isochrones near solar metallicity $(-0.3 \leq[\mathrm{Fe} / \mathrm{H}] \leq+0.2$ in 0.1 dex increments $)$ and have calibrated them by correcting the Lejeune et al. $(1997,1998)$ color- $T_{\text {eff }}$ relations to give a precise match to the Hyades MS at $[\mathrm{Fe} / \mathrm{H}]=$ $+0.13 \pm 0.01$ and $(m-M)_{0}=3.33 \pm 0.01$ (Paper II). We tested the hypothesis that the empirical color- $T_{\text {eff }}$ corrections improve the accuracy of distance estimation using the four wellstudied open clusters: Praesepe, the Pleiades, M67, and NGC 2516.

Either by adopting cluster metallicities and reddening values in the literature or by constraining them from the multicolor CMDs, we showed that the calibrated set of isochrones not only matches the cluster MS precisely but also gives internally consistent distances from $(B-V, V),\left(V-I_{\mathrm{C}}, V\right)$, and $\left(V-K_{s}, V\right)$ CMDs. The internal precision of our derived distances is about a factor of 5 better than the case without the color calibrations. The current result supports our previous assertion (Paper II) that the largest source of isochrone mismatches stems mainly from the conversion of theoretically predicted quantities into magnitudes and colors.

We also found that our photometric metallicities are in good agreement with spectroscopic estimates although the largest difference was found for Praesepe, where the photometric metallicity was $\sim 2 \sigma$ higher than the spectroscopic value. In addition, we found an excellent agreement for the Pleiades between our derived distance and the geometric determinations other than the original Hipparcos measurements, confirming the validity of the empirically calibrated isochrones.

We explored various systematic errors in distance, photometric metallicity, and reddening estimates. The distance from MS fitting is sensitive to the metallicity and helium abundance but can be estimated to an accuracy of $\pm 1 \%$ (fitting error) and $\pm 2 \%$ (systematic error) if there exists a good spectroscopic abundance measurement. When we determine cluster metallicity and/or reddening with our isochrones alone, the distance becomes sensitive to the size of the systematic zero-point errors in the photometry. With reasonable sizes of the zero-point errors, however, distances can be determined with comparable accuracy to those at the spectroscopic metallicities.

We showed that the Pleiades $\mathrm{K}$ dwarfs are bluer than the calibrated isochrone in $(B-V, V)$, confirming the previous study by Stauffer et al. (2003). We further showed that the red stars in NGC 2516 are bluer than the isochrone when the S02 data are used; however, the JTH01 data closely match the iso- chrone down to the limit of the isochrone calibration. Using a limited set of $v \sin i$ data, we found that a few stars with high $v \sin i$ are fainter by $0.1-0.2 \mathrm{mag}$ than the slow rotators in $0.8 \leq(B-V)_{0} \leq 1.0$. This suggests that the K dwarfs in NGC 2516 may also exhibit the blue K dwarf phenomenon, although the degree of departure is smaller than in the Pleiades. More $v \sin i$ data for lower mass stars and careful photometric standardization are required to set the upper age limit of the anomalous colors of active $\mathrm{K}$ dwarfs. For young open clusters, careful attention should be given to this kind of anomaly to avoid erroneous results in distance.

We also derived a helium abundance for the Pleiades of $Y=0.279 \pm 0.015$, based on the average geometric distance to the Pleiades (Table 1). This value is equal within the errors to the Sun's initial helium abundance and that of the Hyades. This result implies that there is no observed cosmic scatter in the helium abundance at the level of the accuracy in the current estimation. To decrease the size of the error, more accurate abundances for the cluster and smaller systematic errors in the photometry are required.

There are distinct limits to the current calibration. The dependence of the MS luminosity and colors on metallicity cannot be inferred from one calibrating cluster alone and must be either assumed to be correct in the models (as in this paper) or else checked against local field stars with known distances and metallicity (D. M. Terndrup et al. 2007, in preparation). The Hipparcos data in the Hyades are only complete to $V \approx 11\left(M_{V} \approx 8\right)$, so the empirical calibration in Paper II extends only down to $\sim 0.7 M_{\odot}$. In addition, the Hyades has an age of about $550 \mathrm{Myr}$, so younger clusters should be used to calibrate the upper MS for stars more massive than the Hyades turnoff stars. All of these issues will be addressed with photometry of low-mass members in Praesepe and $\alpha$ Persei in the later papers of this series.

We are greatly indebted to Jennifer Johnson for her detailed and thoughtful reading of the first draft of this paper. Many suggestions by Constantine Deliyannis are gratefully acknowledged. We also thank Andrew Gould for useful discussions on the proper motion of cluster members and Hwankyung Sung for detailed description on his photometry. The work reported here was partially supported by grants AST 02-06008 and AST 02-05789 from the National Science Foundation to the Ohio State Research Foundation and with funds provided by the Ohio State University Department of Astronomy. D. B. P. acknowledges support from the NASA Postdoctoral Program, which is administered by the Oak Ridge Associated Universities. This publication makes use of data products from the Two Micron All Sky Survey, which is a joint project of the University of Massachusetts and the Infrared Processing and Analysis Center/California Institute of Technology, funded by the National Aeronautics and Space Administration and the National Science Foundation.

\section{APPENDIX}

\section{THE HYADES PHOTOMETRIC CALIBRATION REVISITED}

In $\S 6.1$ we estimated the systematic errors in distance, photometric metallicity, and reddening that would result from zero-point errors in the photometry of target clusters. Here we elaborate on the systematic errors in the Hyades photometry, which determine the accuracy of the calibration itself.

In our assembly of the Hyades photometry in Paper II, we employed the Bessell (1979) transformation between the Johnson and Cousins $V-I$ systems, namely,

$$
(V-I)_{\mathrm{C}}=0.778(V-I)_{\mathrm{J}}
$$


TABLE 15

Comparison of Johnson and Kron Transformations

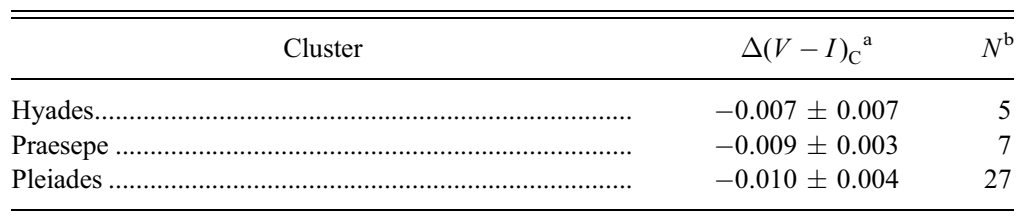

a Transformed Johnson minus Kron photometry from previous transformations employed in Paper II (see text).

$\mathrm{b}$ Number of stars used in the comparison.

This calibration has a small zero-point error, in that this produces colors that are somewhat too blue. Cousins (1980), for example, derived

$$
(V-I)_{\mathrm{C}}=0.77(V-I)_{\mathrm{J}}+0.01 .
$$

As part of a detailed study of local field stars, we have assembled a large sample of stars with both Cousins and Johnson photometry (D. M. Terndrup et al. 2007, in preparation). These indicate

$$
(V-I)_{\mathrm{C}}=0.779(V-I)_{\mathrm{J}}+0.014 .
$$

Although the size of the zero point is small, it produces a systematic error in distance of $\sim 0.1$ mag. This is because the Johnson data for the Hyades mostly have $M_{V} \leq 6$ where the average slope of the MS is $\Delta V / \Delta(V-I)_{\mathrm{C}} \approx 7$.

As summarized in Table 15, there are a few stars in the Hyades, Praesepe, and the Pleiades that have $V-I$ photometry on both the Johnson and Kron systems. These provide additional evidence that a small redward shift in the transformation of the Johnson colors is needed. The second column shows the mean magnitude difference in the sense of Johnson minus Kron after the former were placed on the Cousins system using the (incorrect) transformation in equation (A1). The Kron colors were transformed to the Cousins system using the cubic polynomial derived by Bessell \& Weis (1987). Errors are the standard error of the mean difference, and the number of stars used in the comparison is shown in the last column. In all cases, the Johnson-to-Cousins transformation from equation (A1) employed in Paper II produces colors that are slightly too blue compared to those from the Kron system.

The adoption of the new Johnson-to-Cousins transformation (eq. [A3]) modifies our Hyades calibration, making the Hyades empirical isochrone from Paper II slightly redder in the upper MS since the photometry for these stars is mainly on the Johnson system. The net effect is to make our color- $T_{\text {eff }}$ correction table (Table 2 in Paper II) redder by $\Delta(V-I)_{\mathrm{C}}=0.012$ for $M_{V} \leq 6$. The isochrones used in this paper included this correction, no adjustment for $M_{V} \geq 8$, and a linear transition between these two magnitude limits. We also employed equation (A3) in the assembly of the Praesepe and the Pleiades photometry in this paper.

In Paper II, we did not include $V-I_{\mathrm{C}}$ from Taylor \& Joner (1985) because we were unable to achieve a good transformation between their photometry and those on the Johnson system from other sources (Johnson et al. 1966, 1968; Mendoza 1967). The exclusion of their data prompted Taylor \& Joner (2005) to discuss their photometry at length and to examine whether there were systematic errors in the source photometry used in our previous work. They first verified that their $V-I_{\mathrm{C}}$ values are about $0.02 \mathrm{mag}$ redder than ours. Then, they identified two sources of the difference: the adjustment in the Johnson-Cousins transformation noted above (but derived independently here) and a systematic error in Mendoza's $R-I_{\mathrm{J}}$ photometry.

Recently, Joner et al. (2006) published new Cousins photometry of a large sample of Hyades stars. Their data set has the advantage over the previous collection in that it was taken on a single telescope and filter set. In Figure 23 we plot the differences in the photometry for the stars used to calibrate the Hyades isochrone (Paper II) and the data in Joner et al. (2006). Compared to our values in Paper II, their photometry is brighter in $V$ and slightly redder in $B-V$. In $V-I_{\mathrm{C}}$, the bright stars in our sample are bluer than the Joner et al. (2006) values, but both data are in agreement for the fainter stars. The mean differences and standard errors for the range used in the MS fitting of this paper $\left(M_{V}>3.1\right)$ are $\Delta V=+0.026 \pm 0.002, \Delta(B-V)=-0.009 \pm 0.001$, and $\Delta(V-I)_{\mathrm{C}}=+0.004 \pm 0.002$.

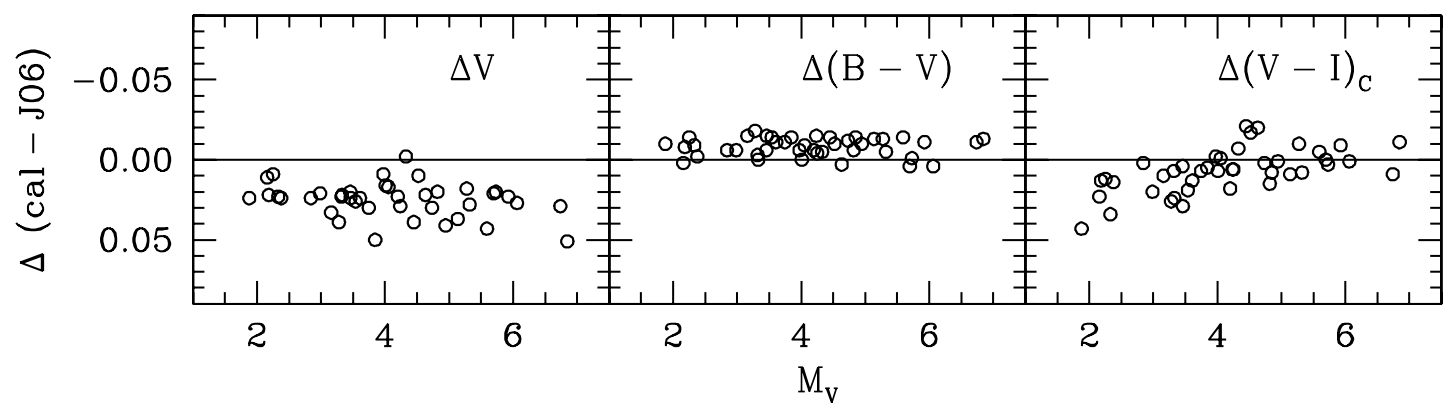

FIG. 23.-Differences between the magnitudes and colors of the Hyades members used to calibrate the Hyades empirical isochrone (Paper II) and photometry of Joner

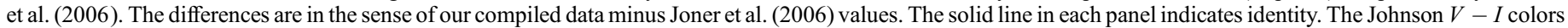
used in Paper II were transformed to the Cousins system using an updated transformation (see text). 
These differences can be compared to those found previously (Table 2) between the Tycho photometry and our values in Paper II. Compared to the Tycho-1, our Hyades values are also fainter by 0.012 mag and are bluer in $B-V$ by 0.009 mag. If we had adjusted zero points in $V, B-V$, and $V-I_{\mathrm{C}}$ of isochrones to the Joner et al. (2006) scales, the distances at spectroscopic metallicities would become longer than our estimated values in Table 7 by $0.01 \mathrm{mag}$ for Praesepe, 0.03 mag for the Pleiades, and 0.02 mag for both M67 and NGC 2516.

Abt, H. A., \& Levy, S. G. 1972, ApJ, 172, 355

Alonso, A., Arribas, S., \& Martínez-Roger, C. 1995, A\&A, 297, 197 1996, A\&AS, 117, 227

Bahcall, J. N., \& Pinsonneault, M. H. 2004, Phys. Rev. Lett., 92, 121301

Bahcall, J. N., Pinsonneault, M. H., \& Basu, S. 2001, ApJ, 555, 990

Barrado y Navascués, D., Stauffer, J. R., Bouvier, J., \& Martín, E. L. 2001, ApJ, 546, 1006

Basu, S., Pinsonneault, M. H., \& Bahcall, J. N. 2000, ApJ, 529, 1084

Belikov, A. N., Hirte, S., Meusinger, H., Piskunov, A. E., \& Schilbach, E. 1998, A\&A, 332, 575

Bernstein, R., Shectman, S. A., Gunnels, S. M., Mochnacki, S., \& Athey, A. E. 2003, Proc. SPIE, 4841, 1694

Bessell, M. S. 1979, PASP, 91, 589 2005, ARA\&A, 43, 293

Bessell, M. S., Castelli, F., \& Plez, B. 1998, A\&A, 333, 231

Bessell, M. S., \& Weis, E. W. 1987, PASP, 99, 642

Boesgaard, A. M. 1989, ApJ, 336, 798

Boesgaard, A. M., \& Friel, E. D. 1990, ApJ, 351, 467

Bouvier, J., Duchêne, G., Mermilliod, J.-C., \& Simon, T. 2001, A\&A, 375, 989

Bouvier, J., Rigaut, F., \& Nadeau, D. 1997, A\&A, 323, 139

Breger, M. 1986, ApJ, 309, 311

Burke, C. J., Pinsonneault, M. H., \& Sills, A. 2004, ApJ, 604, 272

Burstein, D., Faber, S. M., \& Gonzalez, J. J. 1986, AJ, 91, 1130

Burstein, D., \& Heiles, C. 1982, AJ, 87, 1165

Cardelli, J. A., Clayton, G. C., \& Mathis, J. S. 1989, ApJ, 345, 245

Carpenter, J. M. 2001, AJ, 121, 2851

Castelaz, M. W., Persinger, T., Stein, J. W., Prosser, J., \& Powell, H. D. 1991, AJ, 102, 2103

Castelli, F., Gratton, R. G., \& Kurucz, R. L. 1997, A\&A, 318, 841

Cayrel, R., Cayrel de Strobel, G., \& Campbell, B. 1988, in IAU Symp. 132, The Impact of Very High S/N Spectroscopy on Stellar Physics, ed. G. Cayrel de Strobel \& M. Spite (Dordrecht: Kluwer), 449

Cousins, A. W. J. 1980, S. African Astron. Obs. Circ., 1, 234

Cousins, A. W. J., \& Caldwell, J. A. R. 1985, Observatory, 105, 134

Dachs, J., \& Kabus, H. 1989, A\&AS, 78, 25

Damiani, F., Flaccomio, E., Micela, G., Sciortino, S., Harnden, F. R., Jr., Murray, S. S., Wolk, S. J., \& Jeffries, R. D. 2003, ApJ, 588, 1009

Dean, J. F., Warren, P. R., \& Cousins, A. W. J. 1978, MNRAS, 183, 569

de Bruijne, J. H. J., Hoogerwerf, R., \& de Zeeuw, P. T. 2001, A\&A, 367, 111

Dickens, R. J., Kraft, R. P., \& Krzeminski, W. 1968, AJ, 73, 6

Duquennoy, A., \& Mayor, M. 1991, A\&A, 248, 485

Eggen, O. J. 1950, ApJ, 111, 81

1972, ApJ, 173, 63

1981, ApJ, 247, 503

1983, MNRAS, 204, 377

Feinstein, A., Marraco, H. G., \& Mirabel, I. 1973, A\&AS, 9, 233

Friel, E. D., \& Boesgaard, A. M. 1992, ApJ, 387, 170

Garcia Lopez, R. J., Rebolo, R., \& Beckman, J. E. 1988, PASP, 100, 1489

Gatewood, G., \& de Jonge, J. K. 1994, ApJ, 428, 166

Gatewood, G., de Jonge, J. K., \& Han, I. 2000, ApJ, 533, 938

González, J. F., \& Lapasset, E. 2000, AJ, 119, 2296

Green, E. M. 1988, in Calibration of Stellar Ages, ed. A. G. D. Phillip (Schenectady: Davis), 81

Grevesse, N., \& Sauval, A. J. 1998, Space Sci. Rev., 85, 161

Halbwachs, J. L., Mayor, M., Udry, S., \& Arenou, F. 2003, A\&A, 397, 159

Hobbs, L. M., \& Thorburn, J. A. 1991, AJ, 102, 1070

Høg, E., et al. 2000, A\&A, 355, L27

Iriarte, B. 1969, Bol. Obs. Tonantzintla y Tacubaya, 5, 89

Janes, K. A., \& Smith, G. H. 1984, AJ, 89, 487

Jeffries, R. D., Thurston, M. R., \& Hambly, N. C. 2001, A\&A, 375, 863 (JTH01)

Johns-Krull, C. M., \& Anderson, J. 2005, in Proc. 13th Cool Stars Workshop, Cool Stars, Stellar Systems, and the Sun, ed. F. Favata, G. Hussain, \& B. Battrick (Noordwijk: ESA), 683

Johnson, H. L. 1952, ApJ, 116, 640 1957, ApJ, 126, 121

Johnson, H. L., Iriarte, B., Mitchell, R. I., \& Wisniewski, W. Z. 1966, Comm. Lunar Planet. Lab., 4, 99

Johnson, H. L., MacArthur, J. W., \& Mitchell, R. I. 1968, ApJ, 152, 465
REFERENCES

Joner, M. D., Taylor, B. J., Laney, C. D., \& van Wyk, F. 2006, AJ, 132, 111

Jones, B. F. 1972, ApJ, 171, L57 1981, AJ, 86, 290

King, J. R., Soderblom, D. R., Fischer, D., \& Jones, B. F. 2000, ApJ, 533, 944 Klein Wassink, W. J. 1927, Publ. Kapteyn Astron. Lab. Groningen, 41, 1

Kroupa, P., Tout, C. A., \& Gilmore, G. 1993, MNRAS, 262, 545

Landolt, A. U. 1979, ApJ, 231, 468

Lejeune, Th., Cuisinier, F., \& Buser, R. 1997, A\&AS, 125, 229 1998, A\&AS, 130, 65

Loktin, A. V. 2000, Astron. Lett., 26, 657

Makarov, V. V. 2002, AJ, 124, 3299 2003, AJ, 126, 2408

Mamajek, E. E., Meyer, M. R., \& Liebert, J. 2002, AJ, 124, 1670

Mathis, J. S. 1990, ARA\&A, 28, 37

Mendoza, E. E. 1967, Bol. Obs. Tonantzintla y Tacubaya, 4, 149

Mermilliod, J.-C. 1981a, A\&A, 97, 235 1981b, A\&AS, 44, 467

Mermilliod, J.-C., \& Mayor, M. 1999, A\&A, 352, 479

Mermilliod, J.-C., \& Paunzen, E. 2003, A\&A, 410, 511

Mermilliod, J.-C., Rosvick, J. M., Duquennoy, A., \& Mayor, M. 1992, A\&A, 265,513

Mermilliod, J.-C., Turon, C., Robichon, N., Arenou, F., \& Lebreton, Y. 1997, in Hipparcos-Venice '97, ed. B. Battrick \& M. A. C. Perryman (ESA SP-402; Paris: ESA), 643

Mermilliod, J.-C., Weis, E. W., Duquennoy, A., \& Mayor, M. 1990, A\&A, 235, 114

Meynet, G., Mermilliod, J.-C., \& Maeder, A. 1993, A\&AS, 98, 477

Mitchell, R. I., \& Johnson, H. L. 1957, ApJ, 125, 414

Montgomery, K. A., Marschall, L. A., \& Janes, K. A. 1993, AJ, 106, 181 (MMJ93)

Moraux, E., Bouvier, J., \& Stauffer, J. R. 2001, A\&A, 367, 211

Munari, U., Dallaporta, S., Siviero, A., Soubiran, C., Fiorucci, M., \& Girard, P. 2004, A\&A, 418, L31

Narayanan, V. K., \& Gould, A. 1999a, ApJ, 515, 256 1999b, ApJ, 523, 328

Nicolet, B. 1981, A\&A, 104, 185

Nissen, P. E. 1988, A\&A, 199, 146

Nissen, P. E., Twarog, B. A., \& Crawford, D. L. 1987, AJ, 93, 634

Oja, T., \& Evans, D. W. 1998, A\&A, 333, 673

Pan, X., Shao, M., \& Kulkarni, S. R. 2004, Nature, 427, 326

Patience, J., Ghez, A. M., Reid, I. N., \& Matthews, K. 2002, AJ, 123, 1570

Paulson, D. B., Sneden, C., \& Cochran, W. D. 2003, AJ, 125, 3185

Percival, S. M., Salaris, M., \& Groenewegen, M. A. T. 2005, A\&A, 429, 887

Percival, S. M., Salaris, M., \& Kilkenny, D. 2003, A\&A, 400, 541

Perryman, M. A. C., et al. 1997a, A\&A, 323, L49

. 1997b, The Hipparcos and Tycho Catalogues (ESA SP-1200;

Noordwijk: ESA)

- 1998, A\&A, 331, 81 2001, A\&A, 369, 339

Pinsonneault, M. H., \& Stanek, K. Z. 2006, ApJ, 639, L67

Pinsonneault, M. H., Stauffer, J. R., Soderblom, D. R., King, J. R., \& Hanson,

R. B. 1998, ApJ, 504, 170

Pinsonneault, M. H., Terndrup, D. M., Hanson, R. B., \& Stauffer, J. R. 2003, ApJ, 598, 588 (Paper I) 2004, ApJ, 600, 946 (Paper II)

Pinsonneault, M. H., Terndrup, D. M., \& Yuan, Y. 2000, in ASP Conf. Ser. 198, Stellar Clusters and Associations: Convection, Rotation, and Dynamics, ed. R. Pallavicini, G. Micela, \& S. Sciortino (San Francisco: ASP), 95

Press, W. H., Teukolsky, S. A., Vetterling, W. T., \& Flannery, B. P. 1992, Numerical Recipes in C (2nd ed.; Cambridge: Cambridge Univ. Press)

Prosser, C. F., Stauffer, J. R., \& Kraft, R. P. 1991, AJ, 101, 1361

Randich, S., Sestito, P., Primas, F., Pallavicini, R., \& Pasquini, L. 2006, A\&A, 450,557

Reid, I. N. 1999, ARA\&A, 37, 191

Robichon, N., Arenou, F., Mermilliod, J.-C., \& Turon, C. 1999, A\&A, 345, 471

Rousseeuw, P. J. 1990, in Handbook of Statistical Methods, ed. H. M. Wadsworth (New York: McGraw-Hill), 16

Salpeter, E. E. 1955, ApJ, 121, 161

Sandquist, E. L. 2004, MNRAS, 347, 101 (S04) 
Schilbach, E., Robichon, N., Souchay, J., \& Guibert, J. 1995, A\&A, 299, 696

Schlegel, D. J., Finkbeiner, D. P., \& Davis, M. 1998, ApJ, 500, 525

Scholz, A., \& Eislöffel, J. 2004, A\&A, 419, 249

Schultz, G. V., \& Wiemer, W. 1975, A\&A, 43, 133

Siegel, M. H., Majewski, S. R., Reid, I. N., \& Thompson, I. B. 2002, ApJ, 578, 151

Sills, A., Pinsonneault, M. H., \& Terndrup, D. M. 2000, ApJ, 534, 335

Sneden, C., Gehrz, R. D., Hackwell, J. A., York, D. G., \& Snow, T. P. 1978, ApJ, 223, 168

Sneden, C. A. 1973, Ph.D. thesis, Univ. Texas

Snowden, M. S. 1975, PASP, 87, 721

Soderblom, D. R., King, J. R., Hanson, R. B., Jones, B. F., Fischer, D., Stauffer, J. R., \& Pinsonneault, M. H. 1998, ApJ, 504, 192

Soderblom, D. R., Nelan, E., Benedict, G. F., McArthur, B., Ramirez, I., Spiesman, W., \& Jones, B. F. 2005, AJ, 129, 1616

Soderblom, D. R., Stauffer, J. R., Hudon, J. D., \& Jones, B. F. 1993, ApJS, 85, 315

Southworth, J., Maxted, P. F. L., \& Smalley, B. 2005, A\&A, 429, 645

Stauffer, J. R. 1982a, AJ, 87, 899 $1982 \mathrm{~b}, \mathrm{AJ}, 87,1507$

1982c, PASP, 94, 678

1984, ApJ, 280, 189

2001, in ASP Conf. Ser. 244, Young Stars Near Earth: Progress and

Prospects, ed. R. Jayawardhana \& T. Greene (San Francisco: ASP), 127

Stauffer, J. R., Hamilton, D., \& Probst, R. G. 1994, AJ, 108, 155

Stauffer, J. R., Hamilton, D., Probst, R., Rieke, G., \& Mateo, M. 1989, ApJ, 344, L21

Stauffer, J. R., \& Hartmann, L. W. 1987, ApJ, 318, 337

Stauffer, J. R., Jones, B. F., Backman, D., Hartmann, L. W., Barrado y Navascués, D., Pinsonneault, M. H., Terndrup, D. M., \& Muench, A. A. 2003, AJ, 126, 833
Stauffer, J. R., Klemola, A., Prosser, C., \& Probst, R. 1991, AJ, 101, 980 Stauffer, J. R., Schultz, G., \& Kirkpatrick, J. D. 1998, ApJ, 499, L199 Stello, D., \& Nissen, P. E. 2001, A\&A, 374, 105

Stetson, P. B., Bruntt, H., \& Grundahl, F. 2003, PASP, 115, 413

Sung, H., Bessell, M. S., Lee, B.-W., \& Lee, S.-G. 2002, AJ, 123, 290 (S02)

Tautvaišienè, G., Edvardsson, B., Tuominen, I., \& Ilyin, I. 2000, A\&A, 360, 499

Taylor, B. J. 1980, AJ, 85, 242

Taylor, B. J., \& Joner, M. D. 1985, AJ, 90, 479 2005, ApJS, 159, 100

Terndrup, D. M., Pinsonneault, M. H., Jeffries, R. D., Ford, A., Stauffer, J. R., \& Sills, A. 2002, ApJ, 576, 950

Torres, G., \& Ribas, I. 2002, ApJ, 567, 1140

Upgren, A. R., Weis, E. W., \& DeLuca, E. E. 1979, AJ, 84, 1586

VandenBerg, D. A., \& Clem, J. L. 2003, AJ, 126, 778

van Leeuwen, F. 1999, A\&A, 341, L71

$$
\text { 2005, A\&A, 439, } 805
$$

van Leeuwen, F., Alphenaar, P., \& Meys, J. J. M. 1987, A\&AS, 67, 483

van Leeuwen, F., \& Evans, D. W. 1998, A\&AS, 130, 157

van Leeuwen, F., Evans, D. W., Grenon, M., Grossmann, V., Mignard, F., \&

Perryman, M. A. C. 1997, A\&A, 323, L61

van Leeuwen, F., \& Fantino, E. 2005, A\&A, 439, 791

Verschoor, J. N., \& van Genderen, A. M. 1983, A\&AS, 53, 419

Wegner, W. 2003, Astron. Nachr., 324, 219

Weis, E. W. 1981, PASP, 93, 437

Yong, D., Carney, B. W., \& de Almeida, M. L. T. 2005, AJ, 130, 597

Zwahlen, N., North, P., Debernardi, Y., Eyer, L., Galland, F., Groenewegen, M. A. T., \& Hummel, C. A. 2004, A\&A, 425, L45 